\title{
Street ARt
}

Using Augmented Reality to Create Digital Street Art

Stefan Peacock 



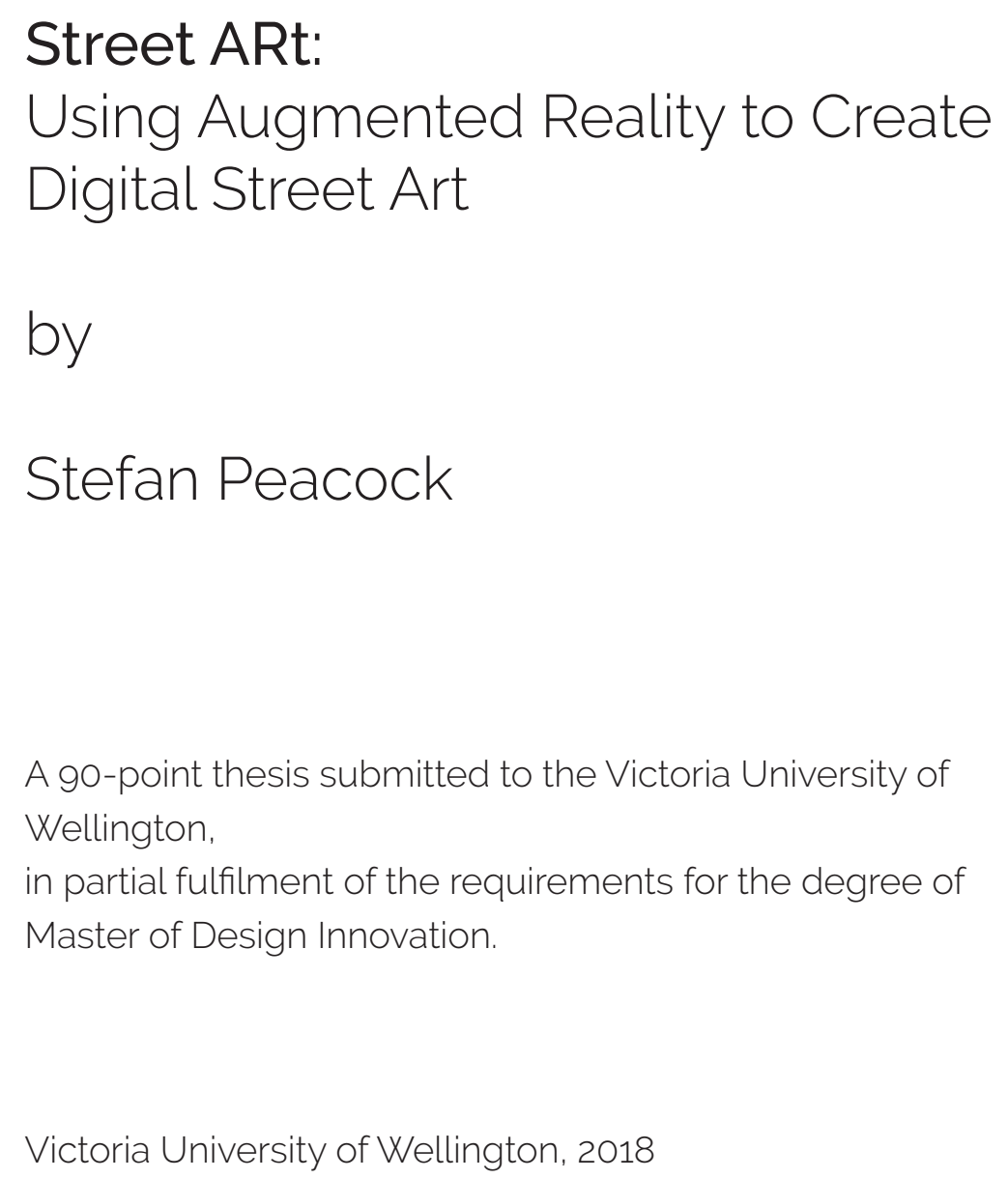




\section{Foreword}


After assisting in research focused on the use of new technologies for gallery and institutional spaces, I felt a desire to explore these technologies in a way that could be available to everyone. This led to a broad interest in using Augmented Reality and other new technologies to make art more accessible to the public. This thesis and its topic area is an extension of this desire 
Acknowledgements 
My Supervisor Leon Gurevitch: Thank you for your supervision throughout this thesis, in particular I appreciate your unwavering patience, understanding and support, while still providing the blunt feedback and push that I needed.

My secondary supervisor Byron Mallett: Thank you for all your support, as well as your positivity and creative energy. Our discussions allowed for the exploration of topics I would never have considered.

Catherine Caudwell for coordinating the MDI program and always being available to assist with feedback and insight.

Stefan Schultz, Phoebe Zeller, Sophie Price and Ivy Calvert: Thank you for always being there when I needed you, whether it was for bouncing ideas around, feedback or just a kind word. I appreciate you being there while we struggled through.

Jonathon Bishop: Thank you for contributing to my original interest in the topic area, and giving me many opportunities in the area of AR.
The Simon, Cathy and Jackie Peacock: Thank you for encouraging my creativity and allowing me to explore that from an early age.

Sian Killick and Siobhan Peacock: thanks for making sure I got through this, and supporting me no matter what

MDI students of 2018: Thanks for being there with suggestions, feedback and laughs. Your comradery was always appreciated.

Joseph Hendren: Thank you for proofreading my thesis and accompanying me on my explorations of the street.

Alexander Saunders: Thanks for assisting with the documentation of my designs. 
Abstract 
The long-imagined fiction of a digitally supplemented world is fast becoming a reality. Augmented Reality technology is advancing at a rapid rate, approaching mass adoption and use. Available to anyone with a modern mobile phone, Augmented Reality allows for a number of possibilities, augmenting the physical world with information or content of varying types. These possibilities have a number of implications for both utility and creative expression. This thesis explores the use of Augmented Reality as a tool for creative expression, comparing its use of the physical world to Street Art's use of the street. Street Art uses the street as an artistic resource (Riggle, 2010) to provoke and elicit response. Augmented Reality uses physical location and context to strengthen its content and deliver information or entertainment. Augmented Reality Artworks have contested physical space (Skwarek, 2014; Veenhof \& Skwarek, 2010) and shifted the boundaries of curation (Garbe, 2014).
This thesis compares the histories of Augmented Reality and Street Art, resulting in a proposal for using Augmented Reality as a method for Street Art, allowing artists to create Digital Street Art. Research through Design explores this practice, creating Digital Street Art works that use the possibilities of Augmented Reality technology to use the street as an artistic resource. Using digital techniques like animation, interactivity, data visualisation and three-dimensional imagery; this thesis aims to explore how the Digital Street Artist can create work that engages with the public space and provoke a response. This is explored through several Digital Street Art responses to the public space and issues that can be addressed within it. These responses are designed to use digital techniques and AR technology to make artistic use of the street. Allowing it to be both Digital in nature and Street Art in essence. 


\section{Contents}




\begin{tabular}{|l} 
Foreword \\
Acknowledgements \\
Abstract \\
Contents
\end{tabular}

Location and Content Exploration ..............................36

Findings .................................................. 50

Chapter 4: Digital Street Art ............................52

Cats and Mice ............................................... 54

Disposable Consequences ............................................ 72

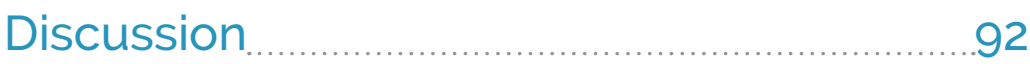

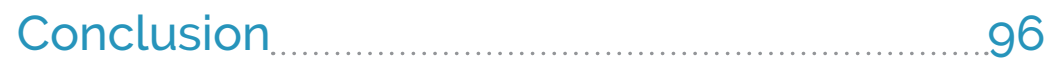

List of Figures ................................................ 100

References ............................................. 102 
Introduction 
For years science fiction has imagined worlds supplemented and dominated by digital content. Today, that fiction is becoming a very real eventuality. Augmented Reality (AR) is a technology that is approaching a large scale of adoption and acceptance, not only known to developers and researchers, but to anyone paying attention to the news. This is hardly surprising given the possibilities that Augmented Reality can bring. By adding digital entities to a physical world (Reitmayr \& Schmalstieg, 2003), Augmented Reality can supplement information, add interesting and engaging digital content to physical objects and create complex digital entities. These possibilities allow for a number of interesting use cases with the technology, both as a practical tool, and as a means of creative expression.

Street Art has long been disregarded as the work of thugs, vandals and criminals (Dickinson, 2008). However, with the rising popularity of Street Artists like Banksy ('Banksy', n.d.), Shepard Fairey ('Obey Giant - The Art of Shepard Fairey', 2018) and Invader (Invader, n.d.), there is a shift occurring in the perception and acceptance of Street Art. This shift has resulted in galleries and private collectors willing to pay large amounts to own artworks from popular artists. Despite this attitude, the street is where street art belongs - necessarily using the street as an artistic resource integral to fully appreciating it's meaning (Riggle, 2010). Once it removed Street Art loses its meaning, essence and authenticity. Using the public nature of the street allows Street Art to engage with the public, outside the bounds of the artworld. Challenging and confronting the world to provoke responses.
By fully investigating the context and histories of both Augmented Reality and Street Art, this thesis addresses how Augmented Reality can be used as a new method for Street Art; creating Digital Street Art. The possibilities of Augmented Reality allow digital content to be created that can draw on the physical environment. Digital Street Art then allows for the use of features like animation, three-dimensional imagery, interactivity and data. This allows the artist to create Street Art that engages with the public and potentially elicit a response using these techniques. This informs the following research question:

How can Augmented Reality be used to create Digital Street Art that engages with the public space and provokes a response?

Using the city of Wellington as an example, this thesis uses research through design to create works of Digital Street Art. These works seek to engage with the public and provoke a response through the use of Augmented Reality. 


\section{Chapter $1:$ \\ Methodologies}


This thesis begins with an establishment of context. The histories of Augmented Reality research and Street Art research are addressed and discussed through a literature review that leads into the proposed practice of Digital Street Art. This allows for the use of digital techniques in a physical location through Augmented Reality technology.

The practice of Digital Street Art is explored through the overarching method of research through design (Frayling, 1993). Cristopher Frayling's paper Research Through Art and Design is often cited and details research through design alongside two other methodologies; research into design and research for design. Research into design is research into either the history of design, aesthetics of design, or research into theoretical perspective on design. Research for design aims to conduct research so as to create a designed output. This output is designed to stand on its own, with the research serving only as precedent or reference.

For the purposes of this thesis I have taken an "action - reflection approach" to research through design (Jonas, 2007). Action research seeks to investigate new ideas by testing them within the real world and communicating this knowledge (Archer, 1995). Reflective practice is complementary to this, outlining a problem and working towards a solution. Constantly reflecting on the actions taken to address this problem results in a "reflective conversation with the situation" (Valkenburg \& Dorst, 1998 p. 251). The combined action-reflection approach is focused on creating design knowledge rather than a design solution. The research is not restricted to the product or service being designed, but rather aimed at discussing the explanations and ideas related to the context of the designed output. This method is growing rapidly, and is used to great effect in design enquiry. (Frankel \& Racine, 2010).

This thesis uses the action - reflection approach throughout Chapters 3 and 4, which was focused on the creation of Digital Street Art. Free experiments investigated technology, precedent and themes before reflecting on these to inform further development. Chapter 4 then engages with this process further, designing Digital Street Art responses to scenarios driven by public issues. These works are designed to engage with the public and provoke a response. 
Chapter 2:

Context of Research 
This research has been situated between the areas of Augmented

Reality and Street Art. To properly combine and compare these areas

it is important to address them fully, understanding their definitions,

histories and research. A literature review into these fields was conducted,

identifying parallels and contrasts to formulate the context of this research.

Augmented reality as a technology has been researched for several decades, accelerating in recent years due to an increase in popularity and availability. This has brought more research into content and uses for the technology, supplementing the initial research into functionality. Street Art in a similar manner is growing in popularity and acceptance, influencing the amount of academic knowledge in the area, as well as the popularity and prevalence of the medium. 


\section{Augmented Reality}

Ivan Sutherland's (1968) The Sword of Damocles is widely considered to be the first AR system. This system was a head-mounted, see-through display that projected simple wireframe graphics into the user's vision. The research was mostly focused on trying to develop a head mounted stereo display, focusing on the user's reactions and use of the display. This was a very early and simple precursor to the head-mounted displays available today. Compared to these modern options, The Sword of Damocles was large, weighty and unwieldy, its name stemming from the need for a support bar attached to the ceiling to support the user's head.

In the 1970s and 80s Myron Kreuger's Videoplace experiments used a different technical approach to Augmented Reality, using projectors and video cameras to place users in a digital graphic environment within a physical room. This allowed for users to feel immersed in a graphical space and interact with the graphics they could see projected. (Krueger, Gionfriddo, \& Hinrichsen, 1985).

In the 1990s, Augmented Reality research and development began in earnest, with several defining papers being penned. The term "Augmented Reality" was first used by Boeing researcher Tom Caudell in 1992

(Caudell \& Mizell, 1992). Other noticeable research included Augmented Reality: A class of displays on the reality-virtuality continuum (Milgram, Takemura, Utsumi, \& Kishino, 1995), a paper still frequently cited and discussed. The research during this period defined the majority of in prevalence and popularity, moving from research labs into the general public's eye. While hardware for high fidelity AR systems is still being developed and researched, the main cause for this popularity is the arrival of AR on smart phones. Good quality cameras and smaller, powerful CPU's have resulted in smart phones being able to run AR systems and experiences. The result has been accessibility for the casual user, able to use $\mathrm{AR}$ on the device they already carry and use in public.

Commercially made and distributed Augmented Reality applications have only helped the mobile Augmented Reality field to grow, resulting in smartphone manufacturers and mobile operating systems offering built-in support for Augmented Reality (Apple Inc., 2018a; Google LLC, n.d.) . This has encouraged more developers to experiment with the technology to create more Augmented Reality applications. This has led to an increase in AR functionality within mobile applications downloaded (M. Campbell, 2018). This clearly show an increase in the popularity and acceptance of the technology, but what does Augmented Reality actually entail?

\section{Defining Augmented Reality}

Augmented Reality (AR) has always been a constantly advancing and shifting field. New research develops new technology, leading to a shift and contrast in the definition of the term. Some early definitions focused on comparing AR to virtual reality, while others aimed at broadening the field and the technology. 
Paul Milgram’s (1995) Augmented Reality: A class of displays on the reality-virtuality continuum discussed this, opening with an acknowledgement of the lack of consistent definition despite its increasing use. The paper continues to describe two definitions commonly used: "augmenting natural feedback to the operator with simulated cues" and "a form of virtual reality where the participant's head-mounted display is transparent, allowing a clear view of the world"(Milgram et al., 1995 p. 283). The difference in these two definitions lies in their breadth. Each of these terms describe Augmented Reality to some extent, however one clearly limits the definition to a specific type of hardware and method, while the other does not. Two main types of AR displays are discussed and compared to these definitions. See-through displays augment the viewer's vision by projecting digital content onto some form of display through which the user can still see the physical environment. Video-based displays place digital content onto a live video feed that the user can see (Milgram et al., 1995). These are both valid types of AR that are still used and researched today, showing that a narrow definition of the term does not serve the field or the research conducted.

Milgram continues to place Augmented Reality on a Reality-Virtuality Continuum, which defines a scale from the fully real world to a fully virtual world (Milgram et al, 1995). More specifically it exists within the field of Mixed Reality, which encompasses all technology that blends or compares digital content with the real world. It allows both real world objects and digital objects, or information, to be seen with the same display (Tamura et al, 2001). Mixed Reality also encompasses Augmented
Virtuality, which can be seen as the opposite of Augmented Reality. This takes a fully virtual world and adds real world data, information or visuals to it. For example, this could be a virtual game environment being affected by real world location or events. Or an experience affected by other users or observers interacting with it (Milgram et al., 1995).

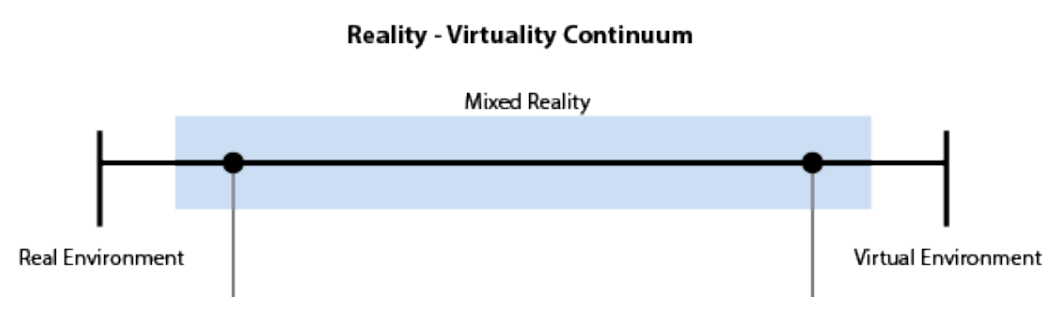

Figure 1: Milgram's Reality-Virtuality Continuum, adapted from Milgram et al. (1995)

Ronald Azuma's (1997) definition followed Milgram's research, and is a definition familiar within AR research. This defined it as a variation of virtual reality that allowed the user to see the real world supplemented with virtual objects. Therefor Augmented Reality should combine the real and virtual, be interactive in real time and be registered in three dimensions. (R. T. Azuma, 1997). This definition evolved three years later, with the same author defining it as a system that "combines real and virtual objects in a real environment; runs interactively, and in real time; and registers (aligns) real and virtual objects with each other"

(R. Azuma et al., 2001). This demonstrates how the definition continues to shift and become more concrete and encompassing as the field is developed. This definition, used in several research papers, is persuasive, however it focuses solely on visual augmentation. 
Another accepted definition, that is broader in scope, includes "user interface technology that augments the users' (physical) environment with computer generated entities" (Reitmayr \& Schmalstieg, 2003 p. 65). As well as including non-visual augmentation, this definition also serves in removing the dichotomy of "real" and "virtual". Digital entities are still real and exist within the perception of the viewer, the only difference is that they are not physically made.

All of these definitions describe Augmented Reality, but the broader definitions encompass more aspects of Augmented Reality, and therefore serve as a better definition, AR should not be limited to head-mounted displays, nor to solely visual augmentation. Audio augmentation and, in the future, other sensorial augmentations could prove incredibly valuable. As an example, audio augmentation could be used in audio tours, real time translation and as assistance for the visually impaired, - all tools which could prove incredibly useful.

\section{Types of Augmented Reality}

The previous discussion on defining Augmented Reality (AR) touched on the two main types of displays; See-through displays and video-based displays. These displays differ in the way they allow the user to see the physical environment, which is either seen by looking through the screen, or it is recorded and presented to the viewer as a video. However, for the purposes of discussing modern $\mathrm{AR}$ research and development, these two types of AR can be narrowed further; mobile Augmented Reality, and

Head-mounted Augmented Reality display.
Augmented Reality through a head-mounted display is any AR that is presented on hardware that is over the eyes or attached to the head. This allows users to see augmented content on glasses or a screen that is directly in front of their eyes. The user can see the physical environment through the glass, and any digital content is projected onto this glass, allowing both the digital entities and physical environment to be seen through the same display. A prime example of this is the Microsoft HoloLens (Microsoft, 2016).

Mobile Augmented Reality is AR that uses a smart phone or tablet as the hardware platform. This type of video-based display uses the device's camera to live record the environment and apply computer vision to recognise the scene, either finding visual markers or recognising points in the scene to generate planes as anchors for digital content. An example of this is any smart phone running Pokémon Go (Niantic Inc., 2016). As discussed mobile AR is one of the most popular and available methods of AR. For this reason, mobile AR will be the focus of this research as the type of AR to be investigated and developed.

\section{Methods of Augmenting Reality}

There are many different methods to augment a physical environment with digital content. Most early Augmented Reality used recognisable real-world markers (Billinghurst, Kato, \& Poupyrev, 2001) (Reitmayr \& Schmalstieg, 2003). Software would train to recognise patterns and imagery as markers, then overlay these markers with digital content. Until 
recently most mobile Augmented Reality relied on this method. Early development kits, such as ARtoolkit and Vuforia, as well as AR browsers and like LayAR and Augment used this method.

More recent developments in the field have begun to push Augmented Reality in new directions. Both the above-mentioned software kits (ARToolKit, Vuforia), and recently released ones (Apple ARKit, Android ARCore) are focusing on planar tracking. This method tracks recognisable features through the camera recognising flat surfaces that could serve as anchors for digital content without using specific physical markers generated in advance (Apple Inc., 2018b). This allows developers to make content that can be placed anywhere in the physical environment, without the need to create and distribute specific physical elements. This method is not necessarily better; however, it does open the possibilities of the technology for developers and content creators.

Planar tracking could eventually give way to full spatial mapping. This tracks everything in the area through the camera and other sensors, building a full digital 3D mesh of the environment. This method is powerful and allows developers to use more information about the physical environment to create digital content. However, this currently requires very specific hardware to run, such as the HoloLens or a Google Tango device. Since they were released, HoloLens has ceased production citing further development needed and Tango has evolved into ARCore, a more accessible platform without spatial tracking, available across a variety of smart phones. This shows a current preference for accessible and cheap AR. While the future of AR could lie with specific hardware, that hardware is currently not good enough to justify the cost and investment, especially given mobile AR's recent advancements and prevalence.

No matter the method of Augmented Reality, it can be used in a number of ways, and has a number of strengths when creating content or utility. The possibilities for AR are only growing and growing for both practical tools and creative expression.

\section{Uses for Augmented Reality}

Augmented Reality (AR) has been used in many ways to present content and information while still relating to the physical world. Part of its strength lies in this adaptability and application to many different scenarios and methods of content display. It can be used to supplement information or add animated content and visuals to narratives. It can allow precise digital tools to be used within a physical environment or overlay historical visuals in a modern-day environment. It can make use of massive or small scales, without the restriction of available or functional materials. This section aims to address and explore the research in this area.

Billinghurst, Kato, \& Poupyrev's Magic Book (2001) used AR to add content and substance to a physical object. This project added an extra layer to a children's book, that was completely readable and accessible without the use of AR. However, AR was used to add a level of visual interest and moving animation. This has also been used to add more practical content and information to a physical environment. For example using AR to add contextual information to university signs, with specific 
and relevant information being added to the signs for specific users (Ajanki et al., 2011).

This use case has subsequently been developed in an artistic context by Australian artist SutuEatsFlies with the application EyeJack which commissioned artists to design sketches and animated overlays which were shown through AR (S. Campbell, 2016). This application originally supplemented a published art book but is now being used in exhibitions and other physical media.

Pacheco et al. (2015) used AR in a more spatial way, covering a large geographical area. This used AR to present digital content at the site of the Bergen-Belsen Nazi Concentration Camp. It integrated 3D reconstructions of the camp onto the site, as well as supplementary information, documents and multi-media. This use of AR allowed a large physical space to be explored by users without a tour guide. All the necessary information was contained within the iPads used to demonstrate the application. On top of this it allowed users to truly recognize the physical scale of the camp, and the buildings that were there, as they could be visualized in $A R$ at full scale, placed in their original historical location. This opens the door to large scale artistic projects that would be too expensive or impossible to develop physical sculptures for.

Bishop (2017) used AR in a number of interesting ways, showing its use in terms of contextual comparisons. Allowing video content to be displayed on posters, interviews to be embedded within images, animated content to be placed on dioramas and facial recognition to place someone in the shoes of another.

More uses can be seen can be seen in Chapter 3, where many different functions and features are explored. With many non-academic $A R$ content making use of other strengths and use cases. In mobile AR, one type of application is that of the AR browser, which allows people to upload content that can be placed in AR through a specific application. These browsers are often used for commercial advertisements and simple media content. However, it sparks an interesting question about AR as a platform for content rather than as separate experiences.

\section{Augmented Reality as a Platform}

In the context of software and development; a platform is a software environment through which smaller applications can run. Augmented Reality (AR) platforms provide the functionality of basic AR tracking and interaction for applications and experiences. This functionality can work in two ways: through AR software development kits (SDKs) or through AR browsers. SDKs only provide the functionality at a base level and require more development to publish into applications. AR browsers provide this functionality, as well as a front-facing application that allows users to upload assets that can be viewed in AR. Both of these platforms types work towards making AR more accessible to creators and consumers, decreasing the development time to create AR content.

Mobile AR browsers are very accessible for creating and uploading content, however many are generally marketed towards specific types of 
content or are restrictive in functionality. For example, LayAR (n.d.) is marketed towards creating commercial advertisements, and only uses marker-based AR, restricting content both in theme and functionality. WallaMe (2017) is another browser which is focused on allowing people to leave very simple drawings and messages digitally but at physical locations. This limits people to simple 2D content creation and rapid sketches. A study into these browsers have shown users view these applications in a negative way, citing a lack of interesting content and technical limitations preventing long term use or retention (Langlotz, Grubert, \& Grasset, 2013). As discussed in this literature review, the technology has already improved since then, and is continuing to do so. This leaves the topic of quality, engaging content, a topic which should hold interest to designers.

The success of a platform is not only measured by its immediate users, but also user retention. To achieve this the platform should be widely accessible and have a large variety in content. This content is fundamental to the platform's success and creators should not be limited by functionality or topic areas. Different content could draw different users, growing the overall user base. Consideration into the potential of an $\mathrm{AR}$ operating system or browser for any and all content is being made and some progress is being made towards this. Software companies like Apple are beginning to discuss setting standards and file types for AR content. (Deahl, 2018). Social media platforms such as Snapchat, Instagram and Facebook are also beginning to offer AR functionality within their apps, although with limited content.

$\mathrm{AR}$ as a wider platform appears to be on the way. this begs an interesting question: other than commercial advertisements and gimmicky additions to social media, what content could we see within the AR space?

\section{Augmented Reality Art}

Augmented Reality (AR) entertainment and art is one of the smaller areas of academic research into AR. Only $5 \%$ of a survey of AR usability studies from 2005 to 2014 fit into the category of entertainment, with there being no category for AR art (Dey, Billinghurst, Lindeman, \& Swan, 2018). However this field is growing, game engines like Unity 3D (Unity Technologies, 2018b) and Unreal (Epic Games, 2017) are showing support for AR content within the industry. These services also have free tiers, allowing developers to experiment with and create AR content. As previously stated mobile $\mathrm{AR}$ is already very accessible to both creators and consumers, and the addition of these tools only increases that. This makes AR a seductive medium, especially when compared to something like Virtual Reality, another piece of technology that the entertainment industry is interested in using to create interactive content.

As covered within the Uses for Augmented Reality section, AR has a range of powerful functionality and an array of strengths that could be used to create compelling artistic content. Perhaps the most obvious form of AR entertainment to touch on would be that of the AR game. Pokémon Go (Niantic Inc., 2016) is one of the most popular mobile applications on the market, it uses AR in a simple way, overlaying Pokémon creatures in whatever location they are in. While many players don't use the AR features, the game brought a lot of attention to AR and allowed a large 
number of people to experience it for the first time. One of Pokémon Go's strengths was how much context it drew from the physical location of the player, making the digital content relate to the physical environment in a very direct way. An example of this was water Pokémon only spawning near bodies of water, where they could logically live if they were physical creatures. Several games have been released in AR, but few have had the same popularity. While this is in part due to the lack of an associated franchise, it is also because many do not use the physical world to relate to the content shown. Artists have a role in broadening the horizons and opportunities of technology, pushing the boundaries of functionality, application and even permission (McGarrigle, 2014). AR art, therefore, has an important place within the landscape of $A R$ and the research into it, despite it not being a large area of research within the broader AR field.

AR has been used in works that talk to redefining public space, as well as the ownership and perception of that space (Lodi, 2014). AR art has the unique ability to be both visible and invisible, accessible and unseen. It can allow content to be seen by specific people, depending on their use of an AR lens. The location is therefore not only a physical environment but also a space for digital content and intervention. For example the We AR in MoMA piece (Veenhof \& Skwarek, 2010) directly addressed $A R$ as a medium, and how it would affect public and private spaces by placing an AR exhibition within the annex of the New York Museum of Modern Art, without the Museum's permission. Another example of AR addressing public space, is the AR Occupy Wall Street campaign (Skwarek, 2011). After protests were banned from protesting on wall street itself, $A R$ protesters placed work within the financial district through $A R$.
This sparks an interesting discussion around AR's effect on policing and governing space. Does the digital space also need to be policed as well as the physical space? (Skwarek, 2014).

$\mathrm{AR}$ artworks relate the viewer, the environment and digital media all within the same frame. The viewer might need to physically interact with the media, moving and stretching to change their view of, and interaction with, the media content (Lichty, 2014). Artists could also explore users' face and body as a canvas, with content being placed within the context of a person's physical body. (Lichty, 2014). This creates a sense of true relation with the content, literally changing that person's perception of self or putting them in the shoes of another. AR artworks could also make direct use of large scale data within artistic or political content, due to the fact that the work could be connected online and be constantly updating (McGarrigle, 2014).

AR art actively dialogues with the space it is in, drawing context or subverting the expectations of the space. (Garbe, 2014). Limiting the number of locations, and therefore contexts AR art could draw on would limit an otherwise expansive potential. AR art should therefore not be limited to a single type of location, such as galleries or institutional spaces. For these locations, how would one curate digital content that anyone could upload anywhere and view? Does this completely breakdown the barriers of curation? Or merely shift them into a digital format more within the creators control (Garbe, 2014)? In either case this offers a more democratised approach to the viewing of art, removing the gatekeeping and barriers to entry that come with a gallery space. There are, 
unfortunately, still the barriers around access to technology, and literacy with that technology. This is limited by the prevalence of mobile AR, which gives access to AR to anyone with a smart phone. For creators there is the additional barrier of development ability which is notable, however with free development tools such as Unity 3D (Unity Technologies, 2018b) and Unreal Engine (Epic Games, 2017) that both support AR development, this barrier is lessened.

AR then, draws strength from locations and viewers, has a low barrier to entry, and has little to no curational gatekeeping. AR has been used to engage viewers with issues, protests and public space. It is in these core foundations of AR art that a parallel begins to emerge with an older and more storied art form: Street Art.

\section{Understanding Street Art and Graffiti}

In the late 1960s, on the streets of New York City, the subculture of graffiti emerged. Graffiti is a cultural practice aimed at creating a shared and democratic visual space for expression (Dickinson, 2008). However, at the time the city's government was strongly aligned with the business community, resulting in policy that was aimed at radical privatisation of the public sector, and claiming centre city as a place for the whitecollar New Yorker (Dickinson, 2008). This not only led to the public opposition and demonization of graffiti, but also its definition as a criminal act. This forever aligned all graffiti writers as vandals, thugs and criminals (Dickinson, 2008). This did nothing to stop the practice, and instead encouraged writers to use graffiti as a way to reclaim profit driven privatised spaces. Graffiti writers used their medium to critique policies that encourage profit-driven commerce within a public space. Policies that could, for example, legitimise corporate advertising at the expense of art (Sarah Banet-Weiser, 2012). This culture and practice soon spread from New York, and could be found throughout the world, used as a medium for public responses to political issues (Ferrell, 1995).

From this early graffiti culture and practice, the practice of Street Art developed. Rooted in similar values and ethics, Street Art also works to reclaim the public space and identify the role of creativity within the that space (Sarah Banet-Weiser, 2012). The defining characteristic of Street Art as opposed to graffiti, is that its location and use of the street as an artistic resource is internal to its meaning (Riggle, 2010). Conversely, Graffiti uses a public surface in a more general way, usually for the publicity the street provides. Street Art relies on its location, and loses all meaning when it is moved from its original location (Riggle, 2010). In using the street so inherently, it relinquishes all control to the street and the public that uses it. Street Art is therefore designed for the street and can use a variety of materials to place it there (Ross, 2016).

\section{Perceptions on Street Art}

Street Art exists on its own, outside of the artworld, and away from gallery spaces. These spaces act as gatekeepers and curators for high art, whereas Street Art's significance and meaning relies on being outside of that world (Riggle, 2010). Private collections and galleries desiring Street 
Art to collect and display, do so in a futile effort. Removing the art from its location results in a loss or shift in meaning, resulting in a loss of its essence. This lack of essence and shift in meaning could be compared to Walter Benjamin's (1935) theory about art and reproduction, which argues that losing essence and meaning results in a loss of authenticity. Without this authenticity is it the same work that the collectors desired so much? Many attempts at displaying Street Art within a gallery context have resulted in conflicts between the ideology and style of Street Art , and that of the gallery space (Bengtsen, 2015). As Street Art is being accepted into these spaces, a trend in commissioned and legal Street Art is also growing.

Banksy is a Street Artist who's politically charged work has sought to shed light on issues ranging from homophobia, war and violence, using the public street as a canvas to make provocative statements and elicit responses (Banet-Weiser, 2012). The trajectory of his career is one that highlights the positive shift in public perspective towards Street Art. He has become a globally recognised artist with his work found internationally and becoming increasingly valued and sought after by both public and private collectors. His name has become a brand, supported by brand culture and the creative economy despite his strong critiques of these cultures. Through his brand his work continues to push the idea of the "free enterprising individual", the artist who does not ask for permission but takes and repurposes (Banet-Weiser, 2012, p. 95).

There is still tension between Street Art and legitimacy, acceptance and legality. Galleries and institutional spaces are placing prices on the work, presenting it in their own spaces away from the street. Advertisers and brands are using Street Art in campaigns, brands and clothing (BanetWeiser, 2012). This legitimisation and commercialisation of Street Art culture raises debate in its community. In this form, no longer using the street in a materialistic way, this art is no longer Street Art (Riggle, 2010). However, what is clear, is that the public is showing an appreciation for Street Art, and the public perspective has shifted away from the label of vandals and criminals in recent years (Rowe \& Hutton, 2012). A New Zealand study into young peoples' perspective on graffiti and Street Art showed that even those who viewed it as a criminal act, still labelled it as an "urban art-form that should be valued in some circumstances" (Rowe \& Hutton, 2012)

So far this literature review has covered the concepts of Augmented Reality and art using that medium, as well as Street Art and the perceptions towards it. The remainder of this literature review seeks to draw attention to the parallels in these concepts and align the two fields in a way that situates the trajectory of this research.

\section{Augmented Reality Street Art}

Augmented Reality (AR) is strengthened by a physical location from which it can draw either a material or social context. This is not dissimilar to how Street Art uses the street as an internal part of its meaning (Riggle, 2010). A gallery space would limit the possibilities of AR content, and 
change the meaning of Street Art, resulting in a weakened experience of the work. Compared to other digital interactive mediums, AR is a very accessible medium to both creators and consumers. It can be developed for a smart phone, a device many already own. Similarly, Street Art has very low barriers to entry, with the material cost being almost non-existent to a person with an idea for creating work with the street.

By marrying the ideals and parallels of Street Art and AR content, AR Street Art has the ability to use digital content to contribute towards the visual language of the public space (Gwilt, 2014). AR Street Art has the potential to build on the visual language of Street Art and the urban environment, as part of an ongoing fusion between analogue and digital content (Gwilt, 2014). AR street art could then be seen as Digital Street Art, using the possibilities and features of AR technology, while still using the street as its location from which to draw context. Mobile AR allows this use of AR to be very accessible to both creators and consumers, due to its use of a smartphone, which is already a device used and prevalent in the public space.

Remaining outside the gatekeeping and barriers to the artworld, Digital Street Art could draw inspiration from both traditional Street Art practice and AR Art. It could work to reclaim the public space making social commentary, political critiques and adding a spark of interest and life. The prospect of combining these two practices holds implications for the artists, and the tools at their disposal. Using AR, a Street Artist could spend more time and energy on a non-commissioned piece without fear of persecution. Using the street allows the AR artist to make very public works that serve to dialogue with the public and the space its within. It is important to note that this practice would not replace traditional Street Art, but rather supplement and support it. A new way for creating Street Art that still engages with the public space to make provoking statements and elicit responses.

\section{A Platform for Digital Street Art}

The combined practice of Digital Street Art does lead to a further question: How would this work be exhibited to viewers? From a technological perspective, all that is needed to view Augmented Reality (AR) content would be a mobile phone. However, there is still the matter of distributing the content itself to these devices. Currently AR content and experiences are distributed through the use of individually developed applications or through AR browser applications. Individually developed applications for every work of Digital Street Art is inefficient, viewers would have to download a new application for every artwork, and artists would need to have development skills and resources to create these applications. Current AR browsers are targeted towards specific commercial markets or are limited in functionality. A space for a new AR browser exists, one that allows the Digital Street Artist to fully explore their creativity, unlimited by content specifications and functionality. A platform for Digital Street Art that follows the culture of Street Art that uses the technology of mobile Augmented Reality to place it in the public space. 


$$
\text { Chapter 3: }
$$

Free Experimentation 
This thesis combines the research fields of Street Art and Augmented Reality (AR) to propose AR as a method for creating Digital Street Art. The previous chapter worked to understand the parallels and contrasts between these fields and situate the context of this research. The remainder of this thesis draws on this academic research and applies it to a more practical design methodology. Research through design as an action - reflection approach is used to consider the creation of Digital Street Art using AR, as well as implications for a potential platform through which Digital Street Art could be hosted.

As discussed in the previous chapter, Digital Street Art and any platform for it should exist in the most accessible way possible. To limit gatekeeping and barriers to entry, mobile AR was used throughout the development of this thesis. Currently this is the most available type of AR however future development and adoption of head-mounted AR displays could change this.

This chapter documents the initial steps taken to understand how Digital Street Art could be created using mobile AR. These free experiments investigate the technology, precedents and context of creating Digital Street Art using AR in Wellington, New Zealand. The final findings of this chapter inform the further developed design outputs documented in Chapter 4. 


\section{Precedent AR work}

To begin practically exploring Augmented Reality as a medium, some precedents were explored. These precedent works offered insights into possible themes and topics, as well as technical approaches to content delivery.

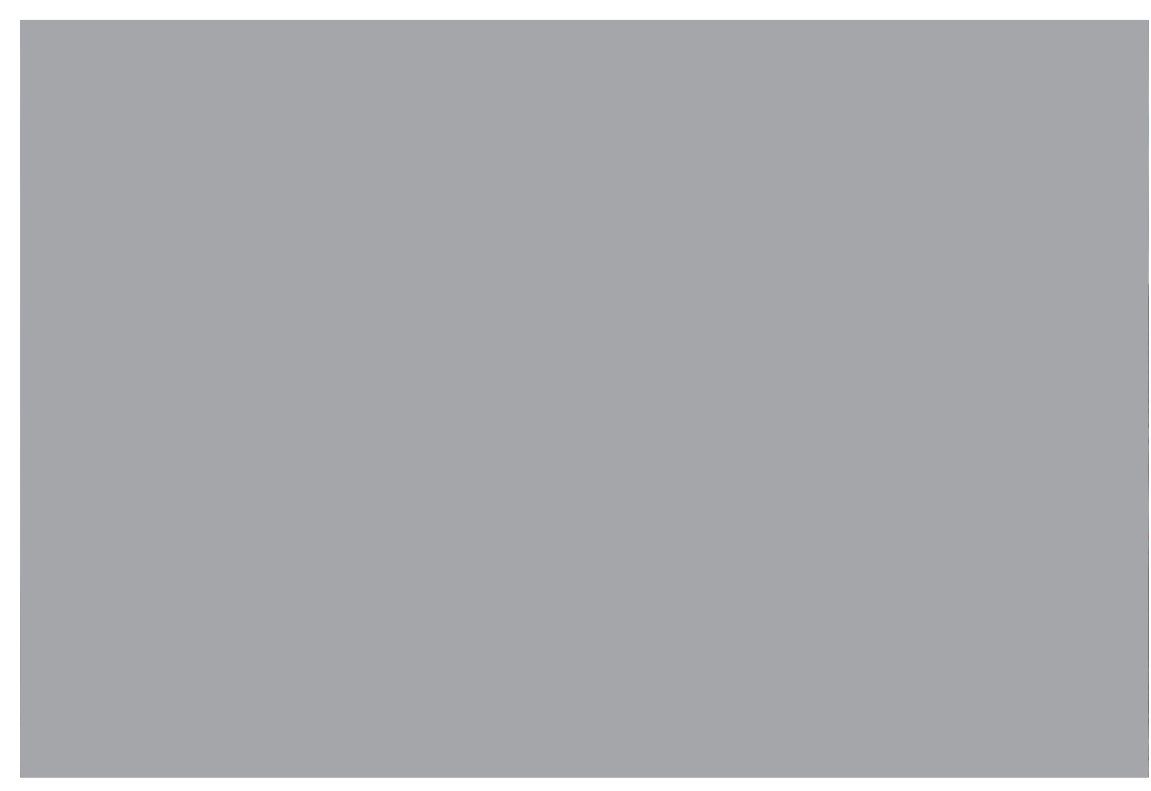

Figure 2: ARtvertiser being used to replace a large-scale billboard

\section{ARtvertiser}

Julian Oliver, Damian Stewart, Arturo Castro

2008

(Oliver, 2010)

Describing itself as an "Improved Reality", ARtvertiser is a software platform that replaced billboard advertisements with art in real-time. Using the original billboard as a recognisable marker, ARtvertiser could then replace the existing advertisement with alternative media, like images and video. This project aimed to consider places like Times Square or Shibuya Tokyo, each with dense advertising, as potential exhibition spaces. The artists considered billboards and advertisements as "read-only" to the public, with only corporate advertisers having "write-access". By using Augmented Reality (AR), the developers shifted the control to the public, allowing the viewer to choose what they see by looking through the lens of AR. This work addressed the ownership of public visual space and sought to reclaim it through a digital lens.

\section{Insights:}

Marker-based tracking has been replaced by planar tracking in many places, however it still has value in certain circumstances, and should not be ignored. While Mobile AR may be inherently linked to the use of a technological device, corporations, even those that manufacture such devices should not be beyond reproach and commentary. 


\section{AR Occupy Wall Street}

\section{Mark Skwarek}

2011

(Skwarek, 2011)

In conjunction with the occupy wall street movement in September 2011, AR Occupy Wall Street was an Augmented Reality (AR) activism project that supplemented the physical protests. It is possibly one of the most widely known and discussed AR activism projects (Skwarek, 2014), in part due to the fact that it tied so heavily to a very public physical protest. The protest was occupation of the New York Stock Exchange with AR content. The area was made off limits to physical protest and so AR was used to project the global community's voice into the location (Skwarek, 2014). Over 25 artists contributed 400 digital works to the location, allowing messages and works of art from all over the world to be added to the protest. Not only did AR allow for protests within a blocked space, but also allowed contributions from people that could not join the physical protests.

\section{Insights:}

Street Art has a long history of protest works, and AR Street Art should be no different. Protest works offer a number of themes for artists and designers to address. Contributing to locations from across the world is a unique feature that $\mathrm{AR}$ can allow, and this should be considered for both content and platform.

\section{We AR in MOMA}

Sander Veenhof and Mark Skwarek

2010

(Veenhof \& Skwarek, 2010)

This work was aimed at exhibiting Augmented Reality Art in its "proper context": a contemporary art museum. This experimental exhibition was part of the Conflux Festival, an annual New York festival dedicated to the psychogeography practice. This practice studies the laws and effects of the geographical environment on the emotions and behaviours of individuals. By placing an uninvited AR exhibition into the annexe of the New York Museum of Modern Art, the organisers of the event aimed to address a contemporary issue, caused by the rapid rise of Augmented Reality usage. 'What is the impact of AR on our public and private spaces?' (Veenhof \& Skwarek, 2010).

Insights:

This work initiates an interesting discussion around AR in the private space. Should AR be blocked from these spaces? Or is blocking locations blocking the freedom of the artist? 


\section{Jeff Koons's 'Balloon Dog' AR}

Jeff Koons, Snapchat

2017

(Chow, 2017)

In 2017 snapchat partnered with Jeff Koons to bring his sculptures to life in Augmented Reality (AR), placing them around international landmarks using geolocation. The aim of this collaboration was to inspire artists, by showing that giant sculptures could be placed anywhere in the world using digital methods. People could also take photos of and with the sculptures, sharing them in the Snapchat app. These sculptures were tied to landmarks in a number of cities, such as the Eiffel tower in Paris, or Central Park in New York. These locations were well known and also allowed people to compare the scale of the digital works to known landmarks. However, artists responded to this project with criticism, questioning the way access to the digital artwork was restricted to a big company, and saying that 'we need to start discussing how much of our "virtual public space” we want to give to corporations' (Codrea-Rado, 2017). Again, the ongoing debate around public space is present. As a response, artist Sebastian Errazuriz created a version of the work covered in graffiti, placing it at the same location within his own AR application (Codrea-Rado, 2017).

Insights:

Should AR Street Art encourage the ephemerality found in physical Street Art? This would allow artists to cover other creations, however because these works are digital, there could be ways of preserving the content underneath. The involvement of commercial work and corporations are not well received by many artists and would have to be considered carefully. 


\section{Standing Ovation: Half a Century of Downstage}

Theatre

Jonathon Bishop, Seth Corker, Stefan Peacock, James Penman and Gerrit van Rooyen

2016

(Bishop, 2017)

This exhibition focused on using Augmented Reality to tell the story of six plays presented by the Downstage Theatre company in New Zealand. The aim was to use content from within the collections of the National Library of New Zealand to create artistic reinterpretations of the plays. These reinterpretations used both physical and digital content from the collections, using AR to align the two. While this work was in a gallery and did not draw from the context of its location, it did use the physical content found within the collections of the library as the base for the context. Using marker-based AR the works added video and audio content to physical photos and images, as well as adding animated stories to physical dioramas. Using facial recognition one of the designs transposed the face of one play's subjects to the face of the viewer.

\section{Insights:}

Facial recognition is a feature that could be used within AR Street Art, as is audio augmentation and video content. AR Street Art could draw from histories and stories from the location they are in, instead of solely focusing on current themes and content.

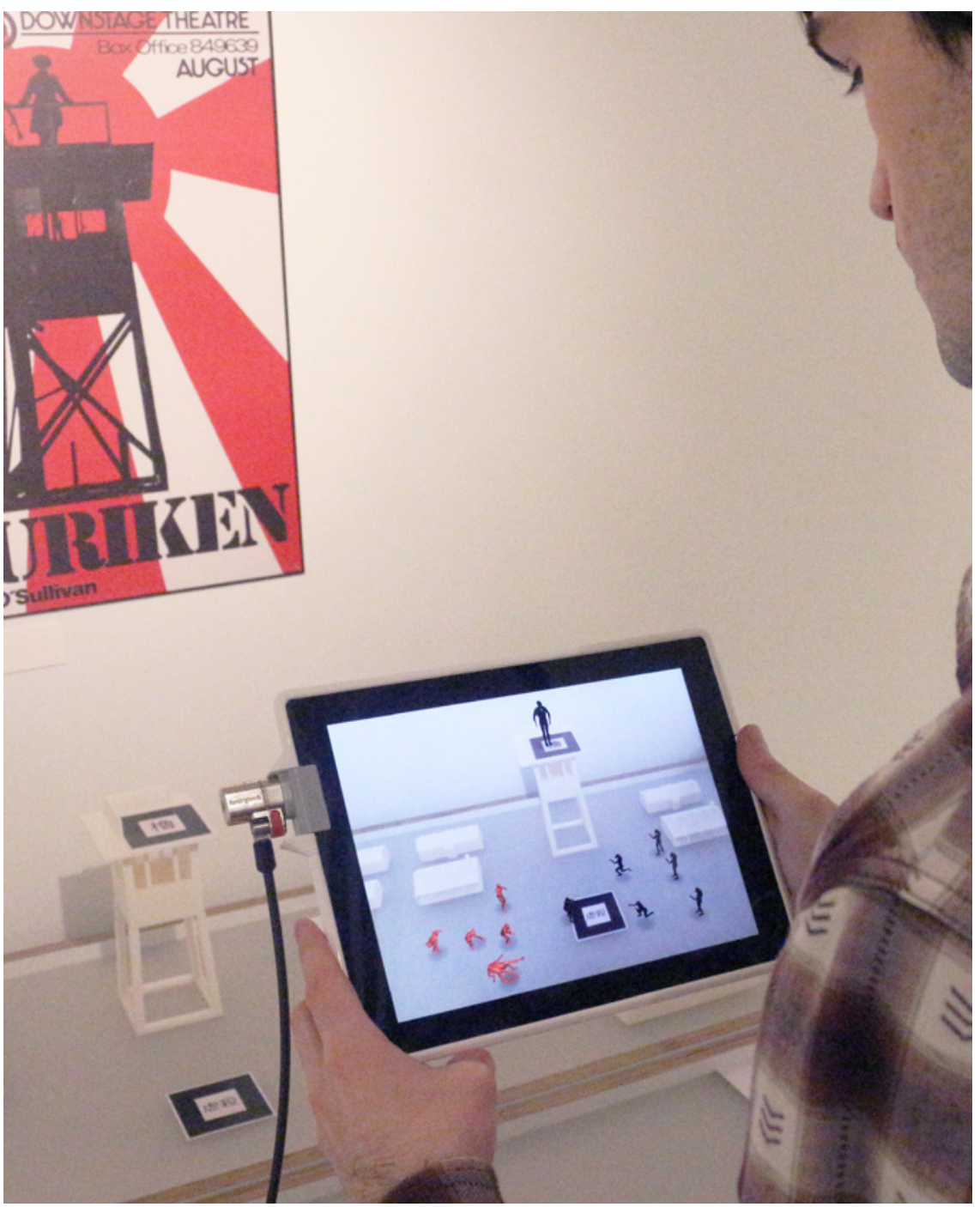

Figure 4: Untitled Photo showing one of the Downstage Exhibition works 


\section{Investigation of available tools}

For the development of Augmented Reality experiences and software, a number of tools and software development kits provide the base functionality and connecting through APIs to a number of general development tools like Unity3D, which could be used to speed up development.

\begin{tabular}{|l|l|l|l|}
\hline \multirow{2}{*}{ AR SDK } & Features & Postives & Negatives \\
(Apple Inc., 2018a) & Marker based tracking, & $\begin{array}{l}\text { Well supported and Documented, } \\
\text { Frequently updated, including plugins to } \\
\text { other software, } \\
\text { Facial tracking (iPhone X only) }\end{array}$ & $\begin{array}{l}\text { Limited to Apple devices with A9 processor running } \\
\text { iOS } 11 \text { or later. } \\
\text { (iPhones later than 6s) } \\
\text { (iPads later than 2017 and iPad Pros) }\end{array}$ \\
\hline $\begin{array}{l}\text { Google ARCore } \\
\text { (Google LLC, 2018) }\end{array}$ & $\begin{array}{l}\text { Planar tracking, } \\
\text { Marker tracking, } \\
\text { Multiplayer. }\end{array}$ & $\begin{array}{l}\text { Constant updates and available device } \\
\text { list is expanding. }\end{array}$ & $\begin{array}{l}\text { Low number of compatible devices. } \\
\text { sparse documentation. }\end{array}$ \\
\hline $\begin{array}{l}\text { Vuforia } \\
\text { (PTC Inc., 2018) }\end{array}$ & $\begin{array}{l}\text { Marker tracking, } \\
\text { Planar tracking. }\end{array}$ & $\begin{array}{l}\text { Any android or apple device. } \\
\text { Integrated into the unity engine. }\end{array}$ & $\begin{array}{l}\text { Integrated into Unity3D, planar tracking is new. } \\
\text { Free for development and testing but paid to } \\
\text { distribute or publish. Watermark present while using } \\
\text { it. }\end{array}$ \\
\hline
\end{tabular}

Based on device availability, cost and developer support, Apple ARKit was chosen as the SDK to develop AR content with. This does limit any developed content to iOS devices, however this can be expanded to include more devices at a later stage. 


\section{Initial Constraints and Technological Considerations}

\section{Hardware:}

Mobile Augmented Reality was chosen as the method for Augmented Reality, due to its availability and prevalence.

Due to availability, an Apple iPhone 7+ was used for development and was kept updated until iOS 11.4 using ARKit 1.5. As such, this is the targeted device for all software development, future development could result in a cross-platform prototype.

\section{Software:}

\section{Unity 3D}

Unity 3D is a game engine that can develop for iOS and Android. As well as being a piece of software I am familiar with, using a game engine saved time, allowing me to focus on the design and development of the specific designs rather than having to build base functionality from scratch.

(Version 2017.3.1f1)

\section{XCode}

$\mathrm{XCode}$ is the proprietary development tool for building applications for iOS, regardless of development tools this must be used to validate the application and install it onto an Apple device.

(Version 9.4) 


\section{Functional Experiments}

The aim of the initial experimentation was to test the potential of ARKit and its functionality. Understanding the technology resulted in a better understanding of the limitations and scope of development for the final designs.

The tracking in ARKit creates correspondence between the physical and digital spaces using visual-inertial odometry (Apple Inc., 2018b). This technique combines motion sensing data with computer vision analysis of the scene visible to the camera. Notable features in video frames are recognised and then tracked across frames, comparing this to the motion data creates a model of the devices position and motion, as well as the contents of the scene in a rudimentary way. (Apple Inc., 2018b)

In ARKit the axes follow right hand convention: $\mathrm{X}$-axis points to the viewers right, the $Z$-axis points towards the viewer and Y-axis points up. Understanding this allows the digital content and physical environment to be aligned, meaning that content can be digitally generated in the correct orientation.

The tracking and plane generation can be seen in Figures 5,6 and 7. Points are generated from locations of high contrast and detail, generating a sparse point cloud of spatial data in the scene, recognisable in these figures as yellow dots.
Flat areas are then recognised based on these trackable points and digital planes are generated in the software. These planes can then be used to place digital content and align it with the physical space.

In testing rough surfaces generated more points of interest, allowing planes to be recognised faster. Low physical objects were sometimes overlapped by the plane, as shown by Figure 7. Horizontal planes were recognised quicker in the application, with more stable tracking. Vertical planes were slower and unreliable, occasionally resulting in objects hovering off the surface of physical plane. This was made more obvious by the ease in which the viewer can view this plane multiple angles. This created a visual disconnect between the digital content and the physical environment. 


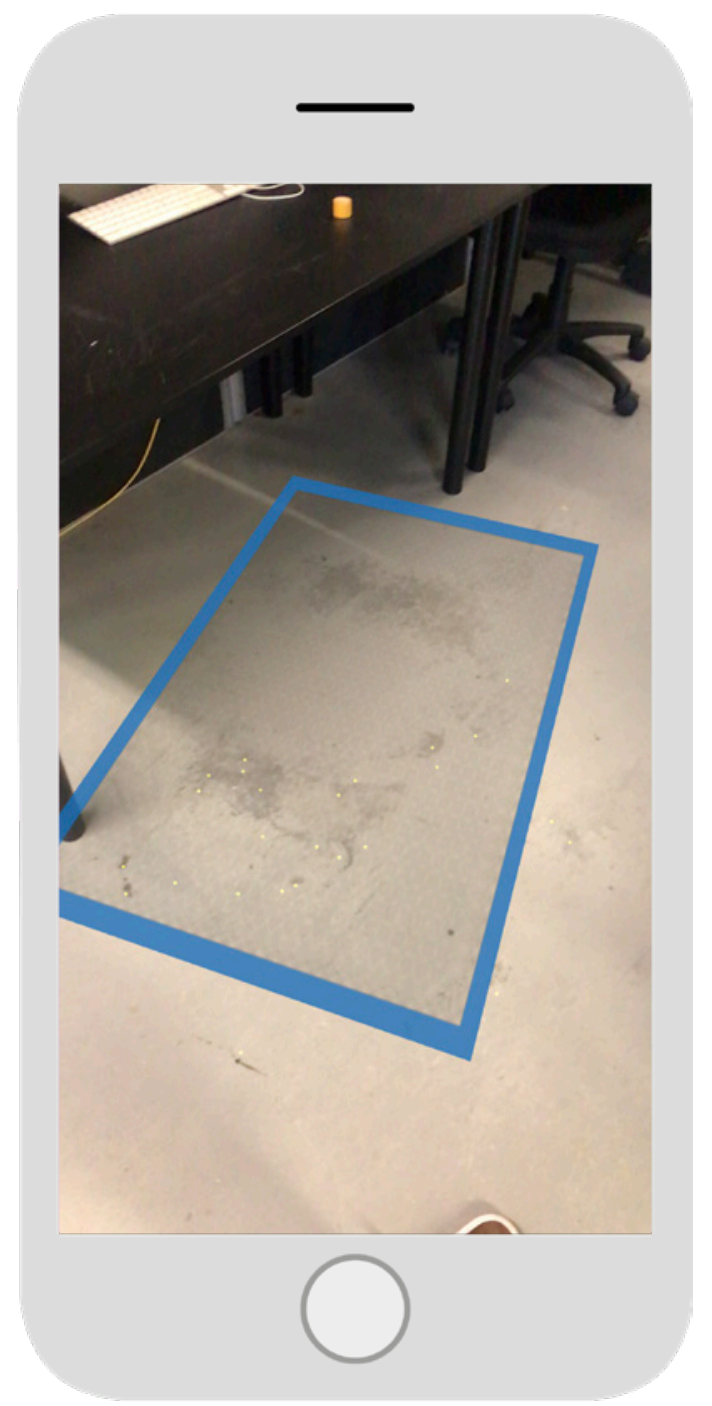

Figure 5: Uniform horizontal plane generated in ARKit

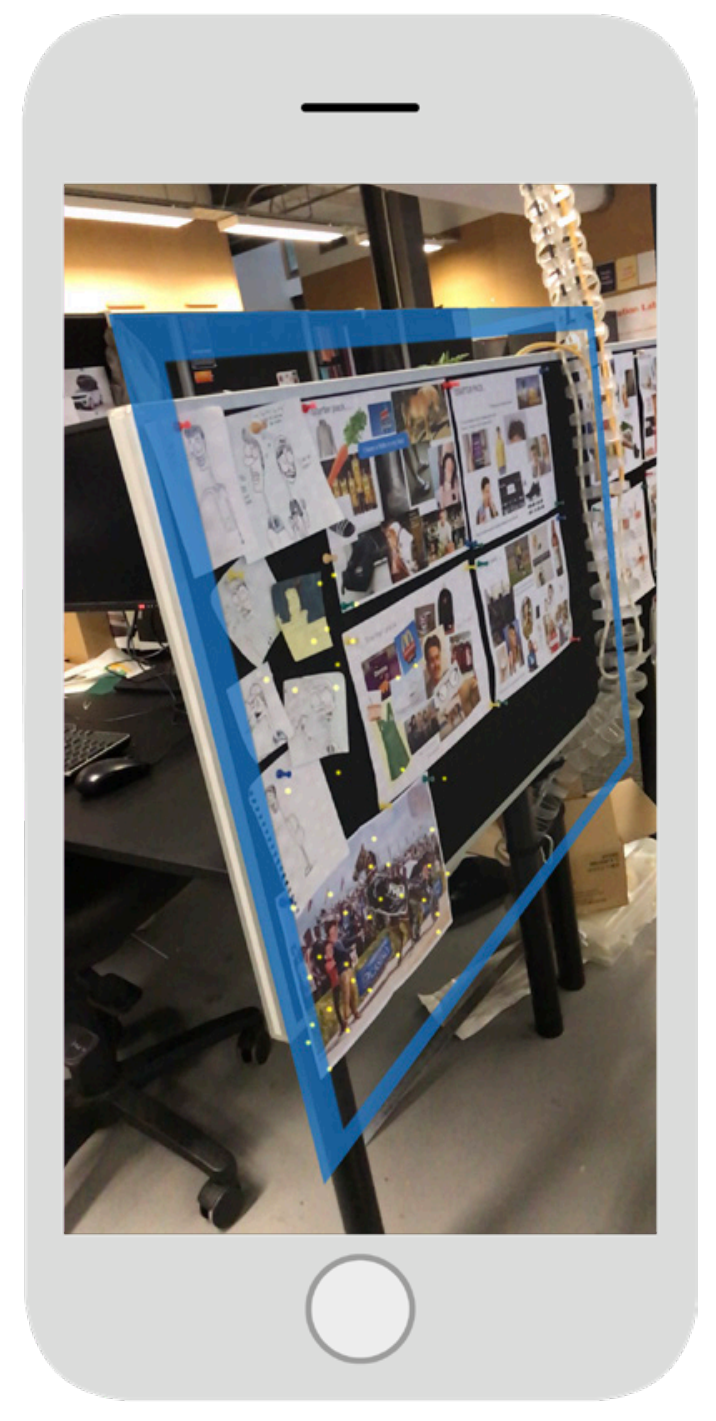

Figure 6: Uniform vertical plane generated in ARKit

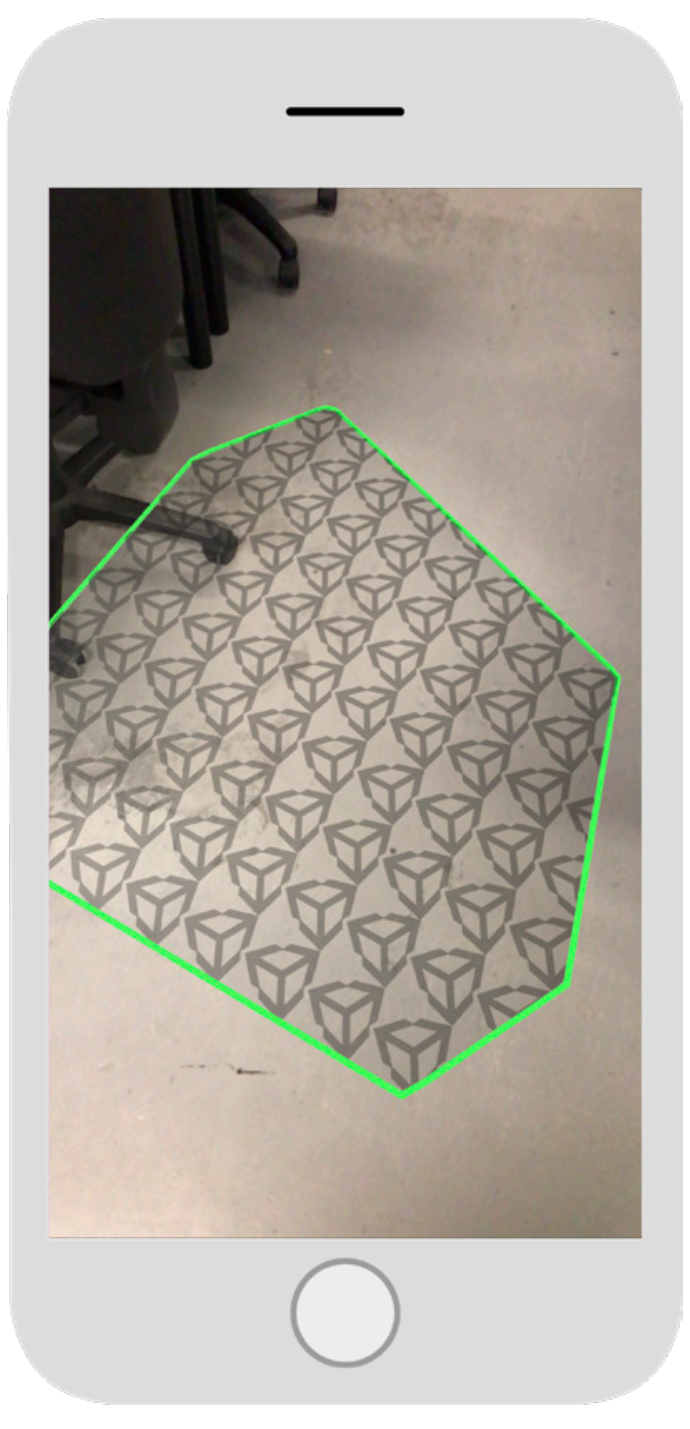

Figure 7: Non-uniform horizontal plane generated in ARKit 


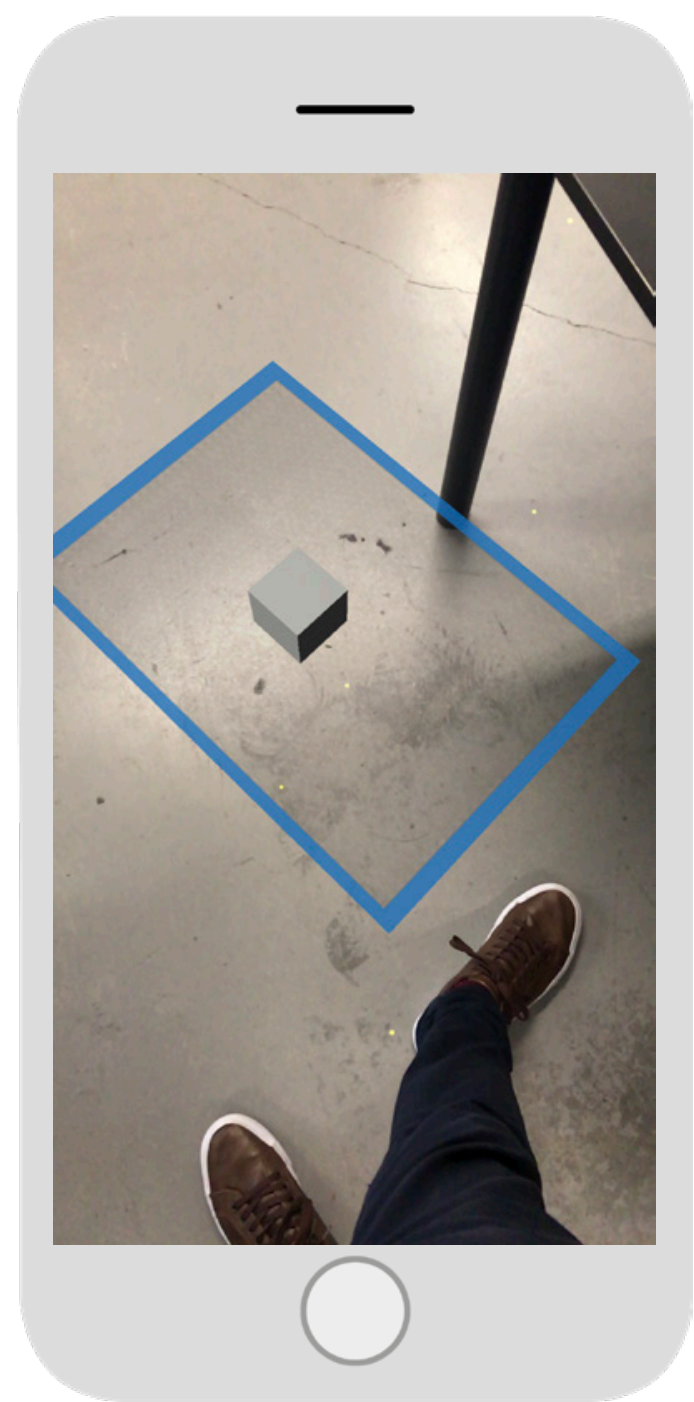

Figure 8: Primitive cube placed in $A R$, anchored to a horizontal plane

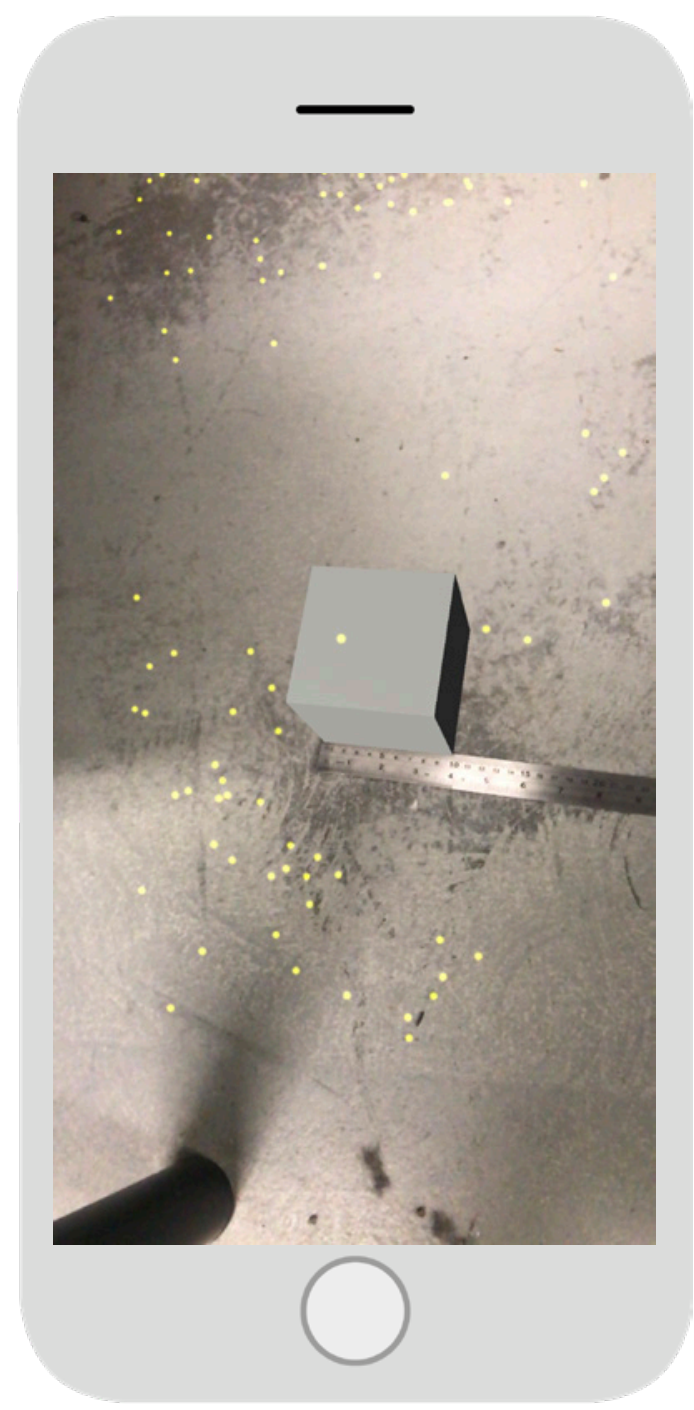

Figure 9: $10 \mathrm{~cm}^{3} \mathrm{Cube}$, digital and physical scale aligned

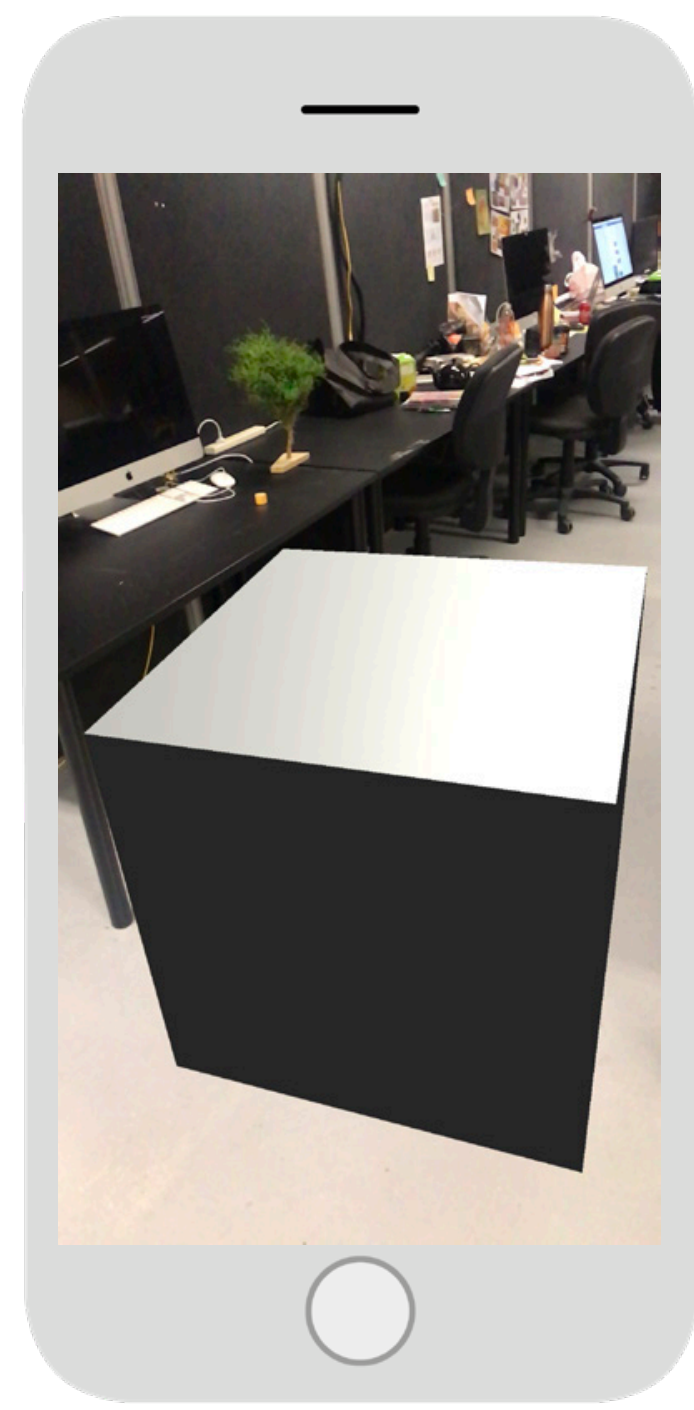

Figure 10: $1 \mathrm{~m}^{3}$ Cube showing larger scale object in $A R$ 
The generated planes allowed for digital assets to be placed, aligning them with the physical environment. Scale tests were carried out to determine what different sizes would look and feel good in AR. Unity works in meter measurements that translate 1:1 to the physical environment, this can be seen in Figure 9. From here tests were carried out to determine how different scales of digital content would feel in AR. $10 \mathrm{~cm}, 20 \mathrm{~cm}$, $50 \mathrm{~cm}, 1 \mathrm{~m}$, and $10 \mathrm{~m}$ cubes were tested in AR. This showed that small assets could be placed into the scene easier, with less regard to the physical environment. Larger objects began to overlap the tracked planes at their boundaries, resulting in models that visually clipped through physical objects. This is particularly apparent in the $\mathrm{X}$ and $\mathrm{Z}$ axes as there are more objects in this physical space. However, when testing in completely open space, most scales were stable in terms of tracking and relationship to the physical environment.
From these tests it became clear that while any size and shape could theoretically work within the AR scene, limitations would instead come from the physical location. Next different forms were investigated, stretching the bounds of specific axes. Figures 11, 12 and 13 show these tests. These shapes in particular were used due to their relation to potential shapes for specific content, adding a digital wall, tall poll or new floor to the scene.

Each of these tracked accurately enough to be stable, although the large flat object did suffer the most, hovering above the ground slightly, and shifting when adjusting the camera. Large flat objects would need to be considered with great care, even within open physical spaces. 


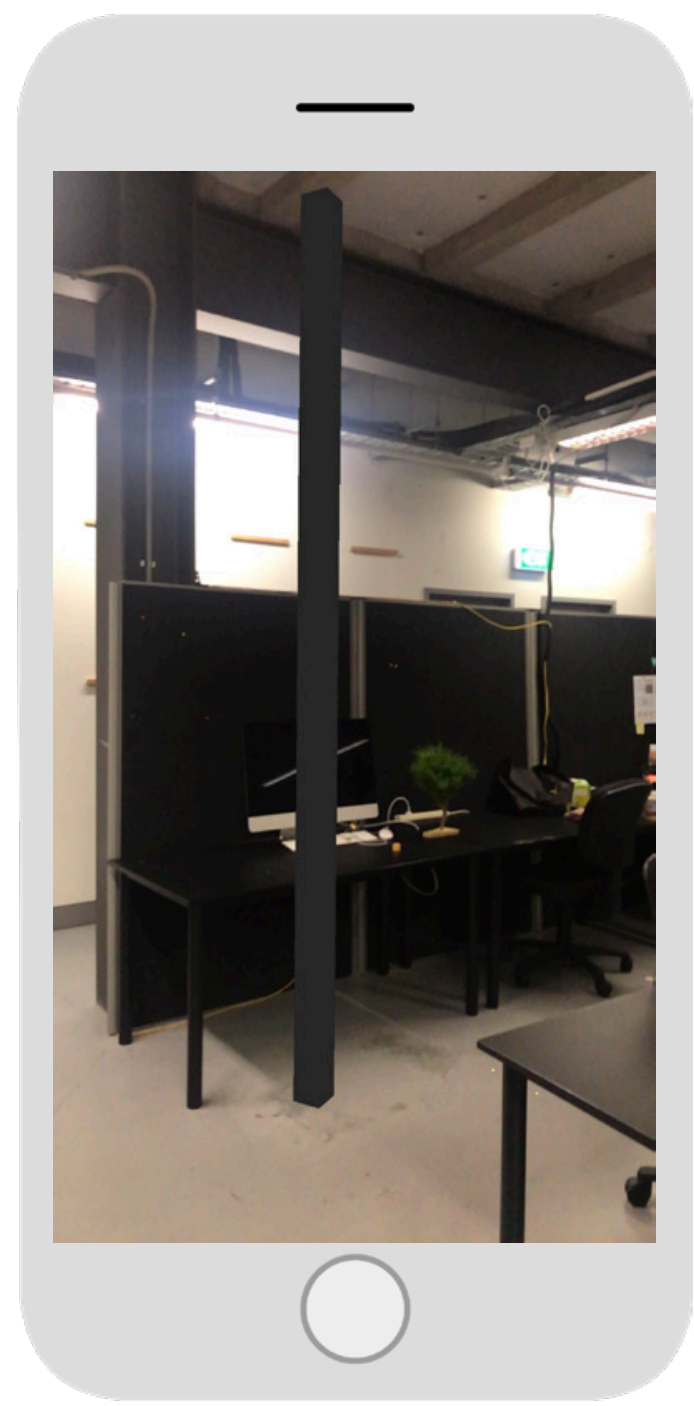

Figure 11: Tall poll placed in AR, testing height allowances

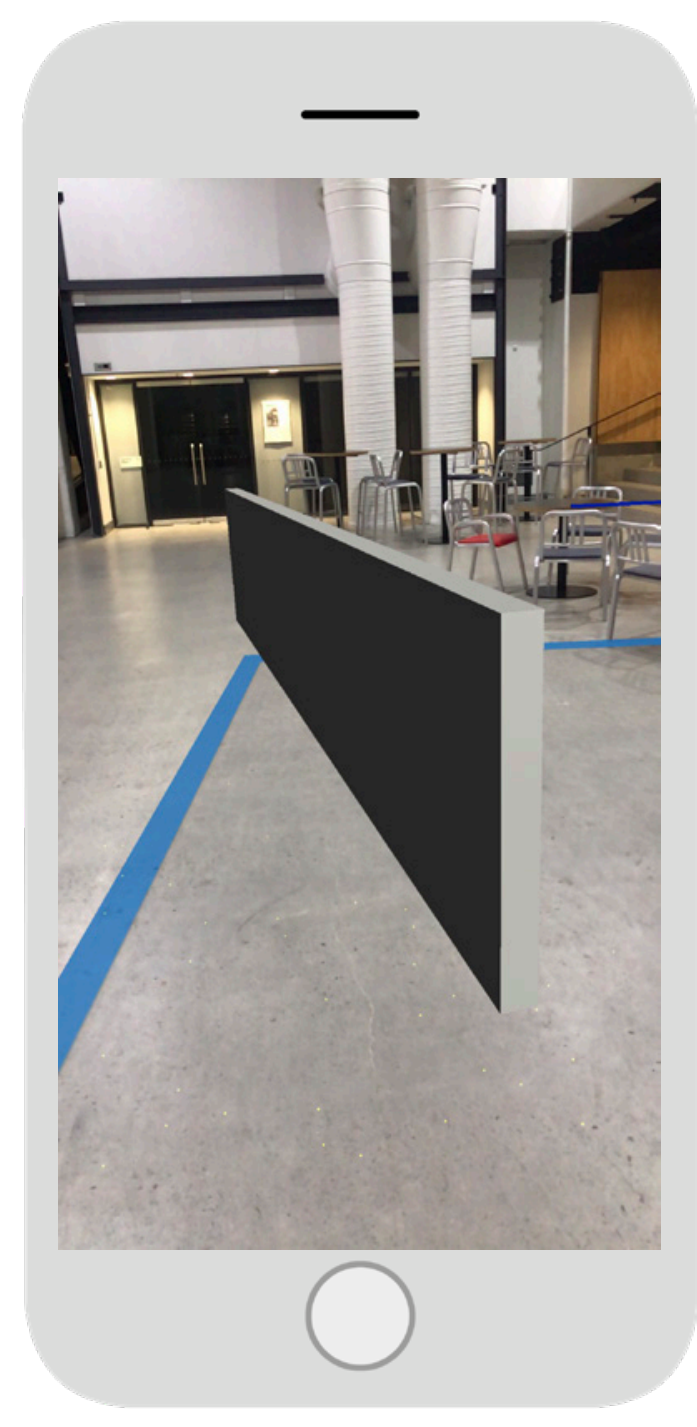

Figure 12: Large wall like object placed in an open space

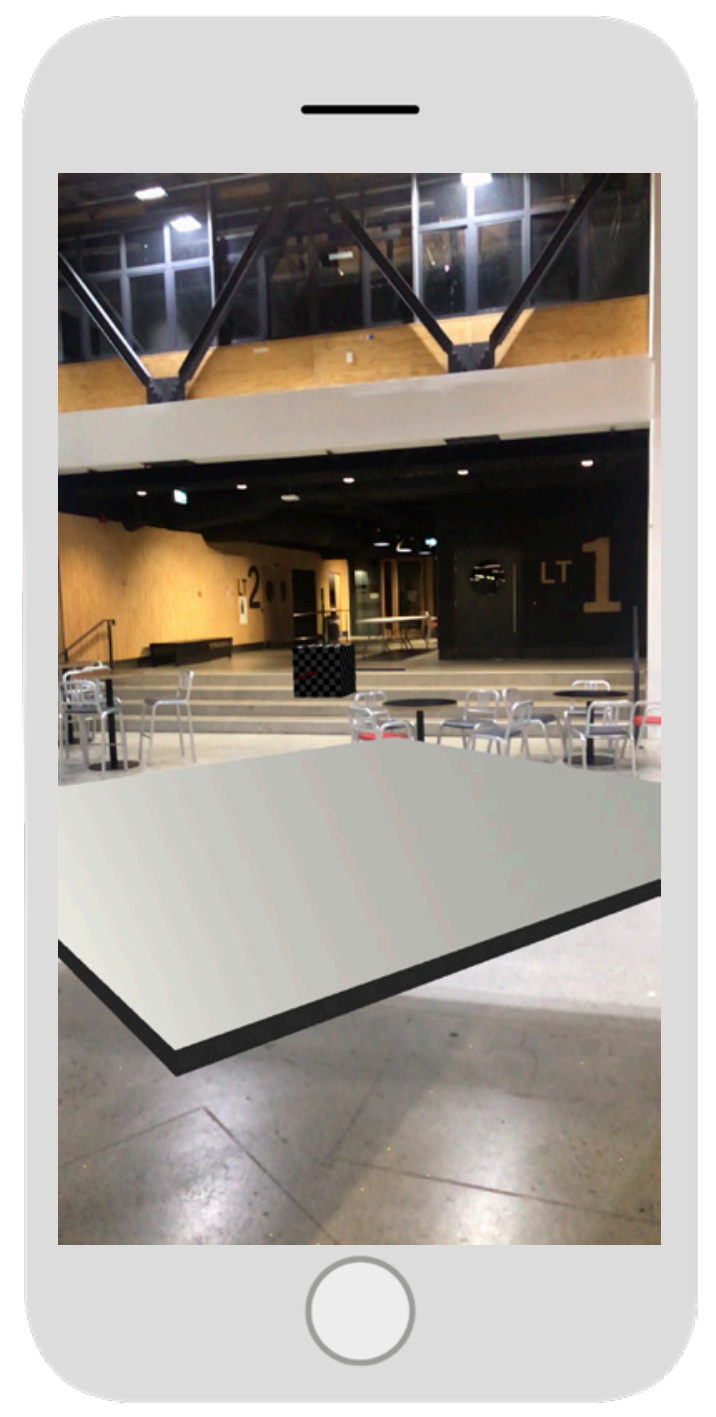

Figure 13: Extra large object no longer tracking accurately 


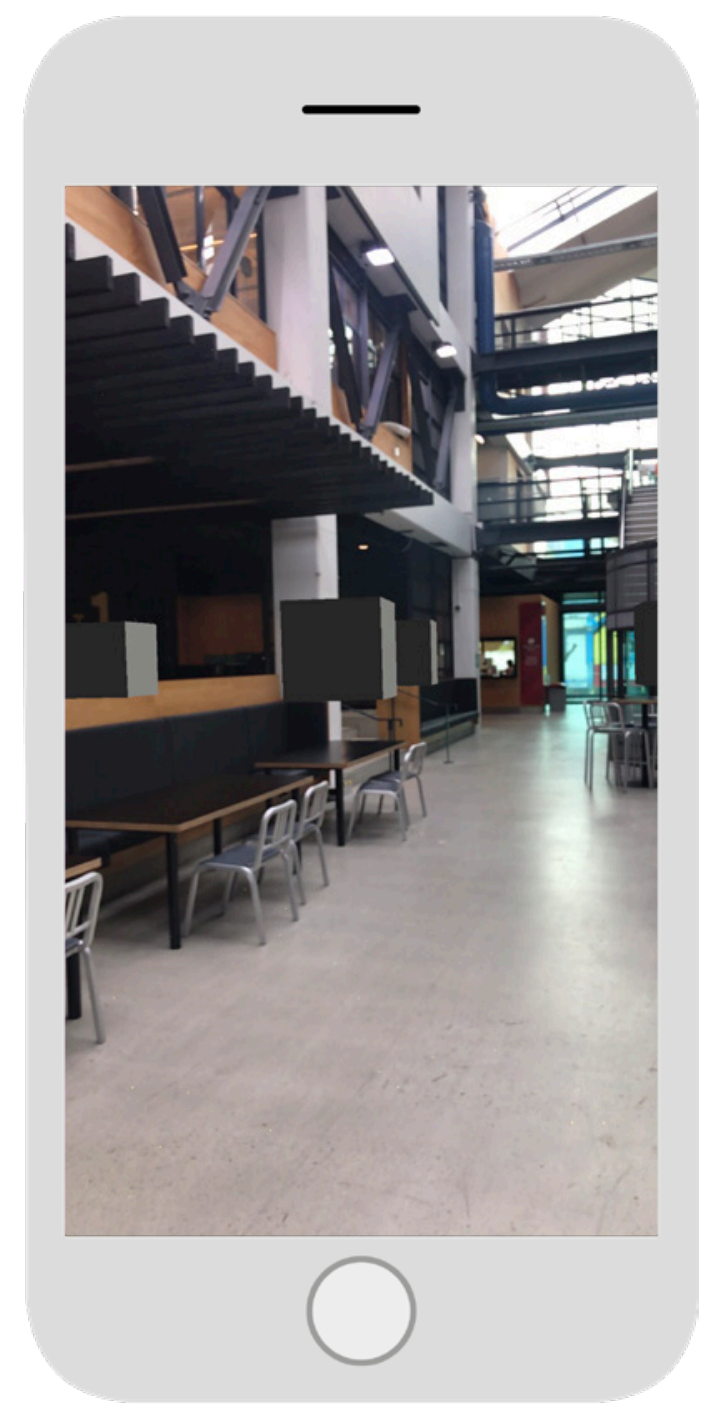

Figure 14: Cubes anchored to users original position, remaining in place once the user moves

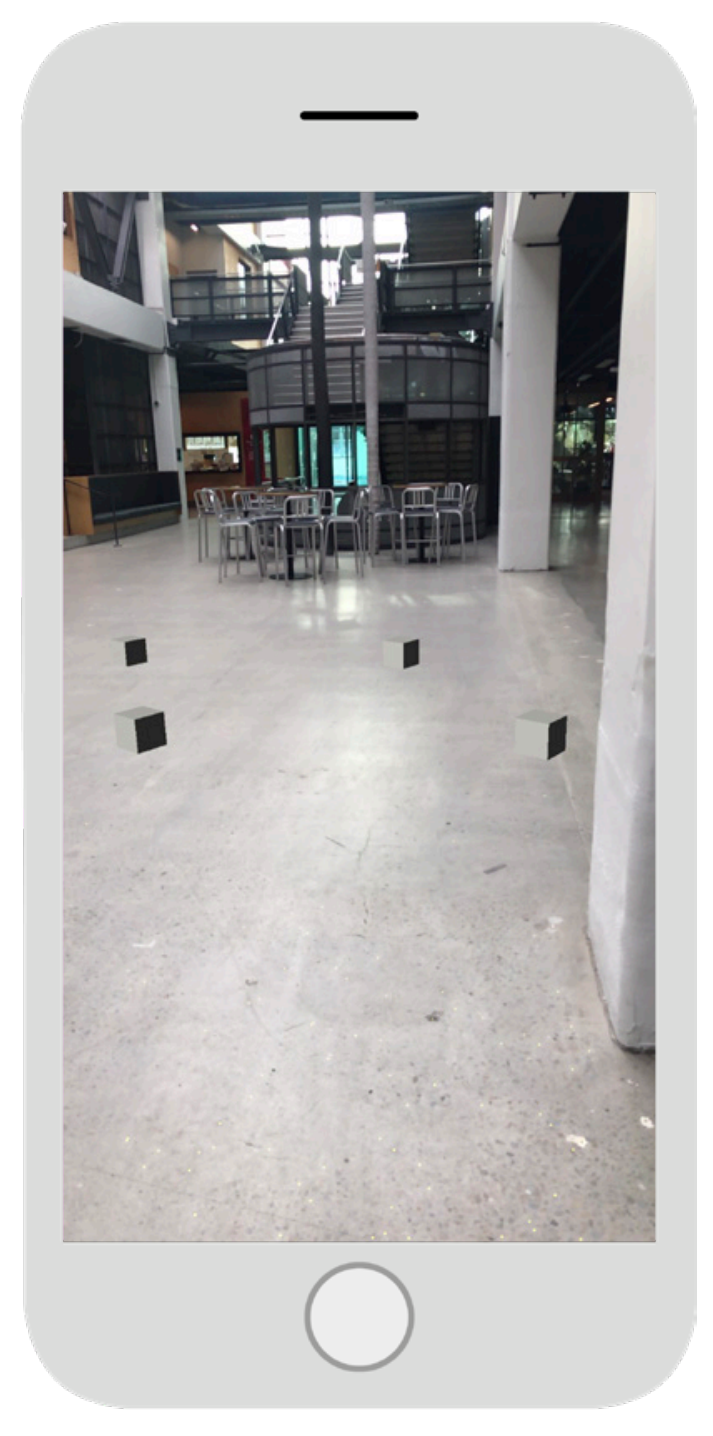

Figure 15: Instantiating many objects spread around the tracked area

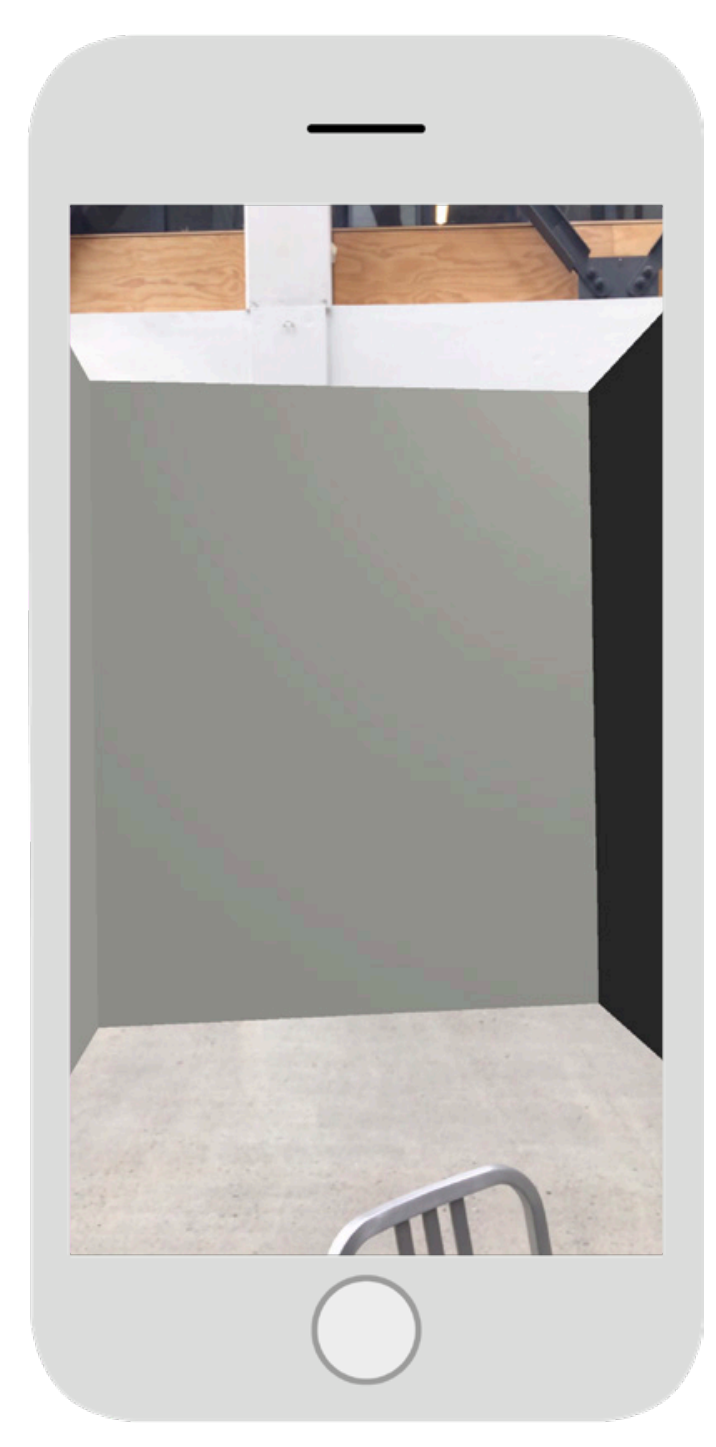

Figure 16: An attempt at walling in the viewer, suffered from inaccurate tracking 
Greyboxing is a strategy used in game design to map out scenes and levels with primitive shapes before creating the full assets for that scene. Using this technique allowed for a better understanding of the space and scale of different digital layouts when compared to the physical environment. As an initial exploration into shape and place, different layouts were used to explore what kind of scene could be added to the physical environment. Objects were laid out in a variety of ways, testing different layouts and types of scene designs. This included floating objects around the camera, objects spread around the ground, and large objects walling in the viewer. Instantiating things around the camera and using it as the anchor was interesting as it allowed the viewer to move and look back at the scene, however aligning things to the physical ground was difficult without first anchoring it down somehow. The physical location impacted the success of the scene so understanding the layout of the physical location would be important when designing more developed experiences. Adding floating objects to the scene worked well as there were less physical objects in this space.

Trying to build more large-scale environment assets (Figure 16) proved interesting and allowed for the potential to create a sense of enclosure or a full digital space within the physical scene. However as with the larger scale tests, the tracking did become weaker and the models shifted a small amount within the space.

Interactions in $\mathrm{AR}$ are important. $\mathrm{AR}$ is delivered through a highly interactable piece of hardware: the mobile smart phone. It was important to note that the user is familiar with tapping to interact with the device.
This interaction could then be used for anchoring content and interacting with that content.

AR does allow for a different way of interacting with the content, namely in a spatial way, allowing the user to move around the digital content and get close to it, as if it was a physical object. This is documented in figures on the next page.

The assets placed into AR are mostly limited by the physical location they are eventually placed in. However, there are still some aspects of 3D models for Mobile AR that should be considered, namely that the polycount and texture sizes should be kept low to guarantee smooth performance. If the digital assets started lagging and jittering it would create a disconnect with the physical environment. This disconnection results in a less polished experience. It could also lag the device, resulting in less accurate tracking, affecting the experience as a whole.

The lighting of the digital content within AR should imitate the lighting of the physical space it aligns with. This is to strengthen the connection between the digital content and the physical environment. Creating content for specific locations allows for lights to be specifically designed with this in mind, placing and angling the lighting in relation to light sources in the physical environment. 


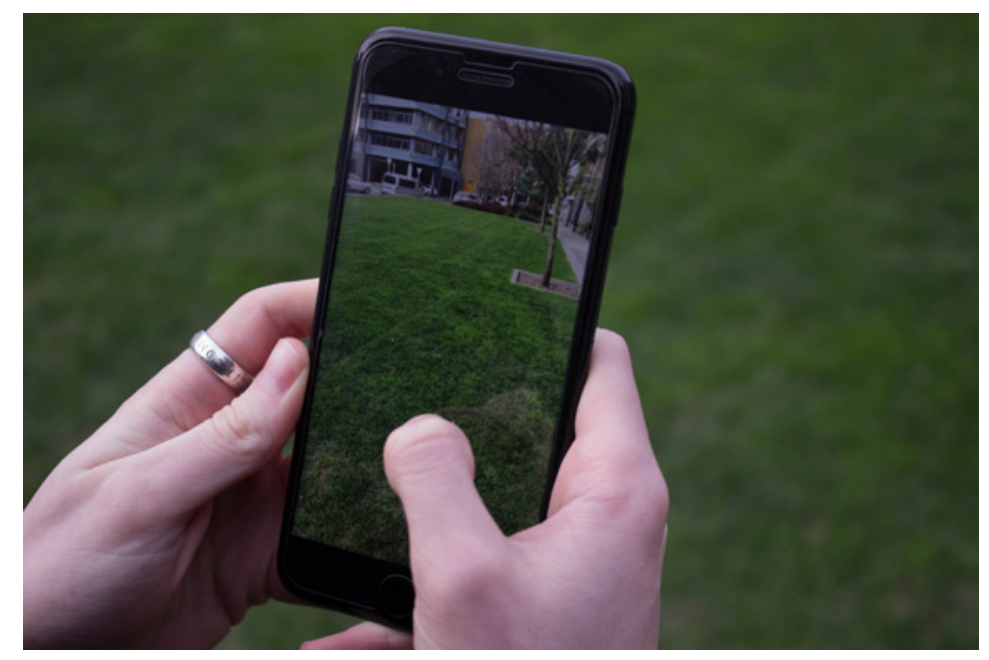

Figure 17: View through the device with no digital content

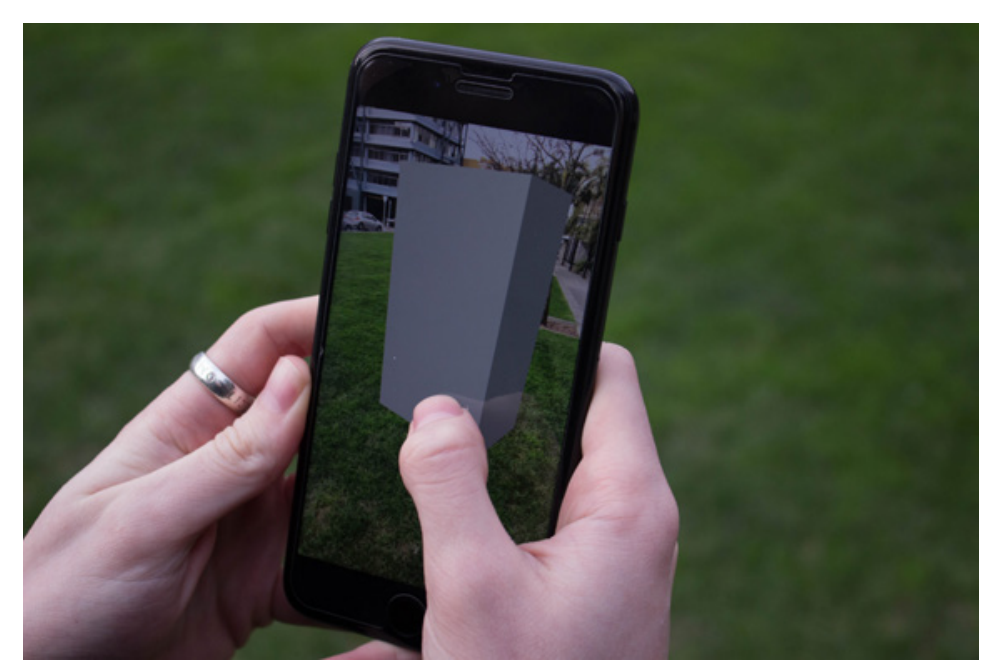

Figure 18: Content placed with a tap targetted at the ground

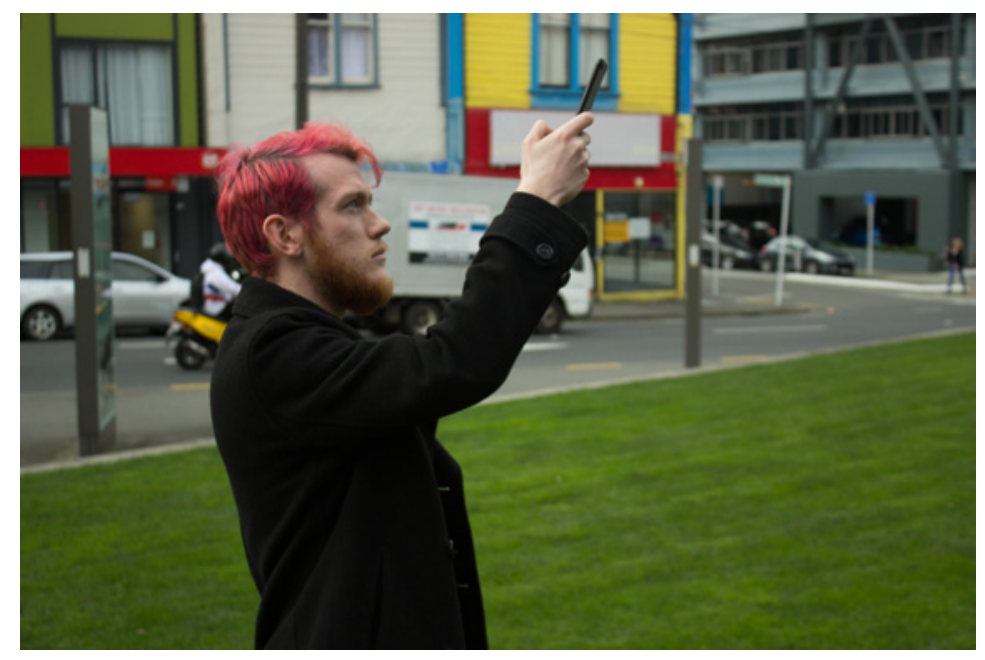

Figure 19: Viewing digital content through a higher angle

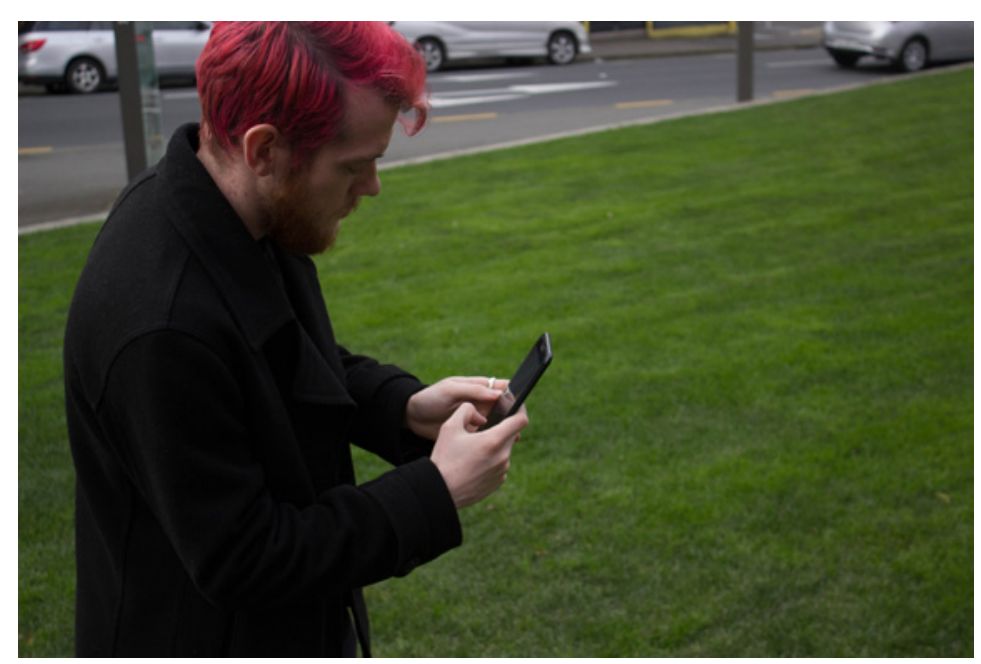

Figure 20: Moving physically to focus on details in digital content 


\section{Location and Content Exploration}

As previously stated, Digital Street Art should remain Street Art, using the street as an artistic resource and engaging with the public space (Riggle, 2010). Therefore, to create Digital Street Art in Wellington, the streets of Wellington need to be explored and understood, both physically and contextually. Wellington's rich body of traditional Street Art was also explored during this phase of research, to potentially inspire stylistic or thematic influence.

This section explores Wellington for potential sites of Digital Street Art. This allowed for a better understanding of how it could be used as an artistic resource. The focus of this exploration was to test both the technical feasibility of various locations in Wellington, and to scout locations that could be used to give context to Digital Street Art. This exploration was important as it allowed for an understanding of the contexts and locations that could be used as a resource for the developed designs in Chapter 4, so that they could better engage with the public space and provoke a response from viewers.

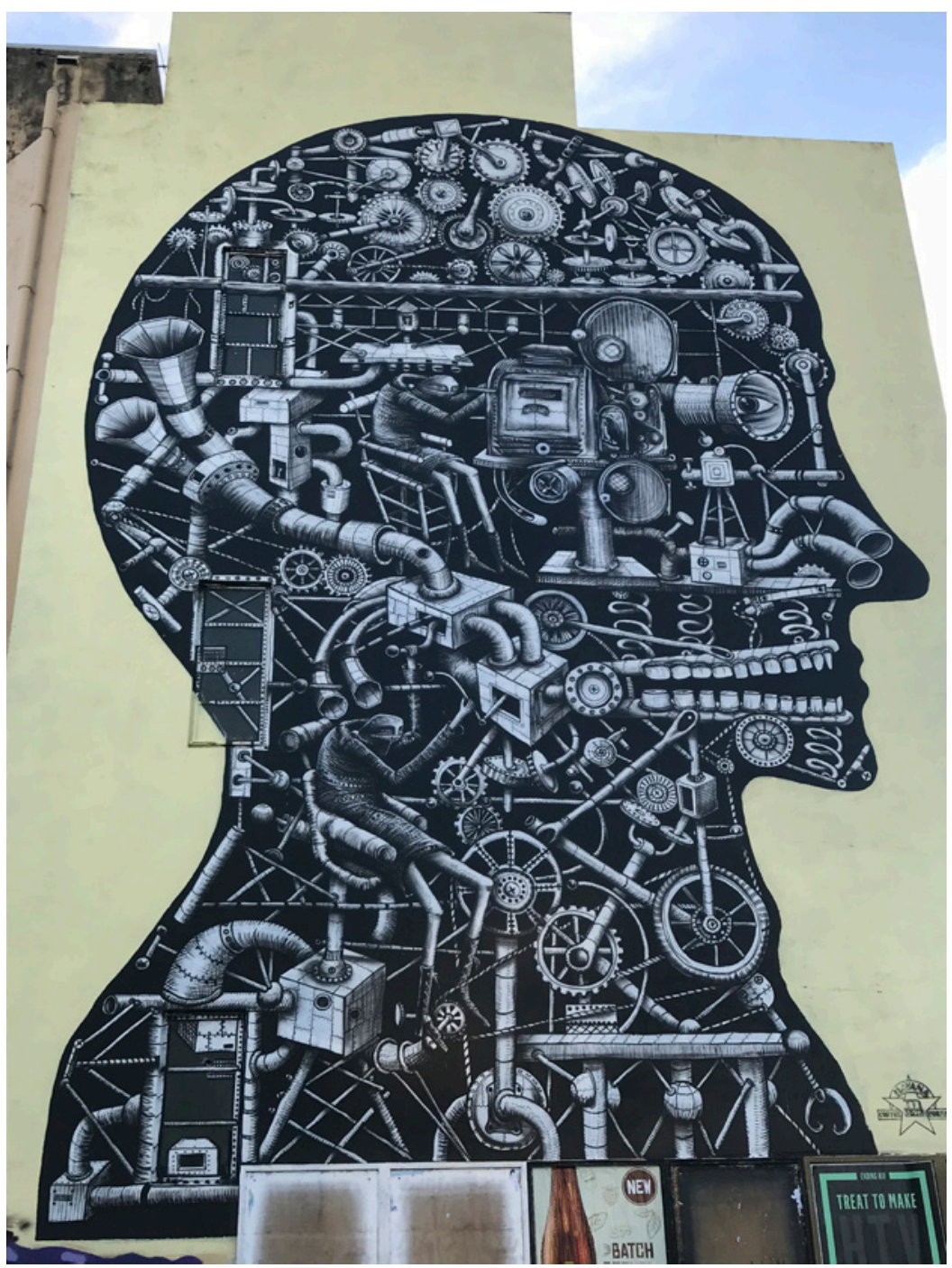

Figure 21: Large Scale Street Art Piece commenting on Wellington's love of coffee 


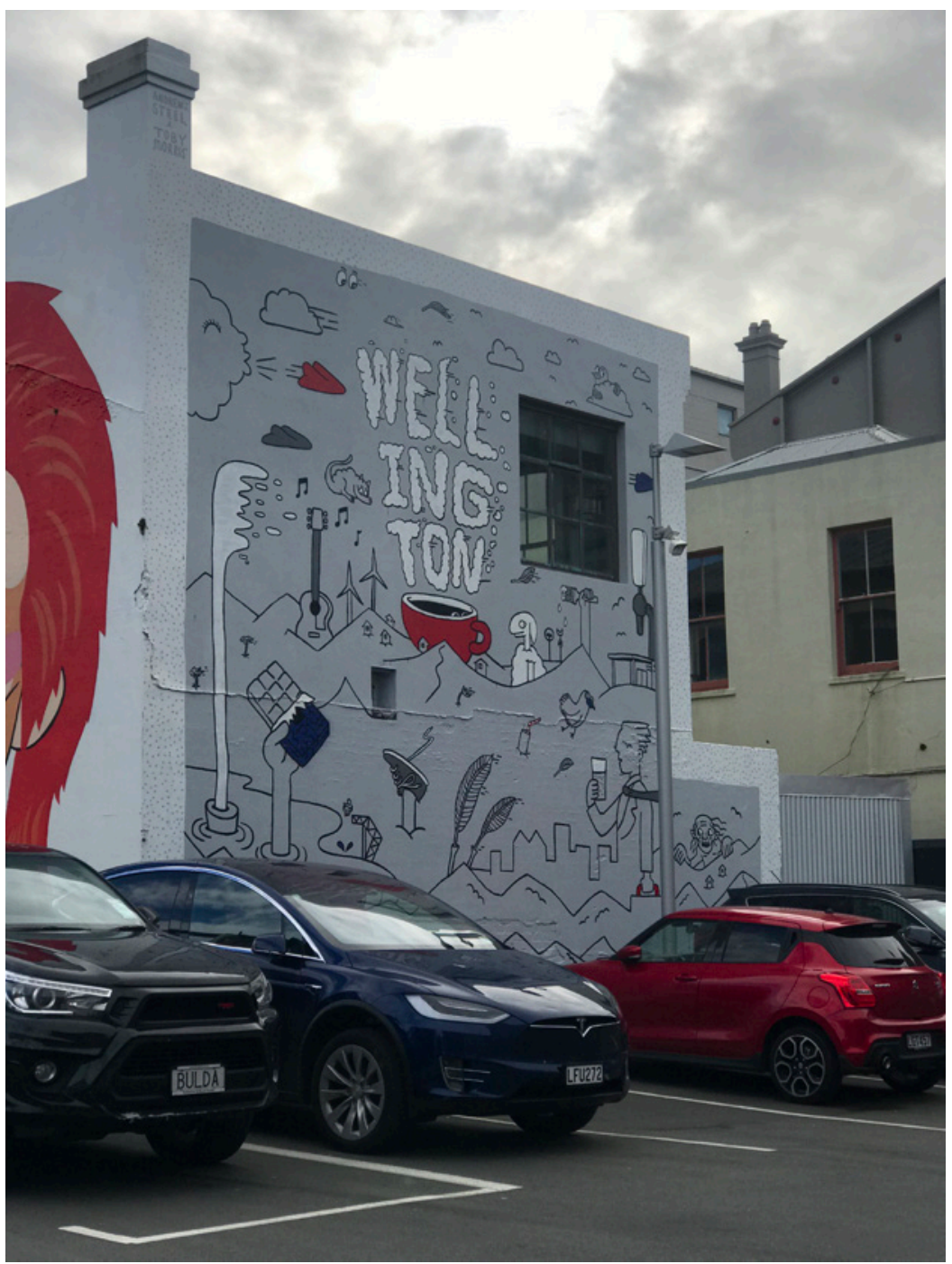

Figure 22: Street Art piece referencing many stereotypes and landmarks

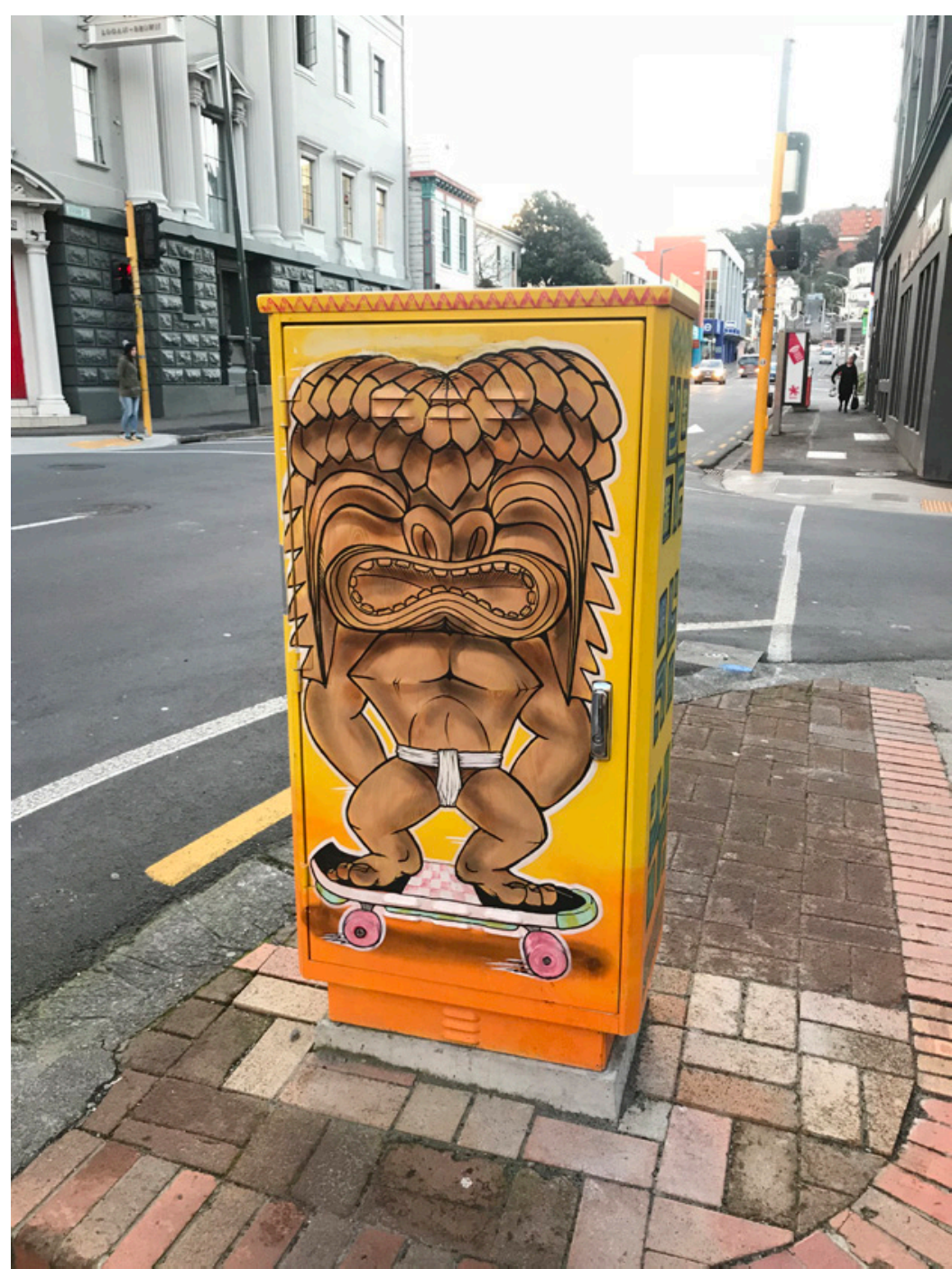

Figure 23: Skateboarding character on a power box on Cuba St, where skateboarders are known to skate down the centre of the road 
Figure 24: BMD for anti-shark finning, Wellington, 2013 


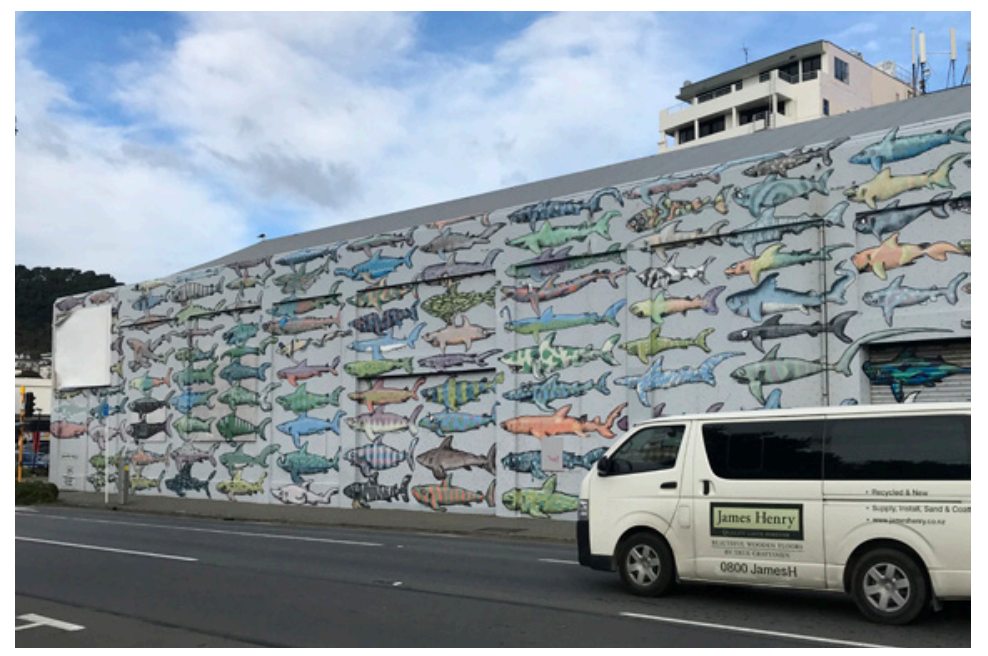

Figure 25: BMD's shark wall referenced the fact that shark finning was still legal in New Zealand as of 2013 (it has since been made illegal)

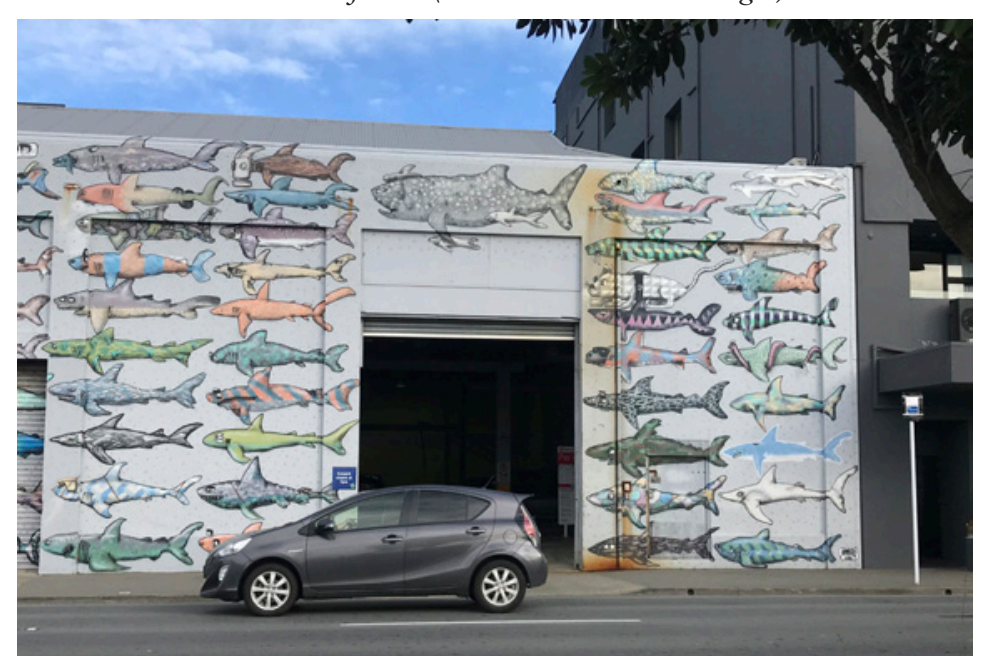

Figure 26: The shark wall has since become a local landmark

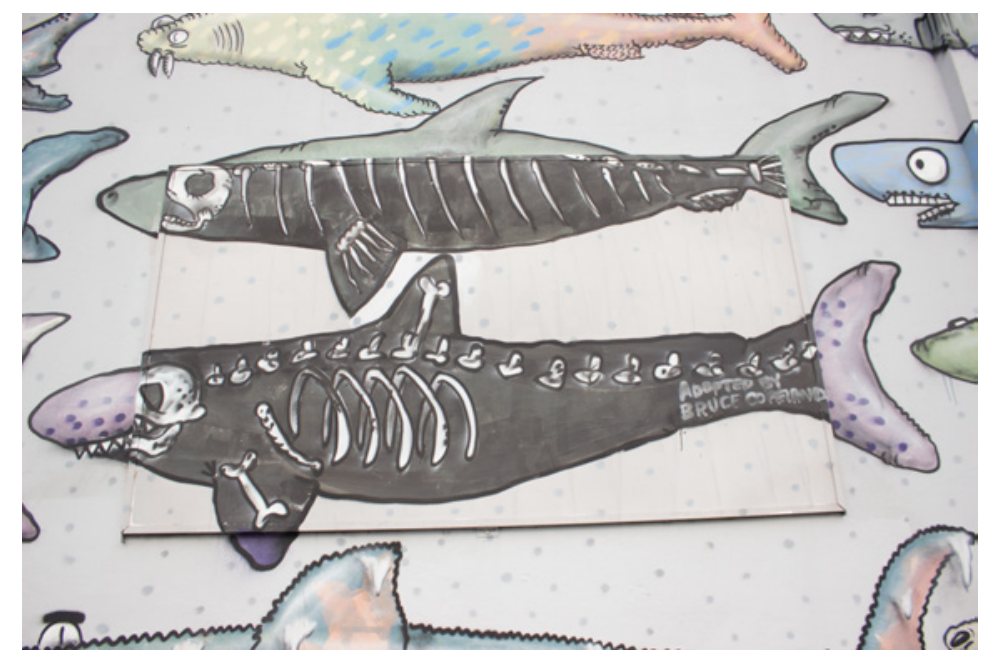

Figure 27: Shark on the shark wall

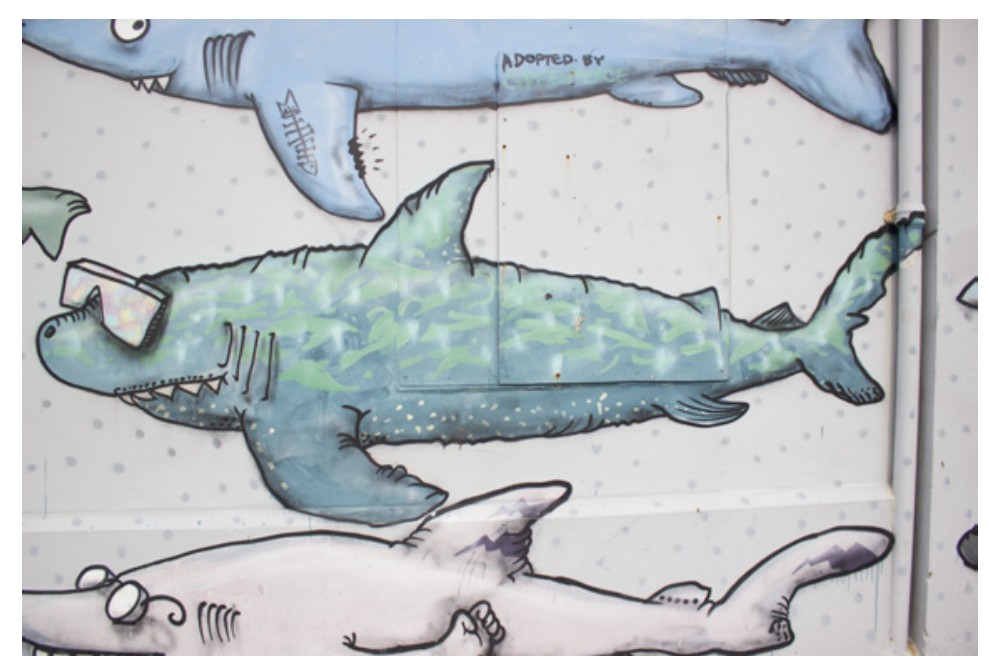

Figure 28: Shark on the shark wall 


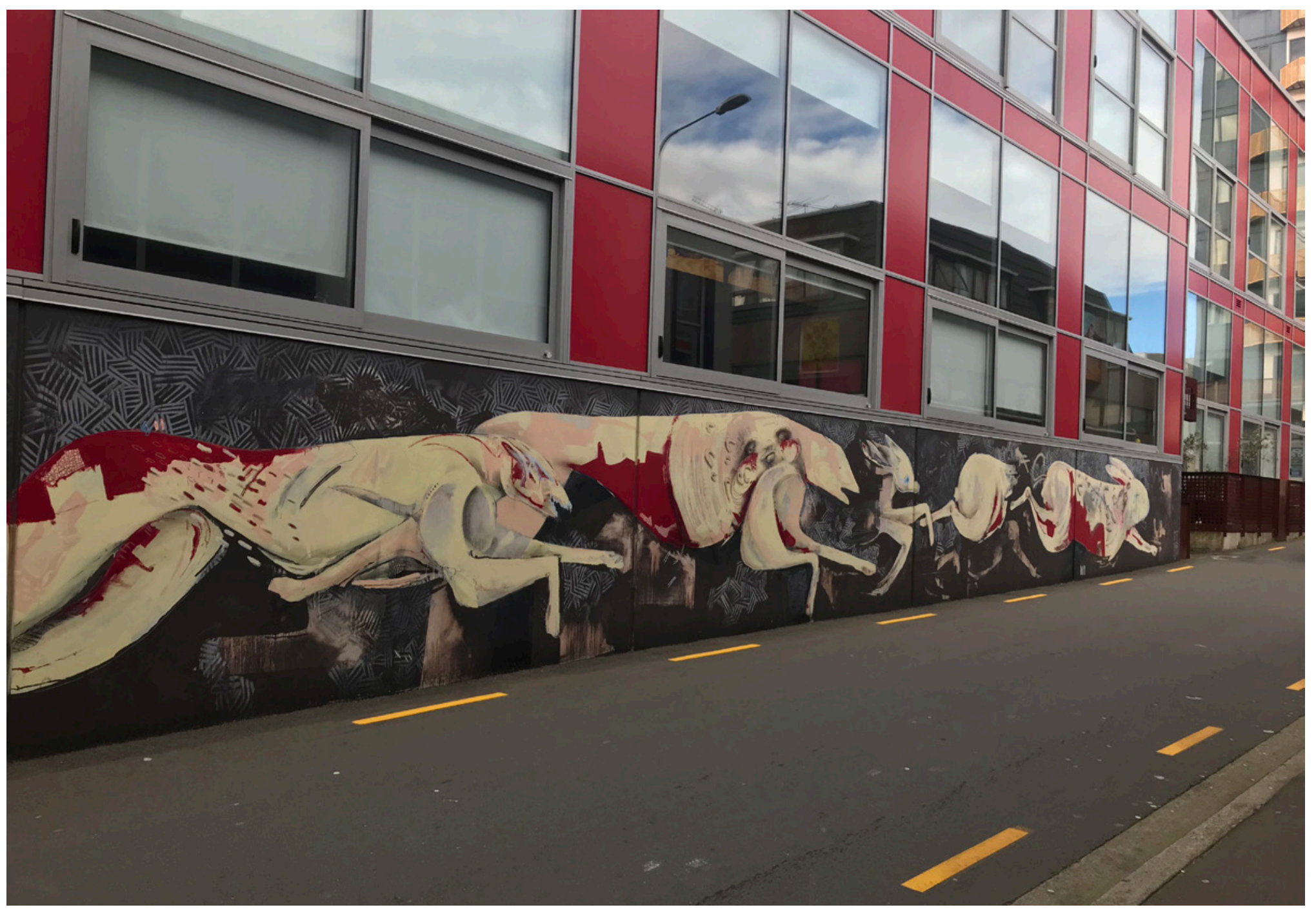

Figure 29: dashing animals along a low wall 


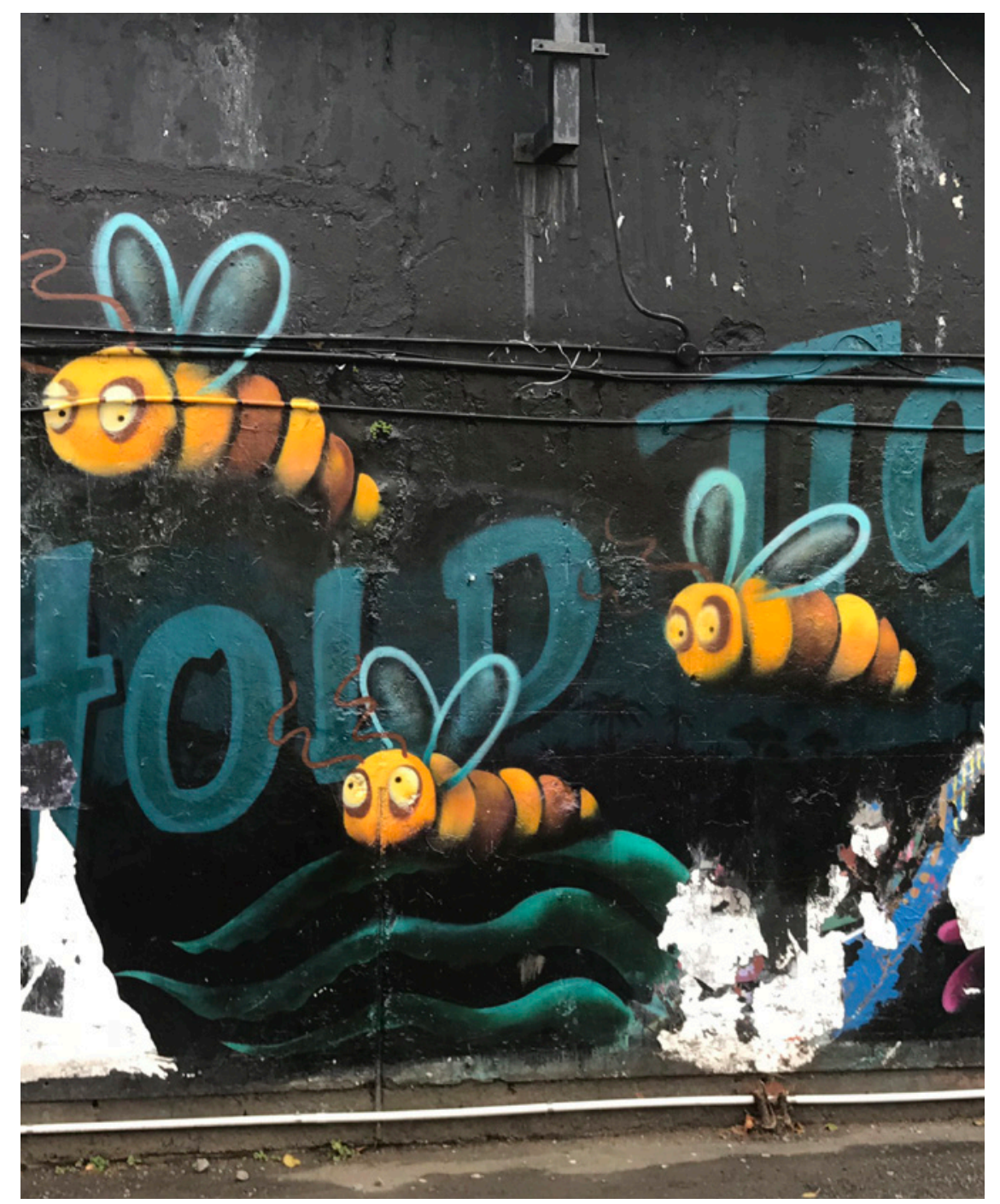

Figure 30: Bees on Cuba Street

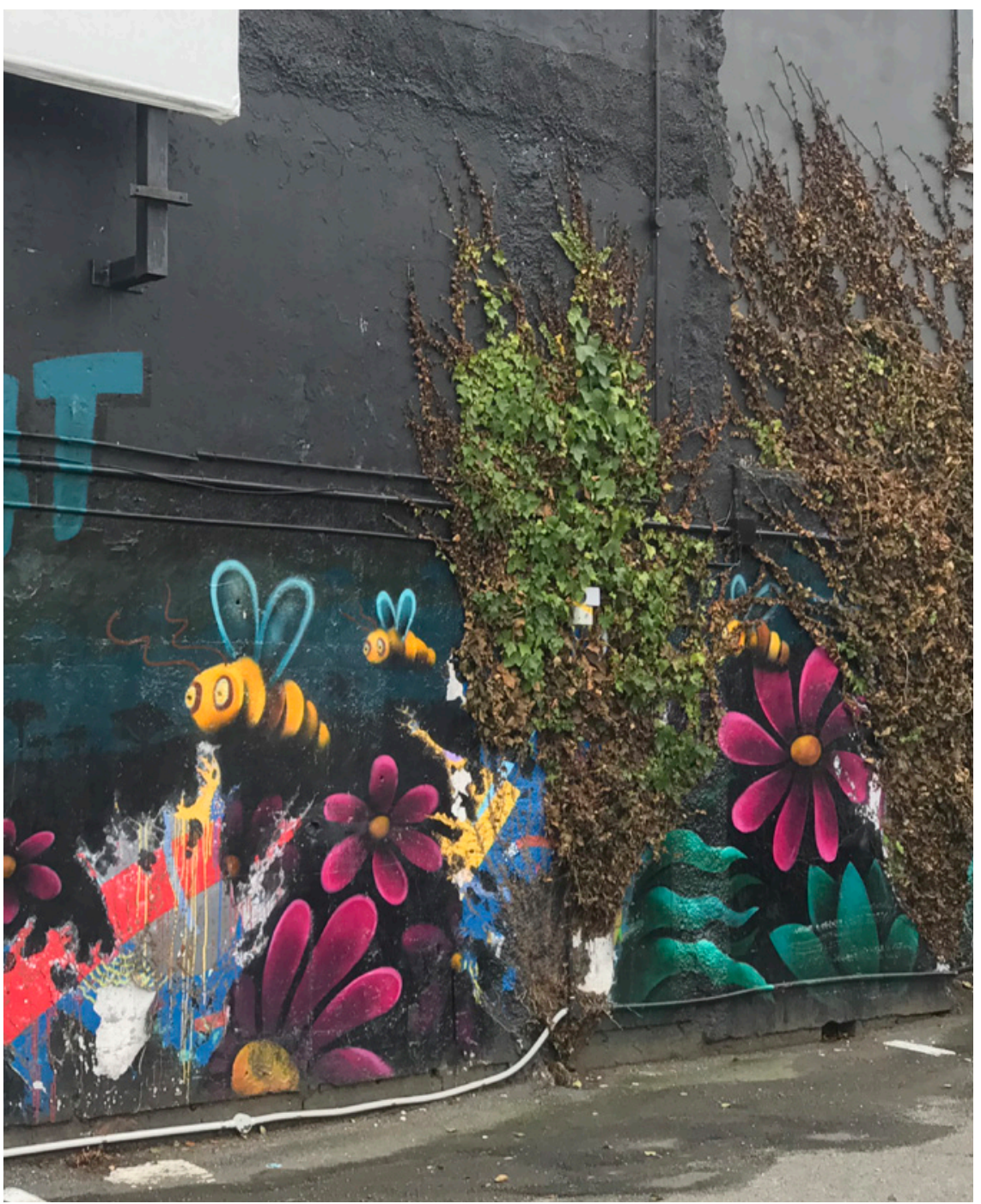

Figure 31: Street Art making use of the vegetation 


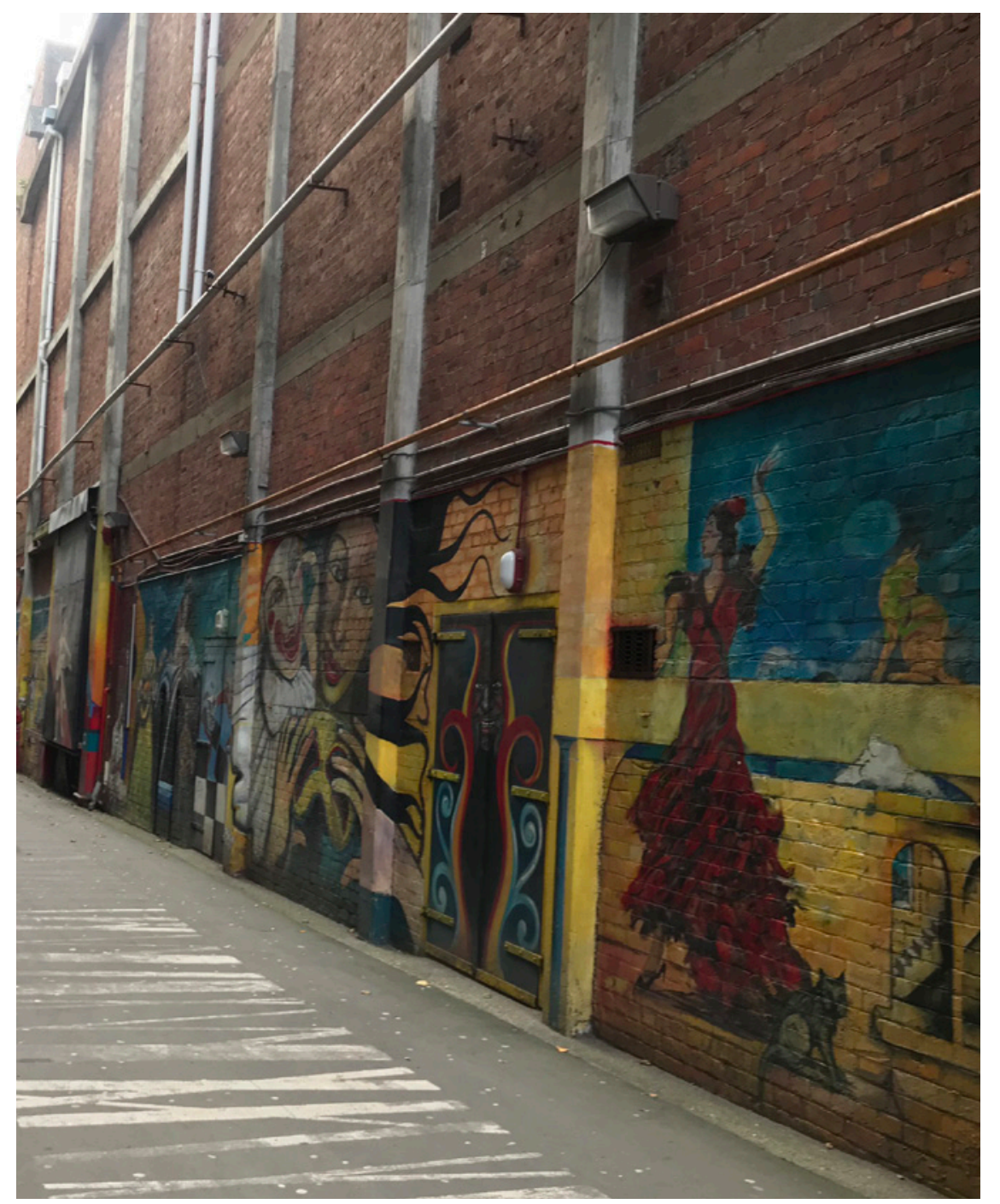

Figure 32: The alley by the opera bouse is packed with street art referencing the performing arts.

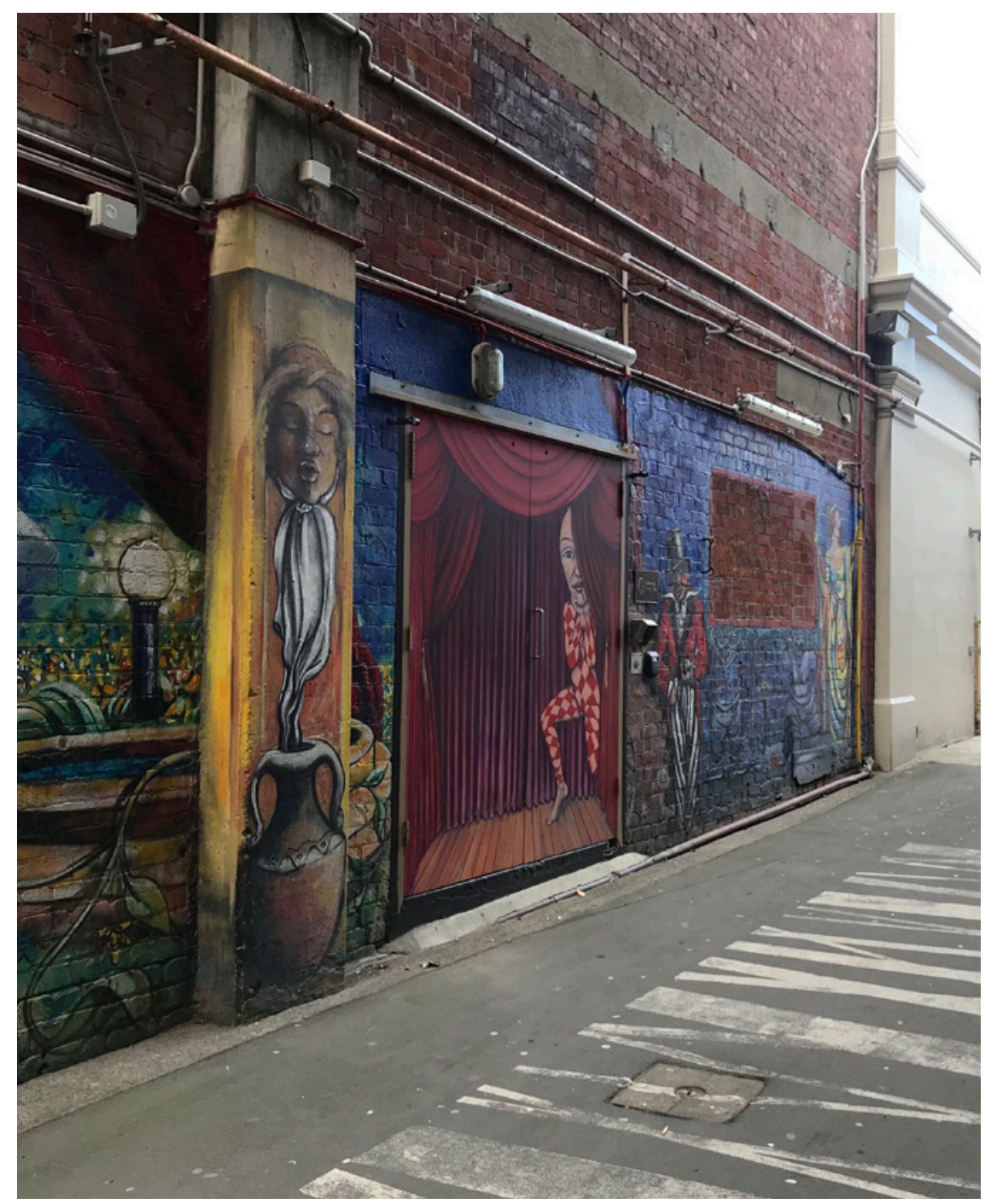

Figure 33: Different angle of the opera house alley 


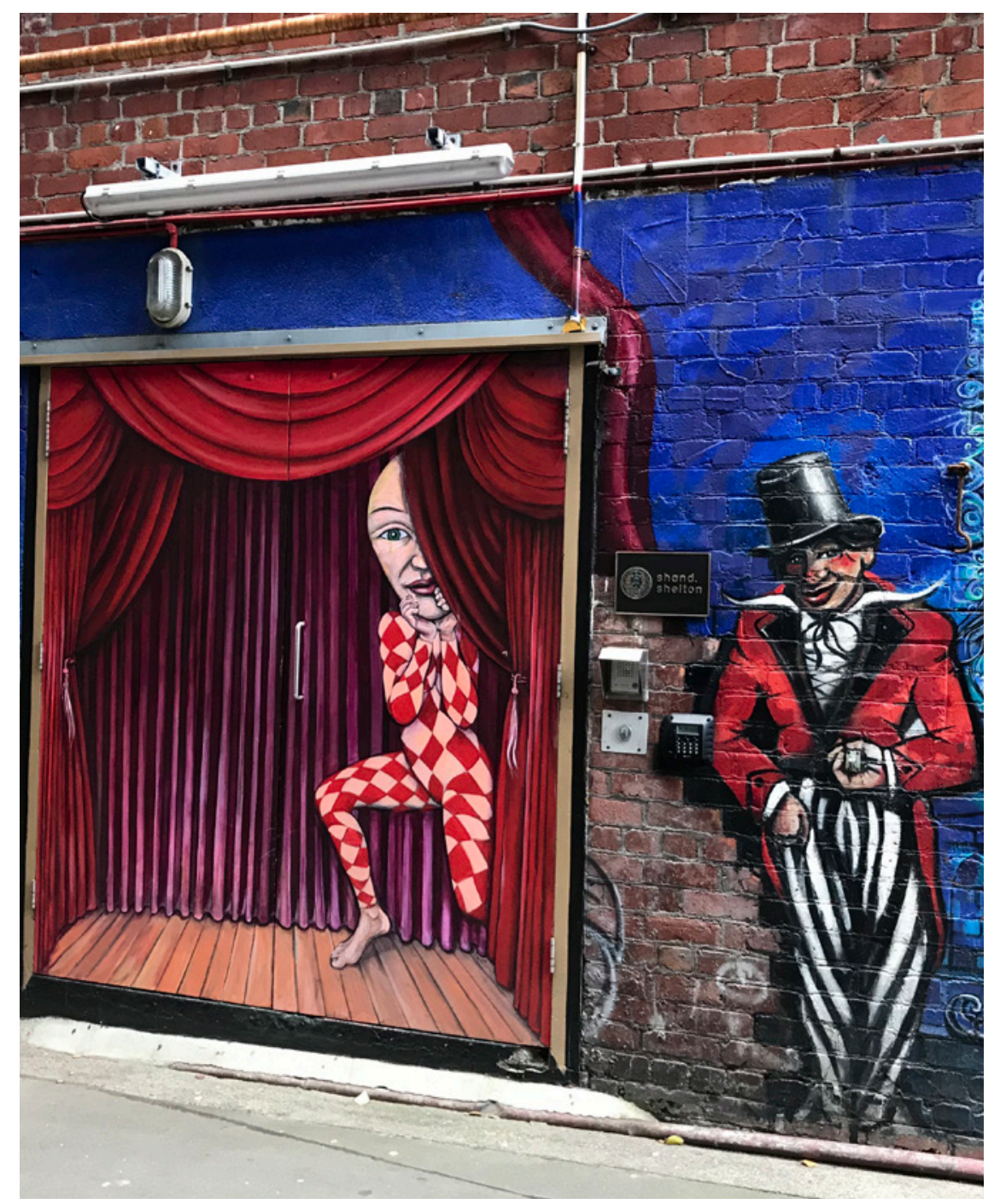

Figure 34: Example piece of the mural

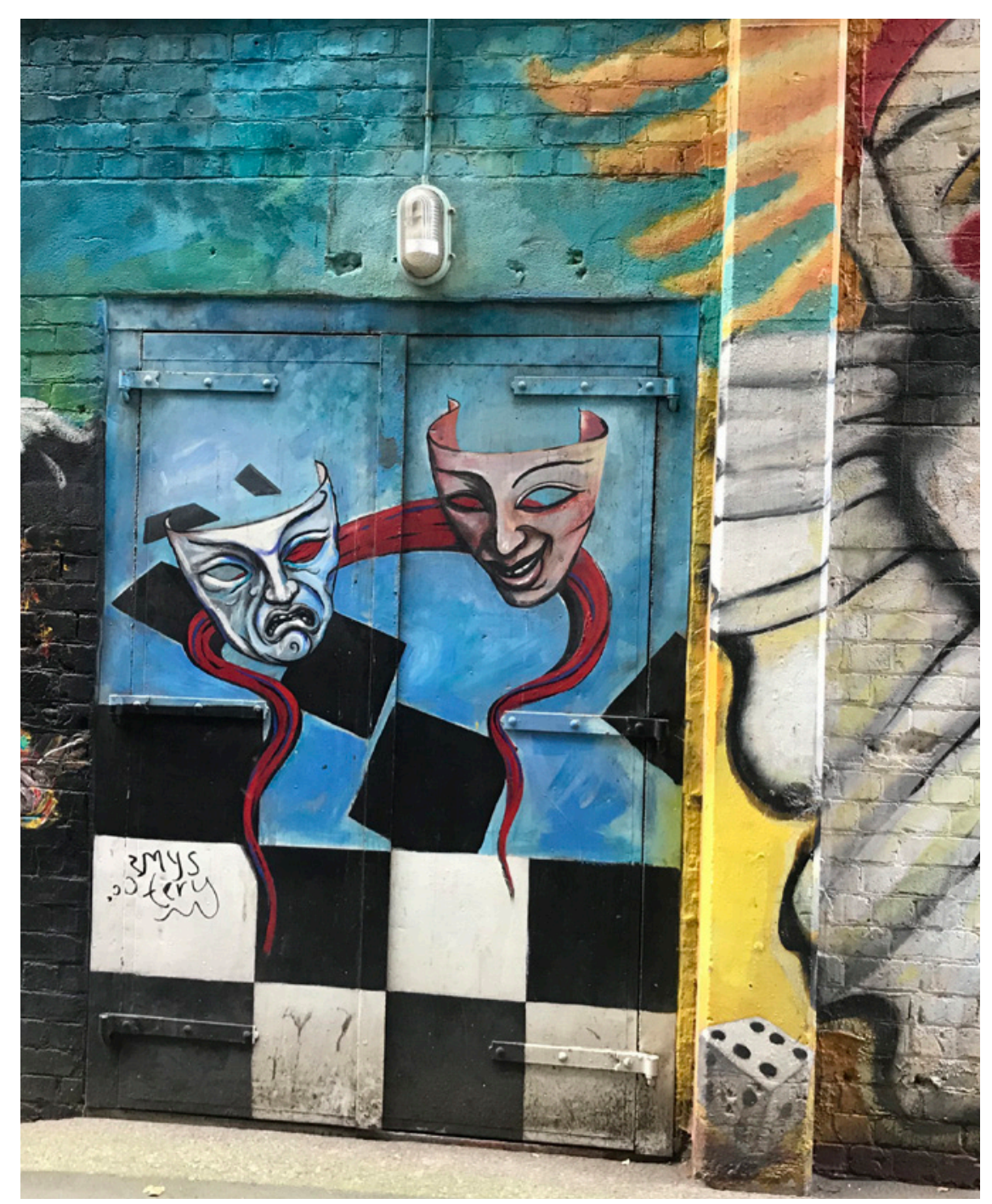

Figure 35: Painted door along the mural 


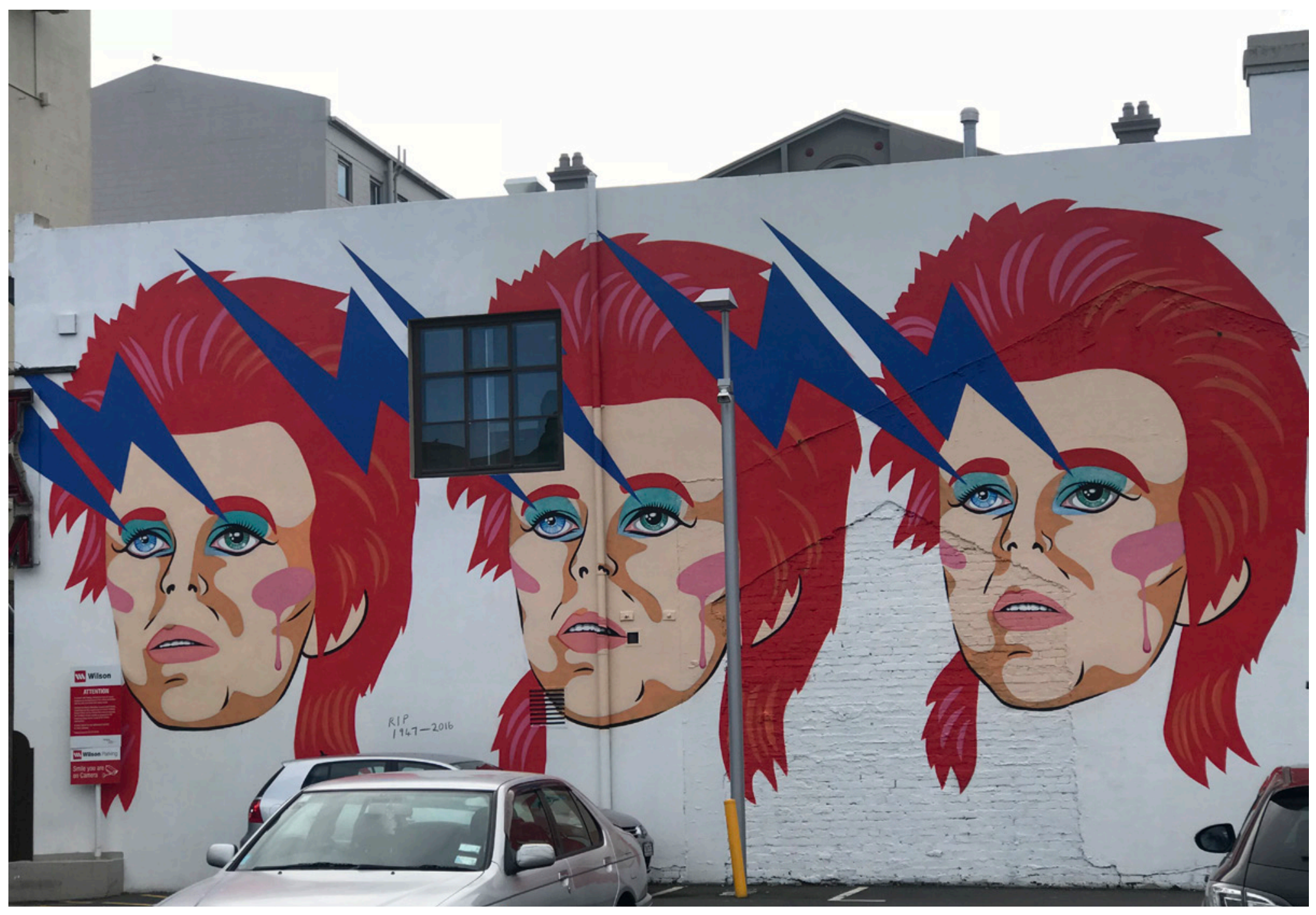

Figure 36: A tribute to David Bowie appeared after he died 


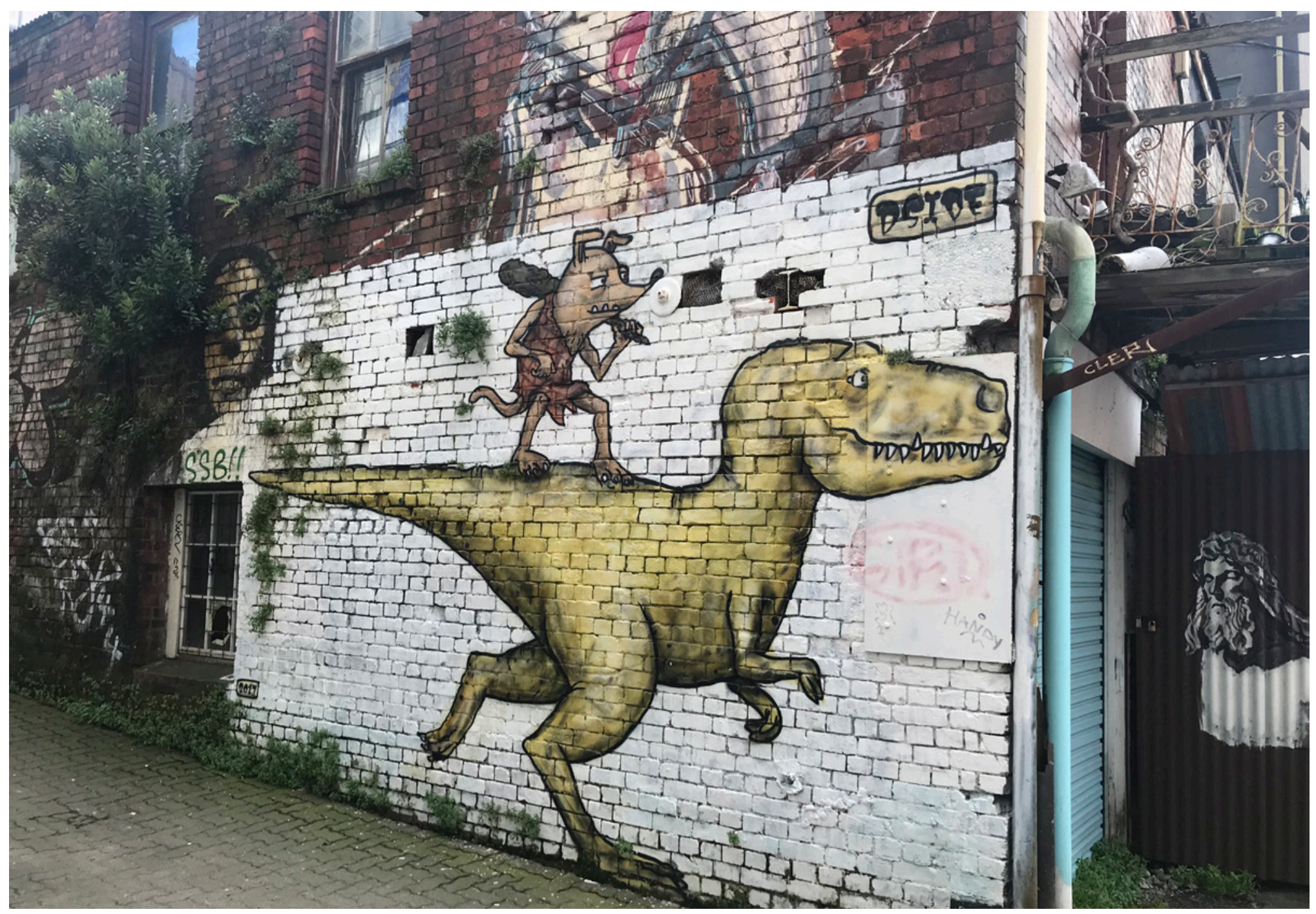

Figure 37: A dinosaur on an old building 
Wellington is a centre for urban creativity, both sanctioned and unsanctioned. These street art works responded to the urban environment, and addressed interesting histories, topics of concern and raised awareness with their art.

Wellington has many locations that could serve as inspiration for Digital Street Art. These were then examined with respect to adding simple AR content, both to test tracking stability and suitability for further exploration of content creation.

Popular Wellington landmarks were considered as sites for AR Street Art, as were places with high foot traffic. Other places considered were locations that could potentially lead to a theme or topic for designed works of Digital Street Art. This included the Waterfront, CBD,

Parliament, Cuba Street and outside the Victoria University of Wellington campus.

Testing tracking and plane generation at many locations showed that most street and sidewalk surfaces offered stable tracking due to their rough textures, and that there were also options for applying content to raised surfaces and vertical walls in many locations. On the following pages, various tests of tracking accuracy and suitability are documented.

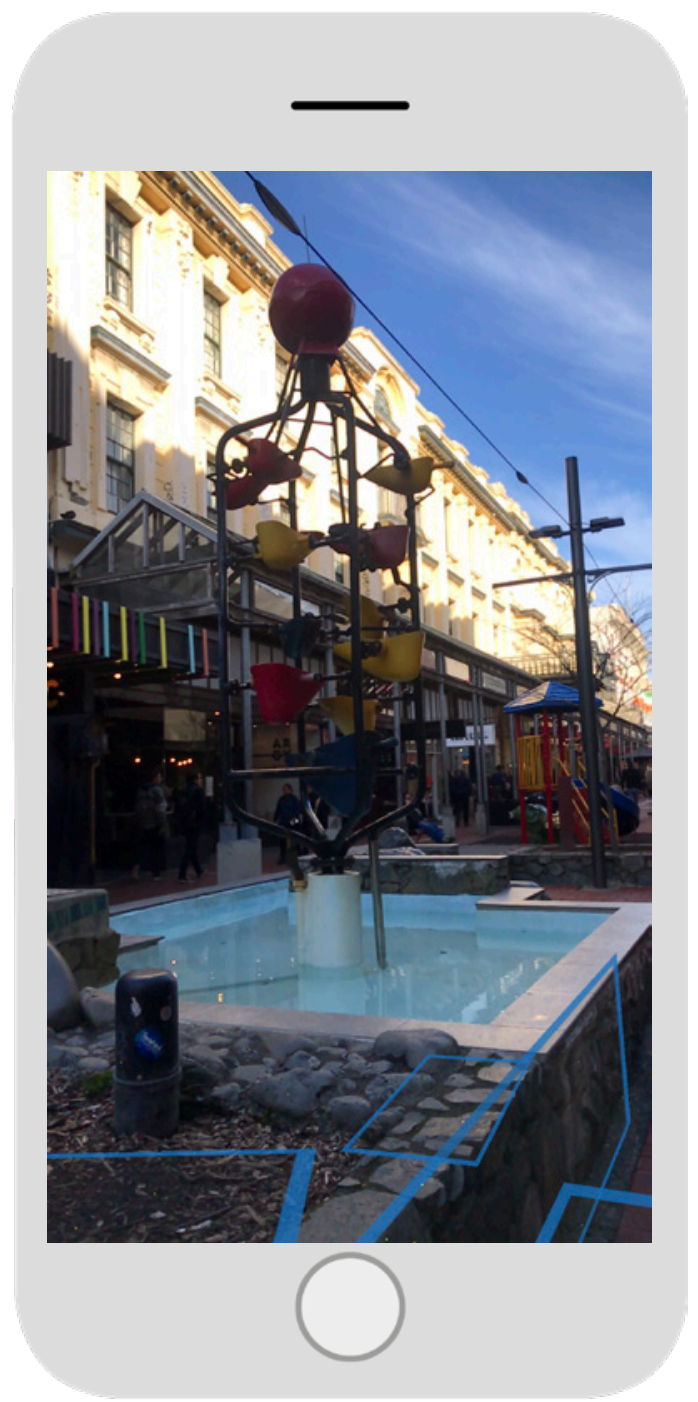

Figure 38: Tracking and Generating planes around the Bucket Fountain in Wellington 


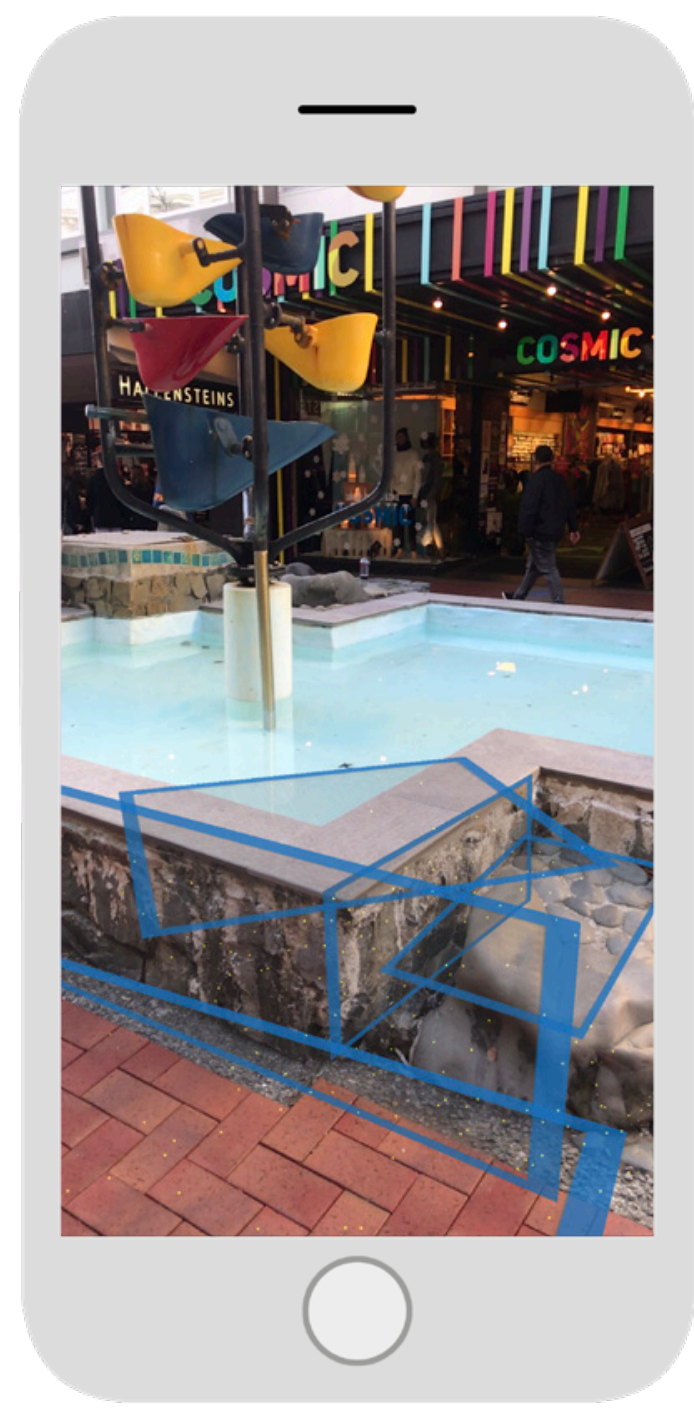

Figure 39: Several areas around the fountain generated planes

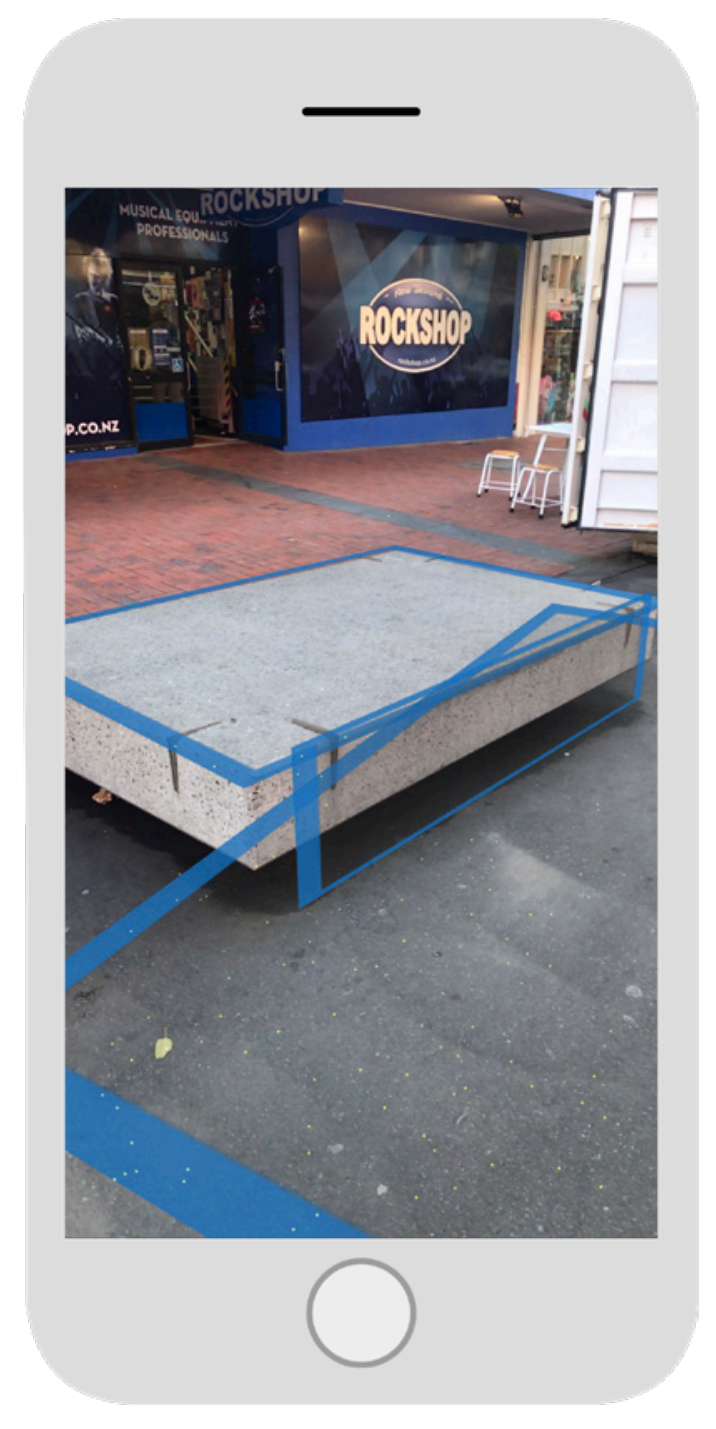

Figure 40: Tracking on Cuba St, both the road and raised bench

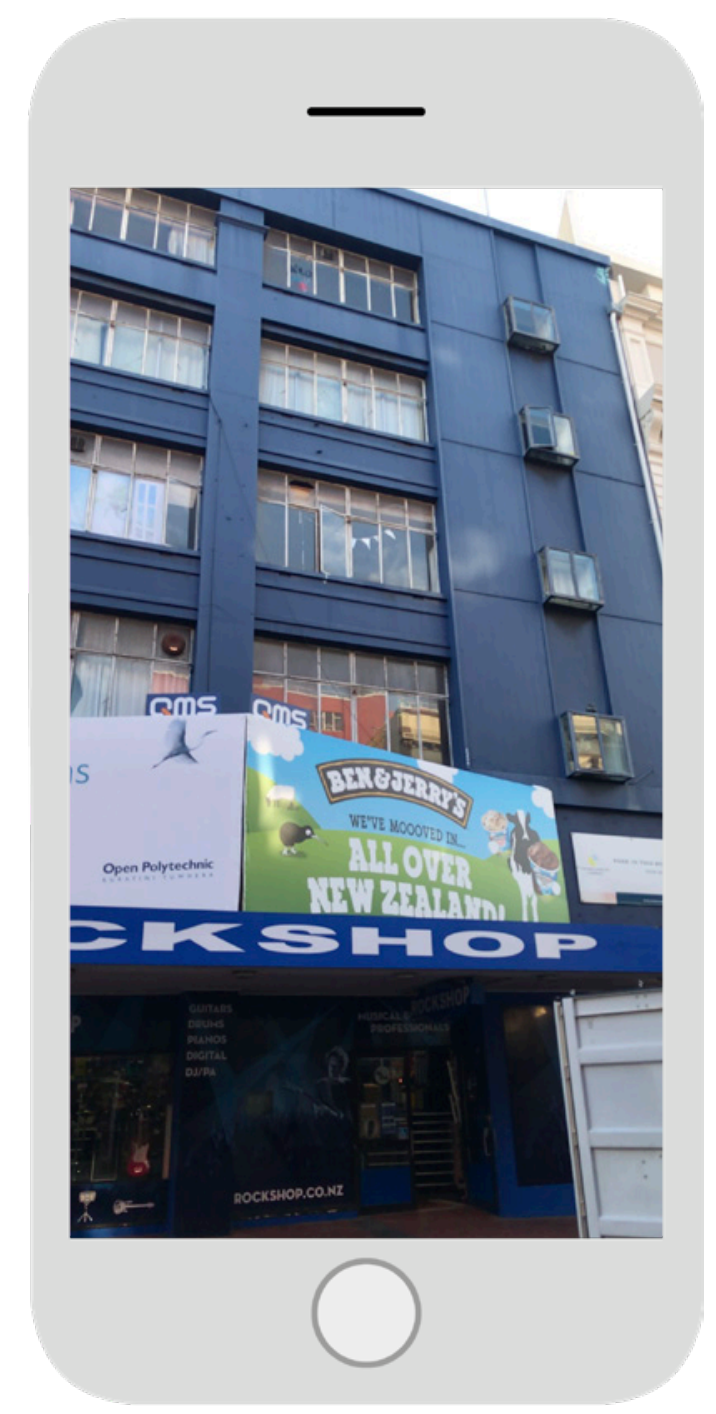

Figure 41 : Flats on Cuba street above figure 40, earthquake prone but still inhabited 


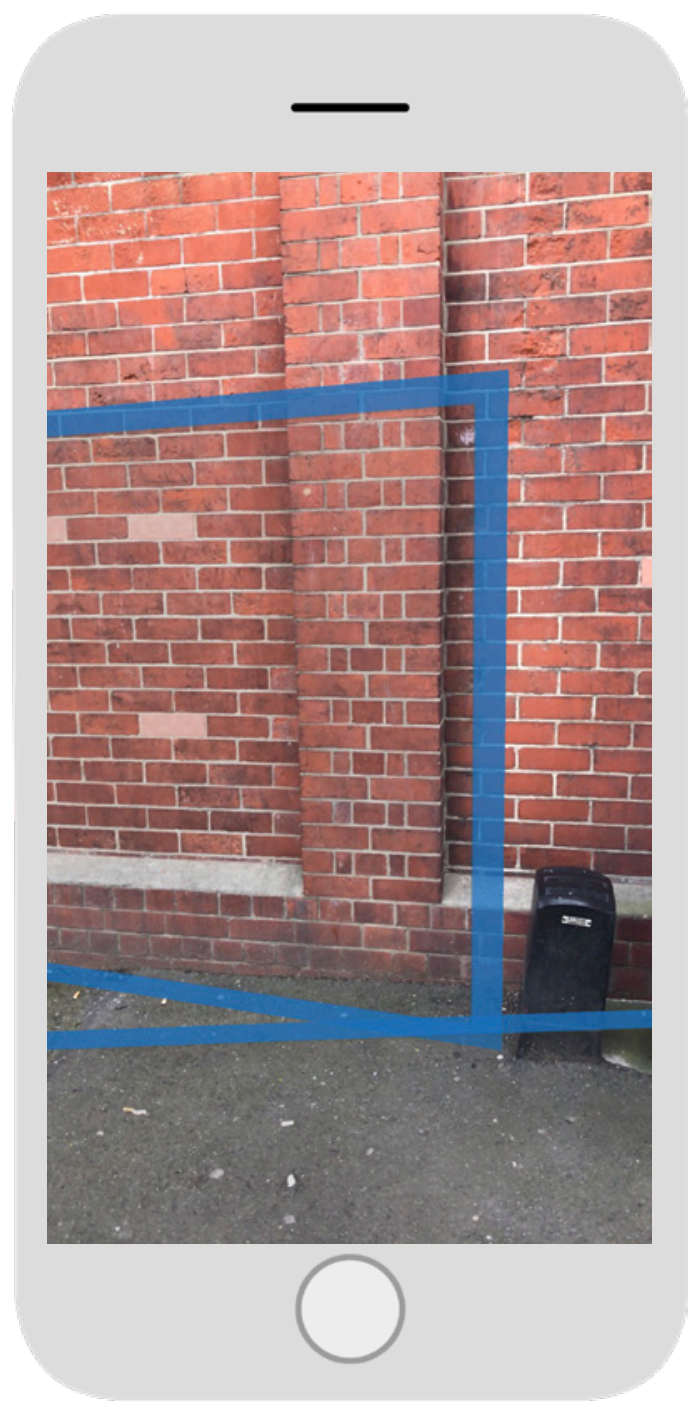

Figure 42: Generating horizontal and vertical planes along the sidewalk

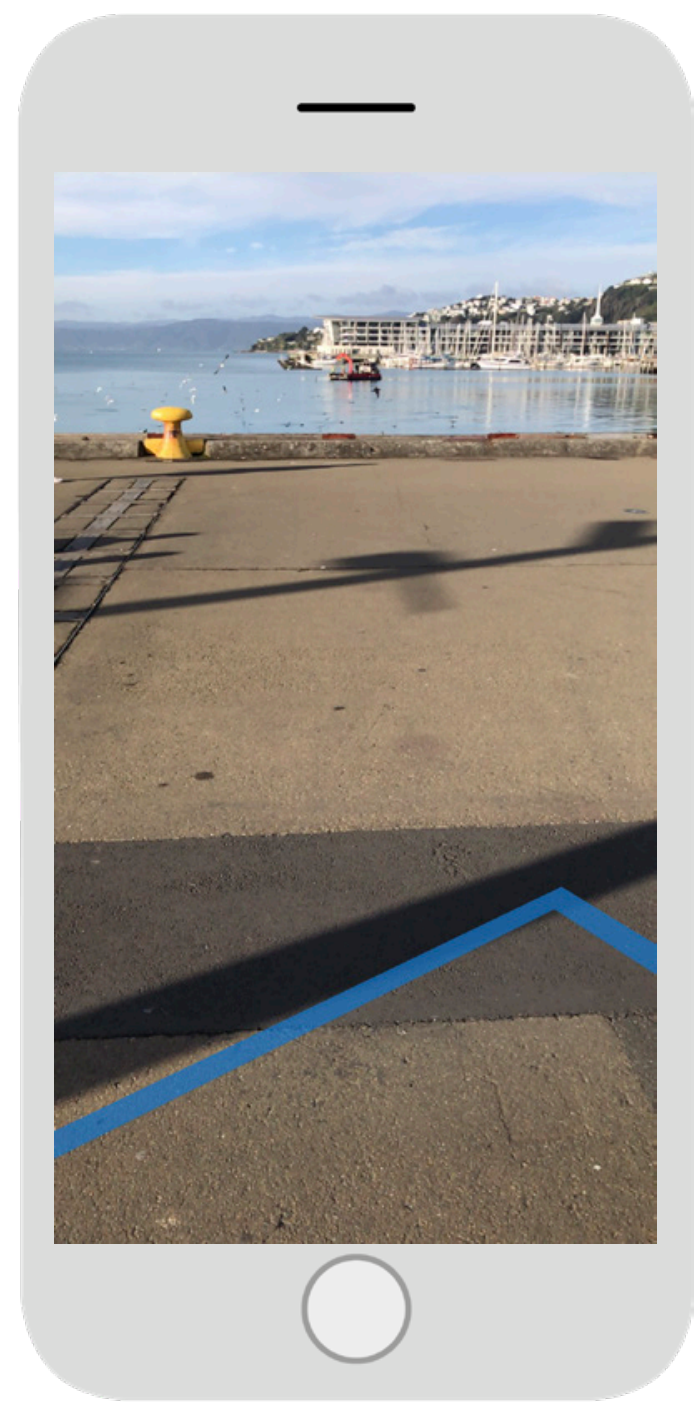

Figure 43: The waterfront area offered wide spaces for tracking

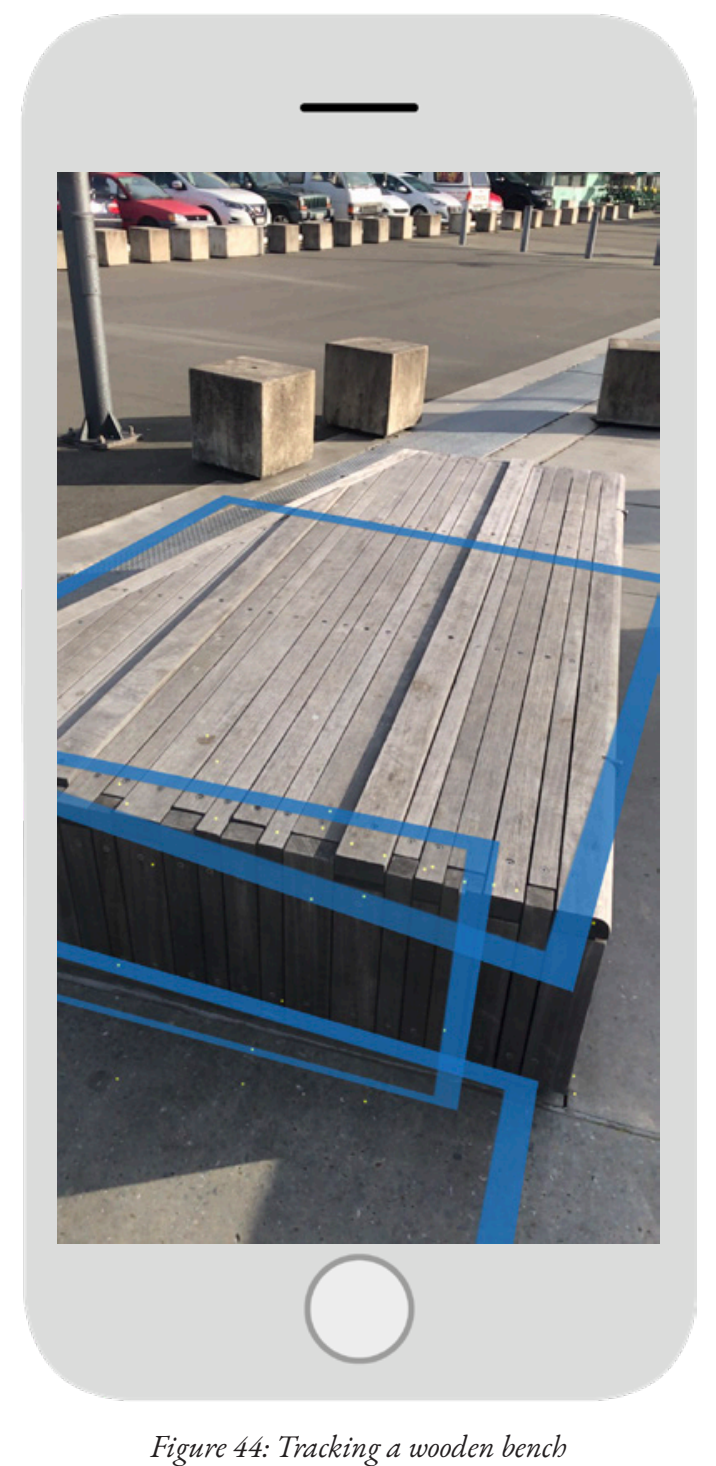

Figure 44: Tracking a wooden bench 


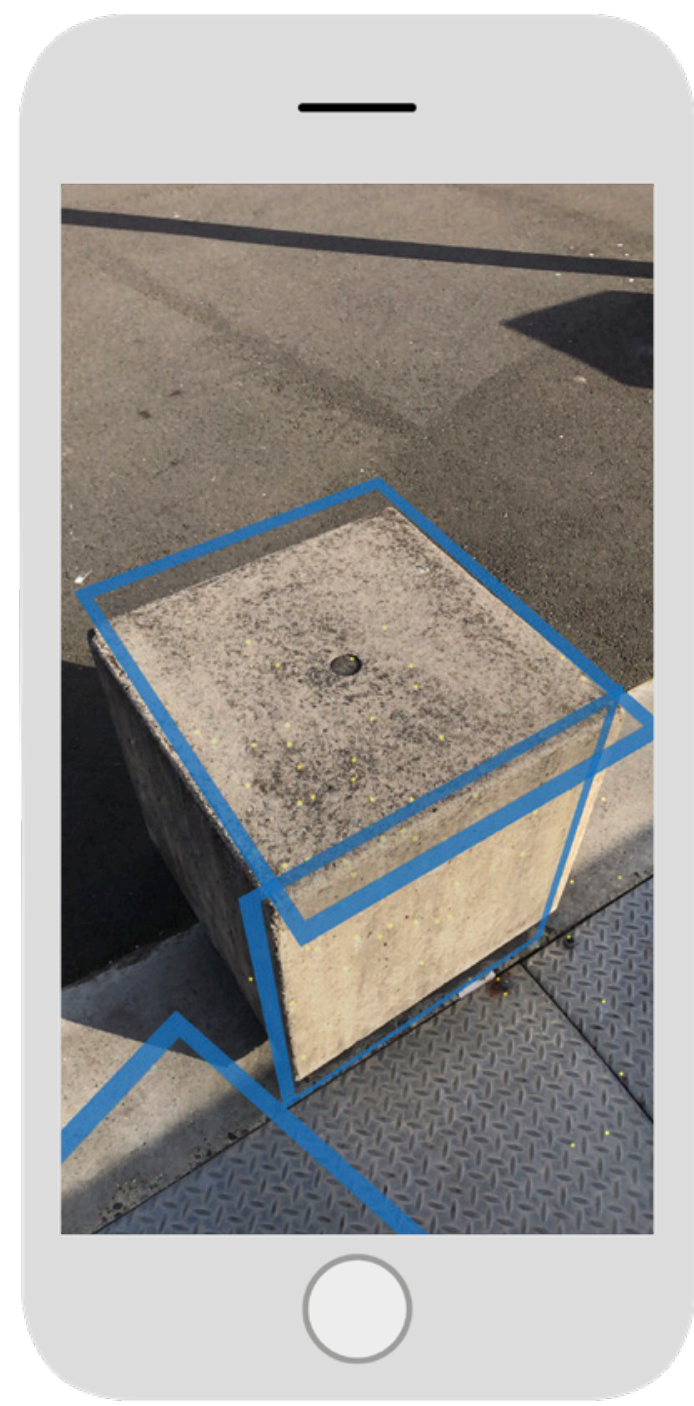

Figure 45: A small platform that could be used a podium for digital content

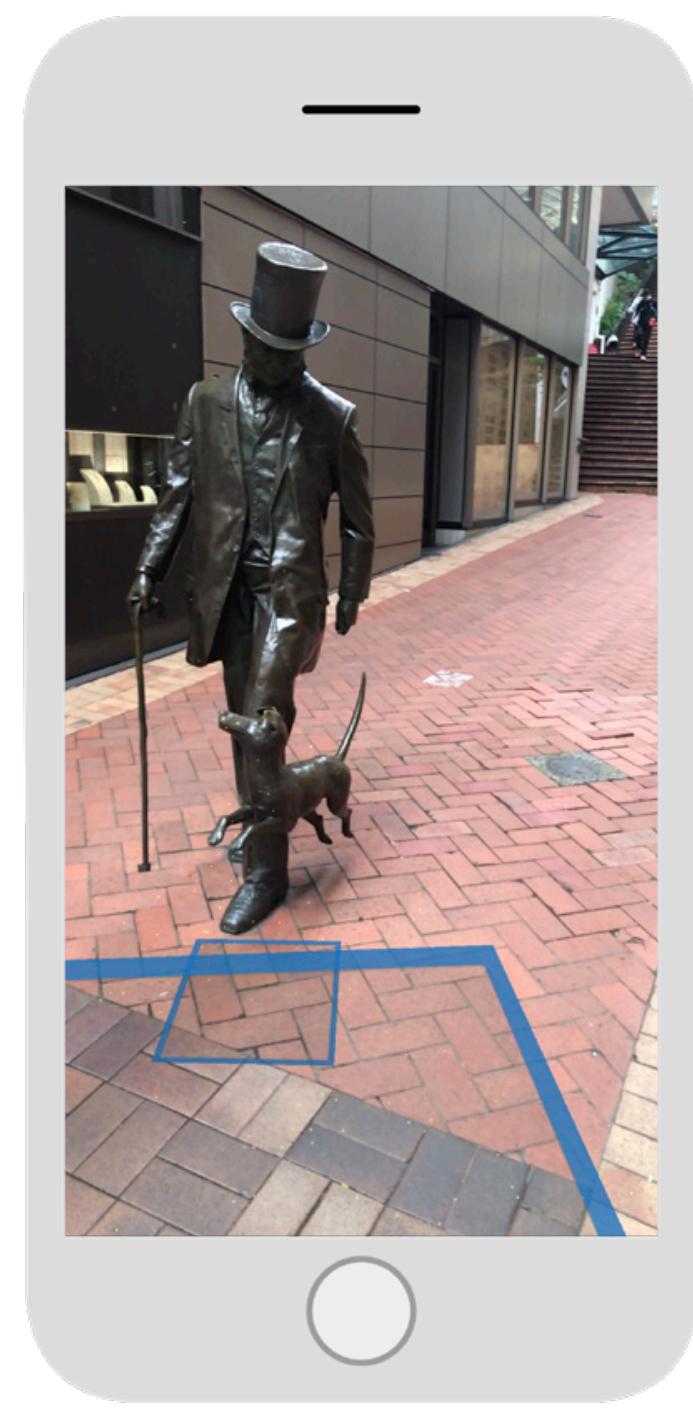

Figure 46: The brick sidewalks in the CBD tracked and generated planes accurately

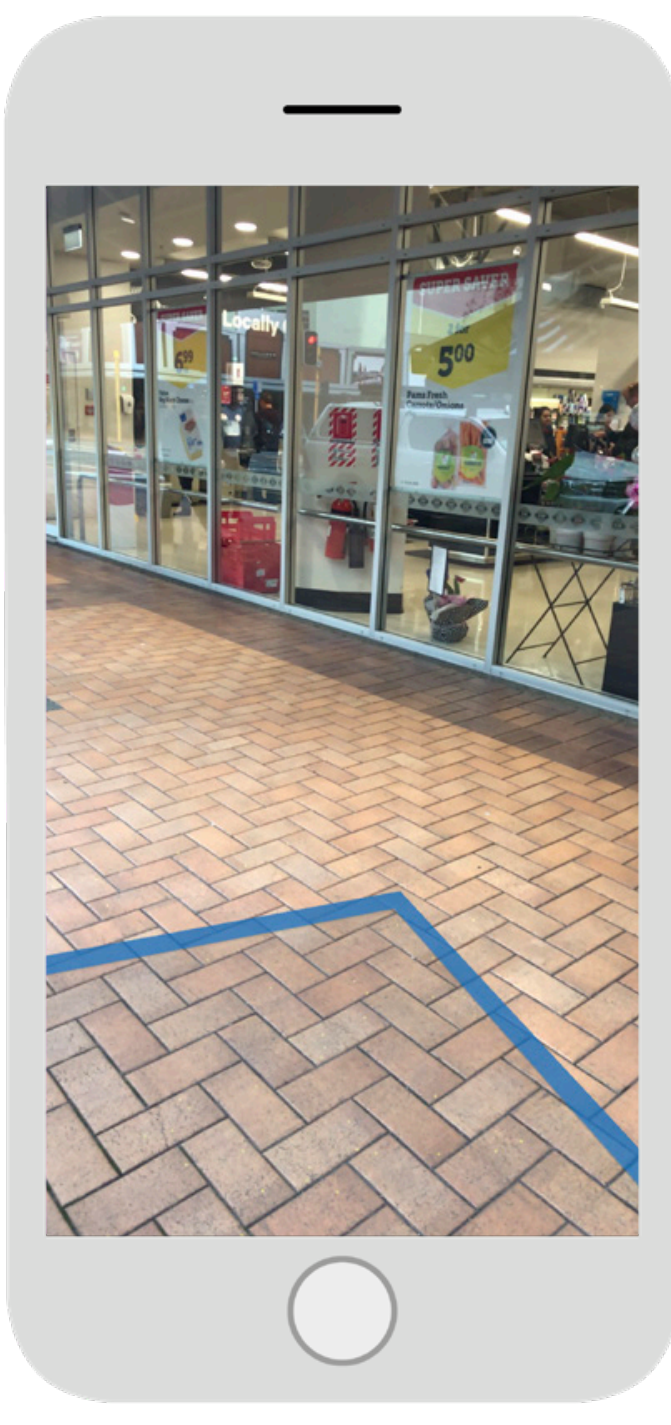

Figure 47: Sidewalk tracked well despite the presence of pedestrians 


\section{Findings}

This chapter was focused on situating an understanding of the technology and practical understanding of the research being conducted. First it explored artistic $\mathrm{AR}$ content for precedents and implications for functionality. Available tools for development were then surveyed and the technological constraints were determined, primarily that the remaining development in this research would be carried out on an iPhone 7+ using the Apple ARKit Software Development Kit (Apple Inc., 2018a) .

Building on this foundation, functional experiments investigated the functionality of the technology in more detail. Experiments proved that rough surfaces tracked accurately and consistently than smooth surfaces. This was also true for horizontal planes over vertical planes. Primitive digital shapes such as cubes were used to test how the scene would look with added digital content, allowing for an initial understanding of the bounds for content creation within the AR space the research was being conducted for. This showed that the AR technology allowed for most content specifications, although the larger the shape in any axes, the less stable the tracking resulting in the content shifting in its physical space. Any model that overflowed from a generated digital plane ran the risk of breaking, clipping through physical objects and causing a disconnect between the digital content and the physical environment.
The physical environment chosen was proven to be a fundamental decision for content creation, as different locations would allow for different content and apply different constraints. Placing objects within the research office required them to be very small to fit within a busy physical space but placing content within an open atrium space allowed for larger scale content, and more content in general. This was due to the amount of flat space available to track and generate digital planes. Greyboxing any final experiences would therefore be an important step before beginning asset creation and full development. Using this method, one could test the scales and shapes of the desired content in physical spaces and adapt these initial constraints accordingly for the next steps.

Viewing and understanding Street Art in Wellington established that there were a number of different visual styles present. These varied artists and works were inspired by a number of different topics and themes. These works were scattered throughout the city and gave inspiration to the final outputs outlined in the next chapter.

Taking AR tracking to various locations in Wellington proved that the majority of the surfaces tracked well in AR. This is due to the fact that the urban environment is quite rough, allowing for features to be recognised and digital planes to be generated quicker. This allows for a number of different concepts to be considered all over the city. 
These findings allowed for the designs of the next chapter to be developed with a better understanding of the technical and contextual ways Digital Street Art can make artistic use of the street in Wellington. This allowed for the Digital Street Art designed to better engage with the public space and provoke responses due to a better understanding of the space used, and the range of contexts that could be addressed. 


$$
\begin{array}{r}
\text { Chapter } 4 \\
\text { Digital Street Art }
\end{array}
$$


This chapter consists of fully developed speculative Digital Street Art, informed by both the contextual research in Chapter 2 and the experimentation in Chapter 3. These works were developed with the aim of adding to the findings of the previous chapter, leading to conclusions around the practice of creating Digital Street Art using AR.

The literature review discussed how the use of public space allowed Street Artists to engage with the public, making provocative statements and eliciting responses using the street as an artistic resource (Riggle, 2010). It also covered how mobile Augmented Reality allows digital content to be placed within a physical space, allowing it to be placed within the context of the street. Digital Street Art can therefore make artistic use of the street to engage the public with digital content through the use of Augmented Reality.

The free experiments in the previous chapter showed how the possibilities of the technology could be used within the context of Wellington. These experiments allowed for a better functional understanding of how the designs could be placed in the street and make use of the street as an artistic resource.
Two core Wellington issues were chosen as the focus for the designs in this chapter:

- The Wellington Rental Crisis

- $\quad$ Plastic Waste and its effect on marine life

These led to the development of several designed responses, informed by the previous chapters. These were designed to confront viewers with social and environmental issues at specific locations around the city, hopefully provoking response. These locations informed the designs, remaining internal to their meaning and engaged the public space. This allowed the Digital Street Art designed to remain Street Art (Riggle, 2010), whilst still using the technology of Augmented Reality (AR). 


\section{Cats and Mice}

The concept for this scenario was developed from personal experience as a student living in Wellington for the past 5 years. Wellington has long suffered from issues in its housing market, with rental properties specifically proving incredibly volatile. This is particularly apparent in low income and student housing. (Cann, 2017)

The competitiveness of Wellington's rental market has resulted in an increase in price, making even the poorest quality flats above the budget of many students and low-income families.

There are frequent reports of poorly maintained properties with unhealthy living situations, not just in Wellington but across New Zealand. News sources reporting on mould covered flats with no insulation and poor maintenance which are being leased to low income renters and students around the country (Mcneilly, 2018) (Burry, 2016). Driven by an increase in demand, and a decrease in listings, the market is only compounding the issue. This has led to higher prices and more competition for even the most dilapidated flats (Matt, 2016) (Cann, 2018a).

Exaggerating the issue further appears to be a large number of the landlords renting properties in this market. With multiple reports indicating a desire to extract as much money as possible from renters, regardless of quality and living situations. This can be seen in price

gauging auctions on rental properties, seeking to drive renters against each other, bidding over the asking price (Matt, 2016). Another instance of this appeared at the beginning of 2018, when the government allowance for students went up, and reports of rental increases in the same amount were reported across the city (F. C. and G. Campbell, 2018).

There were a number of ways in which a response to this issue could have been designed as a work of Digital Street Art. Key to designing this issue would be the tone and delivery of the work. Would it be designed for students, lawmakers or the landlords themselves? No matter the target audience, it would still be designed to provoke thought and consideration towards the issue. 
Some initial concepts discussed the idea of recreating the poor conditions of flats in public locations, with the opportunity to specifically target more upmarket locations, government buildings or university grounds. This would draw attention to the poor standard we tolerate in rental properties, a standard not tolerated in other spaces.

For this concept was to succeed it was important to make the design visually appealing enough to view and interact with while communicating its message. For this reason, I chose to use a mushroom that I could imbue with a sense of fantasy, rather than just straight mould and damp conditions.

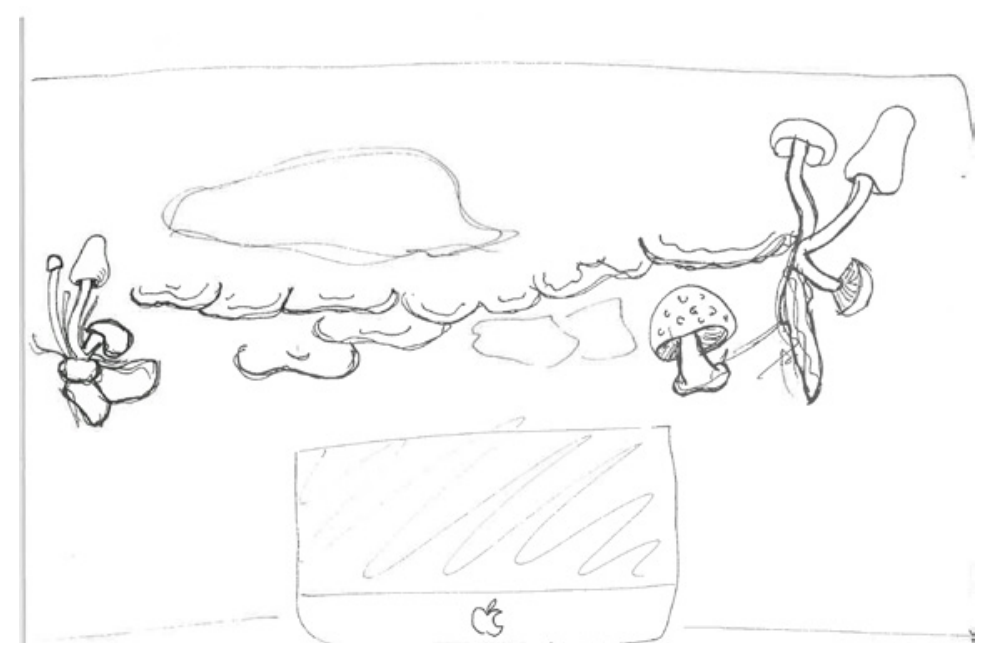

Figure 48: Concept of mushrooms above a desk on campus

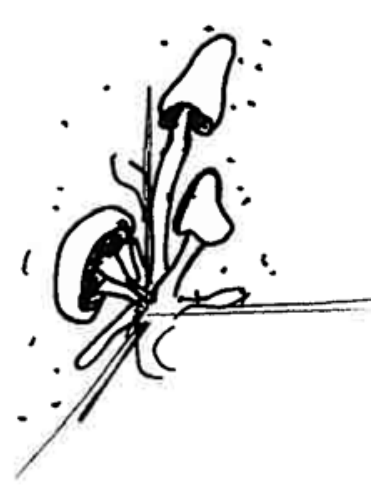

Figure 49: Mushrooms beginning to grow in the corner of a room

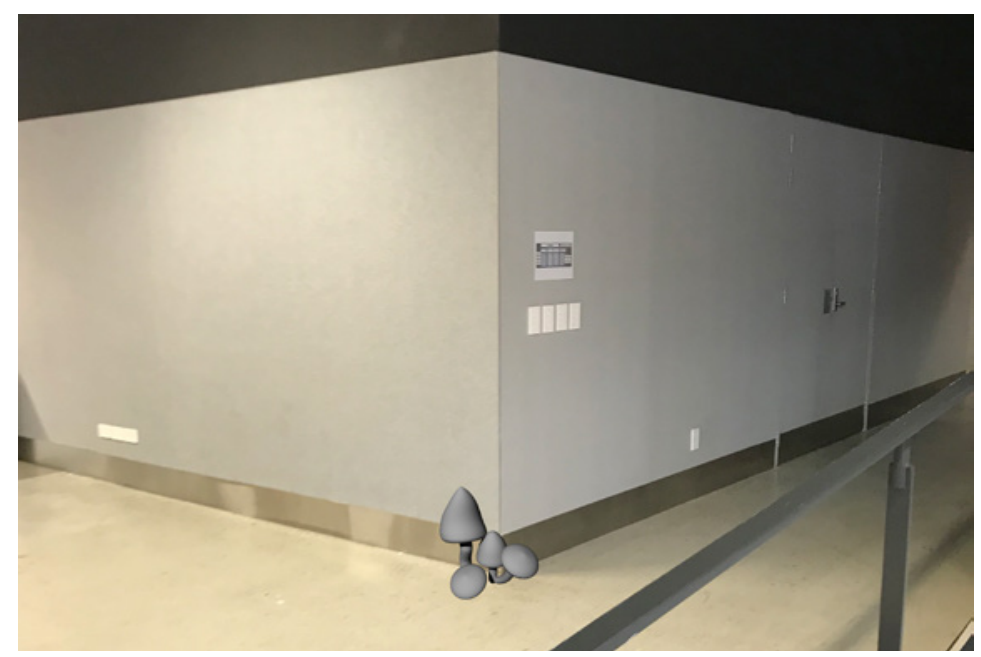

Figure 50: 3D sketch models used to represent the above sketch 
Another opportunity for concept development was to target landlords and confront them for the issues facing these residents. In doing this there was the opportunity to use caricature to represent and confront these landlords. This concept allowed for some creative expression from the perspective of a student that has experienced these issues. Landlords are effectively the gatekeepers of these student or low-cost suburbs, pushing their properties and getting people into them, without any care for the properties themselves. A high turnover is expected in student properties because many leave at the end of the year based off the quality of the flat. This results in landlord constantly having to find tenants to push their properties on. This concept would have been a 3D character acting as a gatekeeper to a notorious Wellington suburb known for its poor living conditions and volatile rental market.

The possible locations for this were varied, with Lower Cuba, Mt. Cook, Kelburn, Aro Valley and Mt. Victoria all being considered. However, in testing this concept and developing it, it was tested in Aro Valley park.

This park is at the entry to the suburb, and many students walk through it, to and from the university campus.

If this concept was to continue the first step was to test a layout for the AR design, making sure that the landlord could stand within a physical space and the tracking would still work. This was a simple matter given the relatively small ground surface area of the content, however it was still important for choosing a flat enough area within the park (shown in Figure 51).

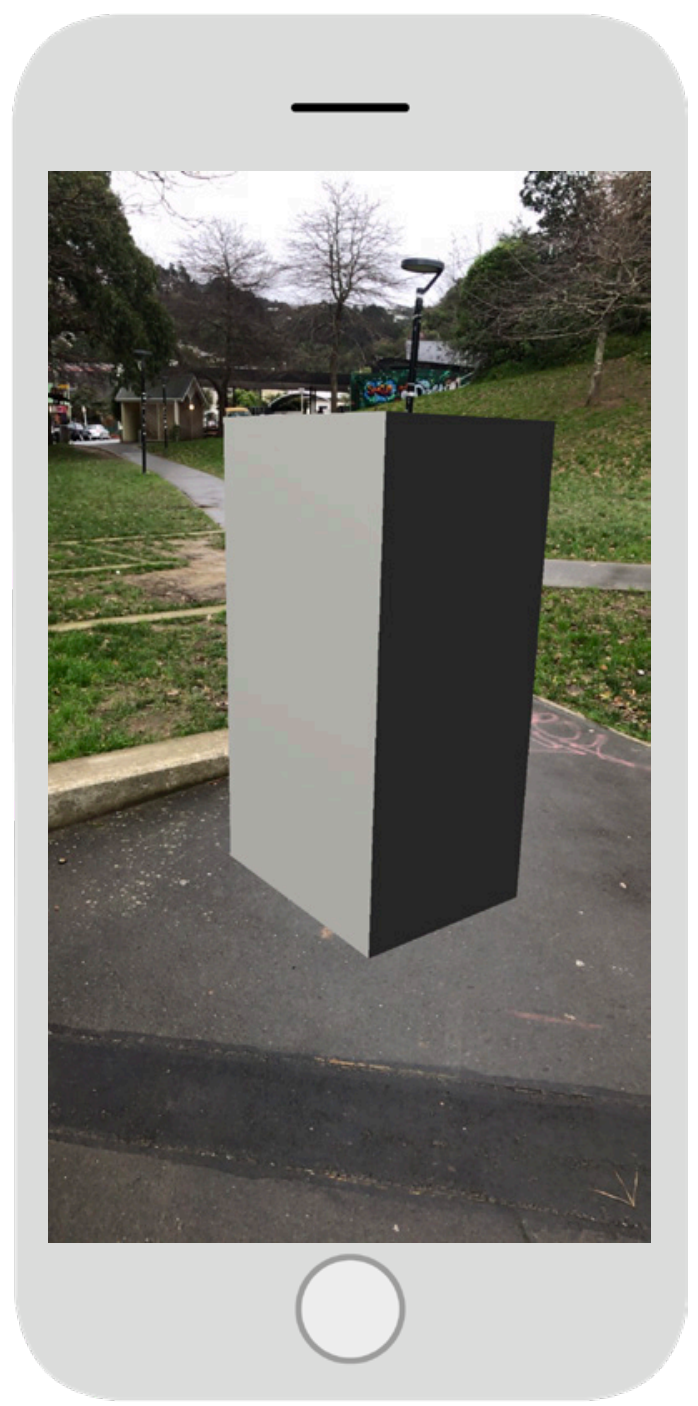

Figure 51: Greyboxing for a buman scale digital model 
To represent a landlord, I wanted to use caricature and exaggerated features, playing off the stereotypes of a greedy character, profit focused and overtly wealthy. This would allow for a design that used non-specific physical characteristics that are fully interpreted based on personality.

The "Fat Cat" character was chosen to develop, since it felt like a stronger metaphor. This character was then developed and modelled in Maya.

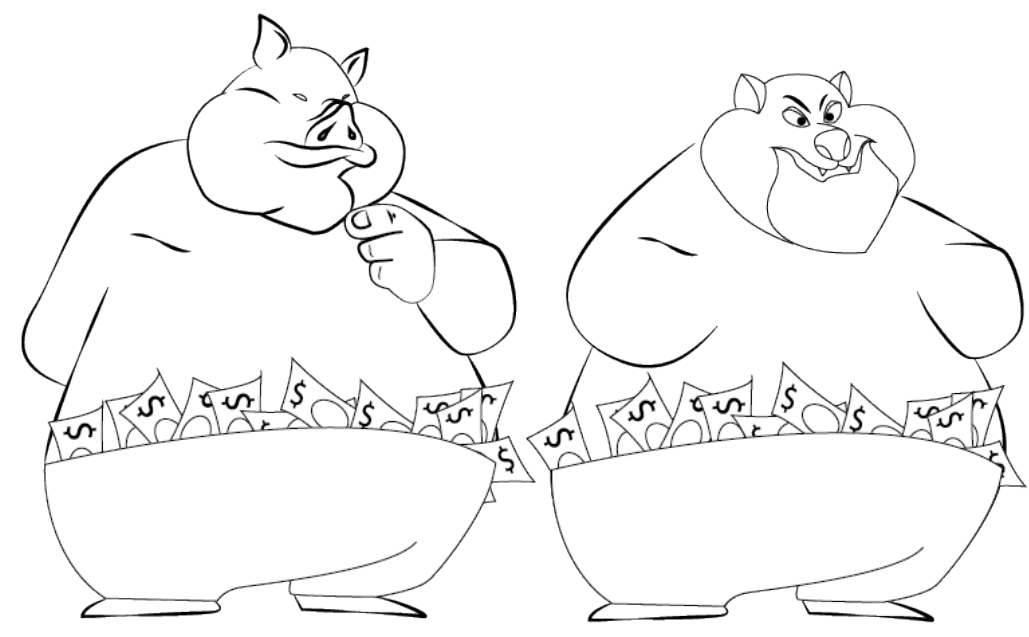

Figure 52: Considerations for charicature
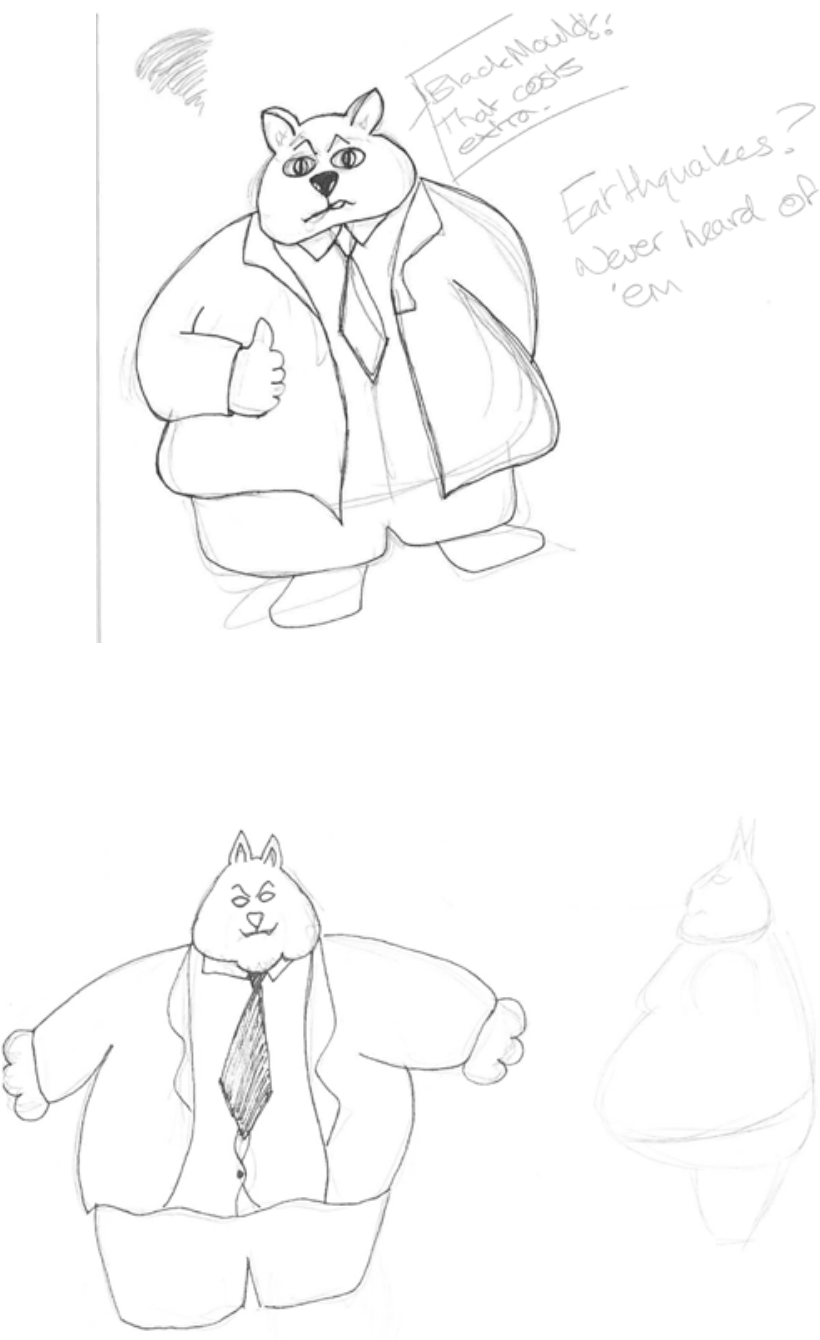

Figure 53: Sketches of the Cat Design 


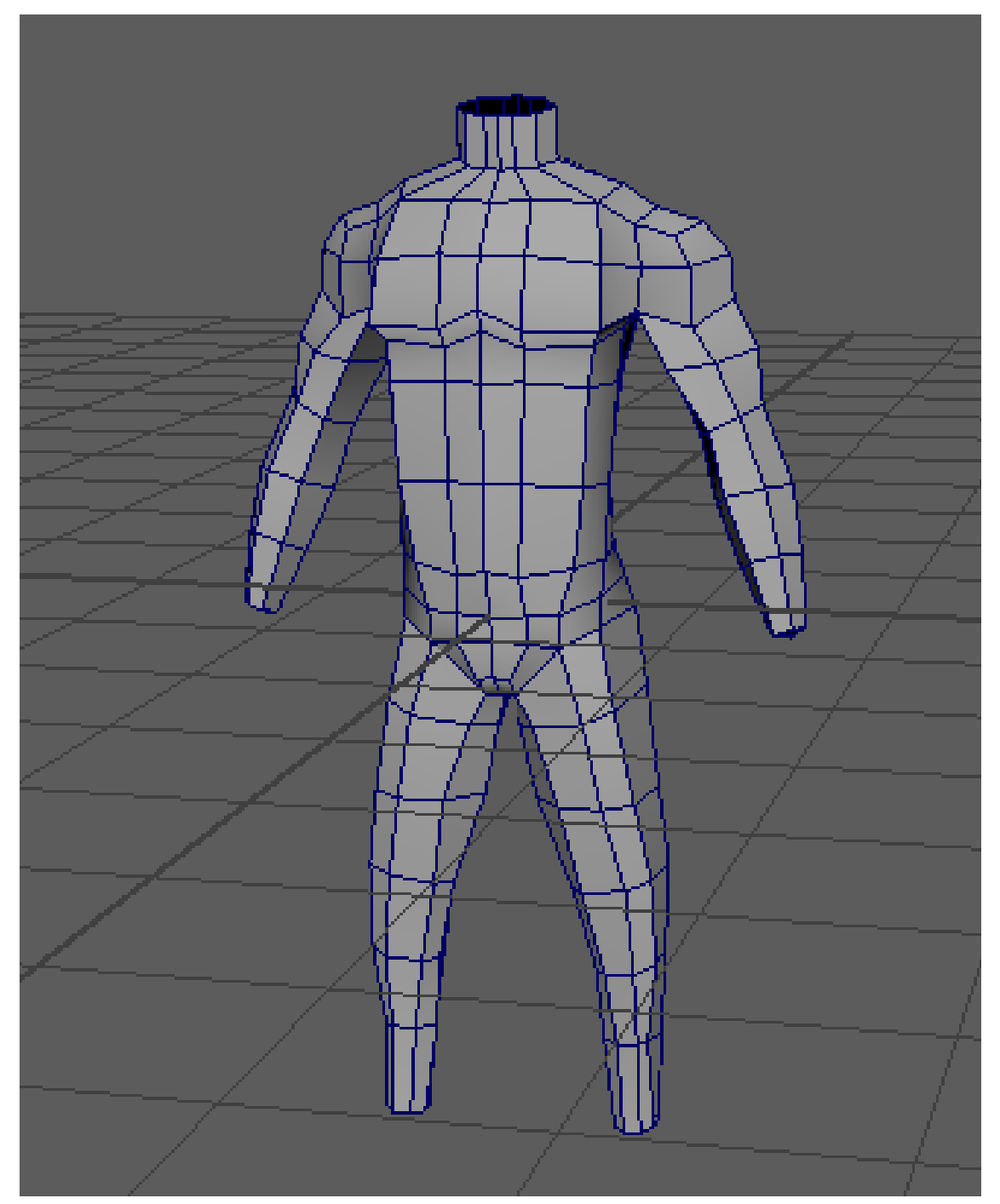

Figure 54: Generic humanoid model that was made as a base, this meant that it could be used later if another bumanoid figure was required

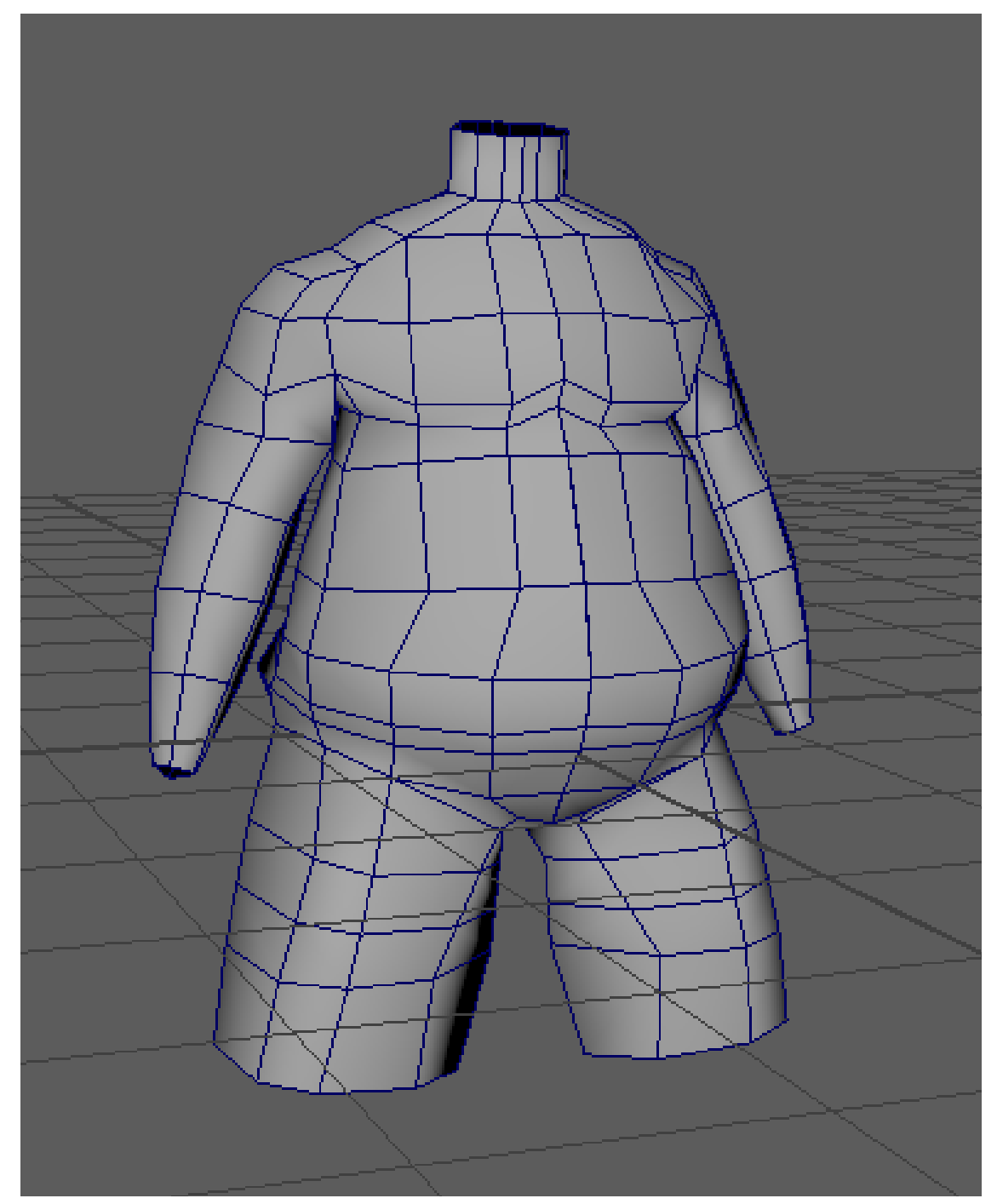

Figure 55: Editing the model to take a larger shape 


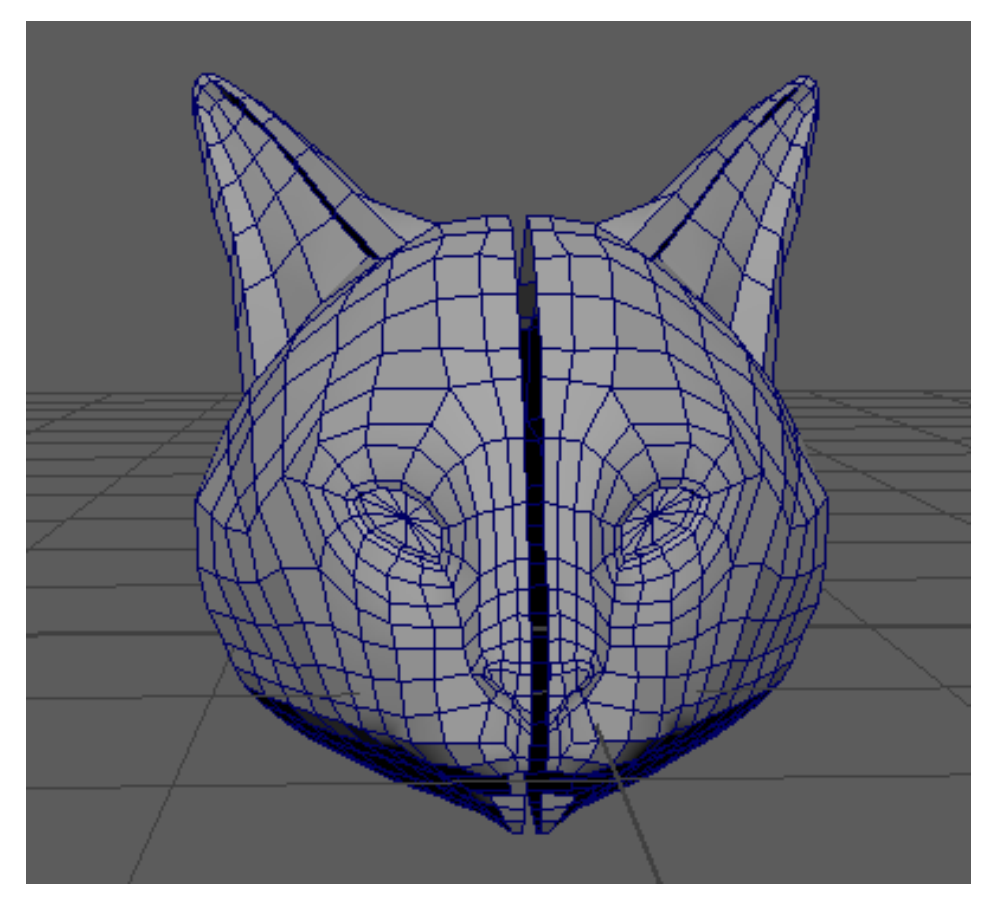

Figure 56: Initial modelling to get the structure of a cat's face

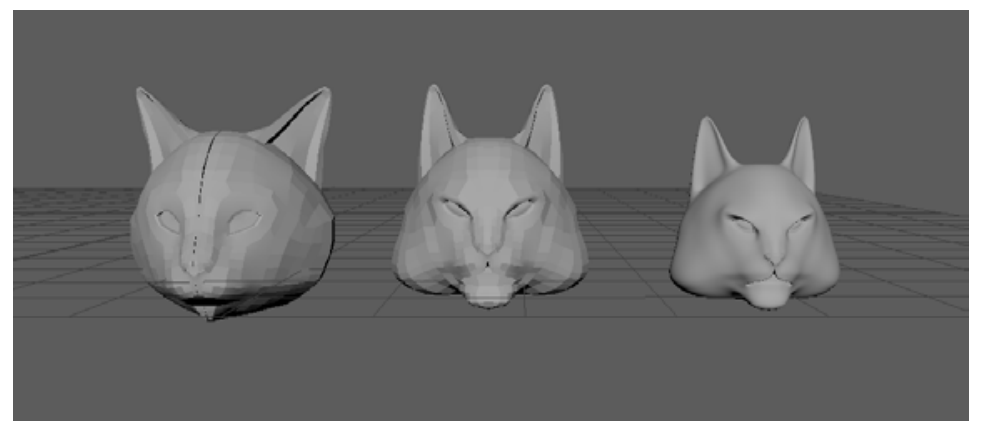

Figure 57: Editing the cats face to make a more caricatured style

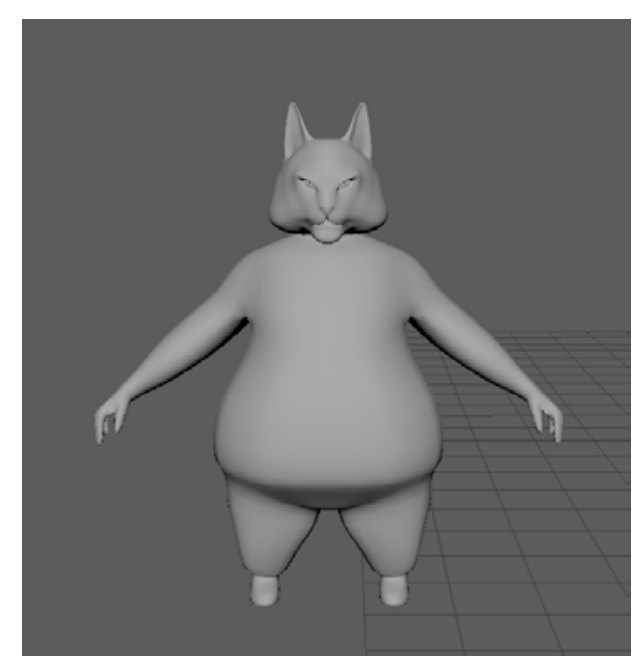

Figure 58: Full model of the fat cat

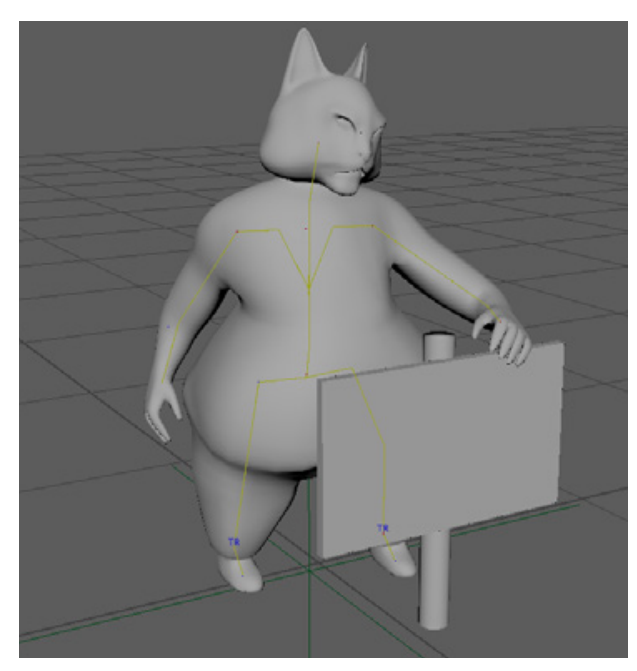

Figure 59: Fat Cat model, rigged, posed and with a prop 


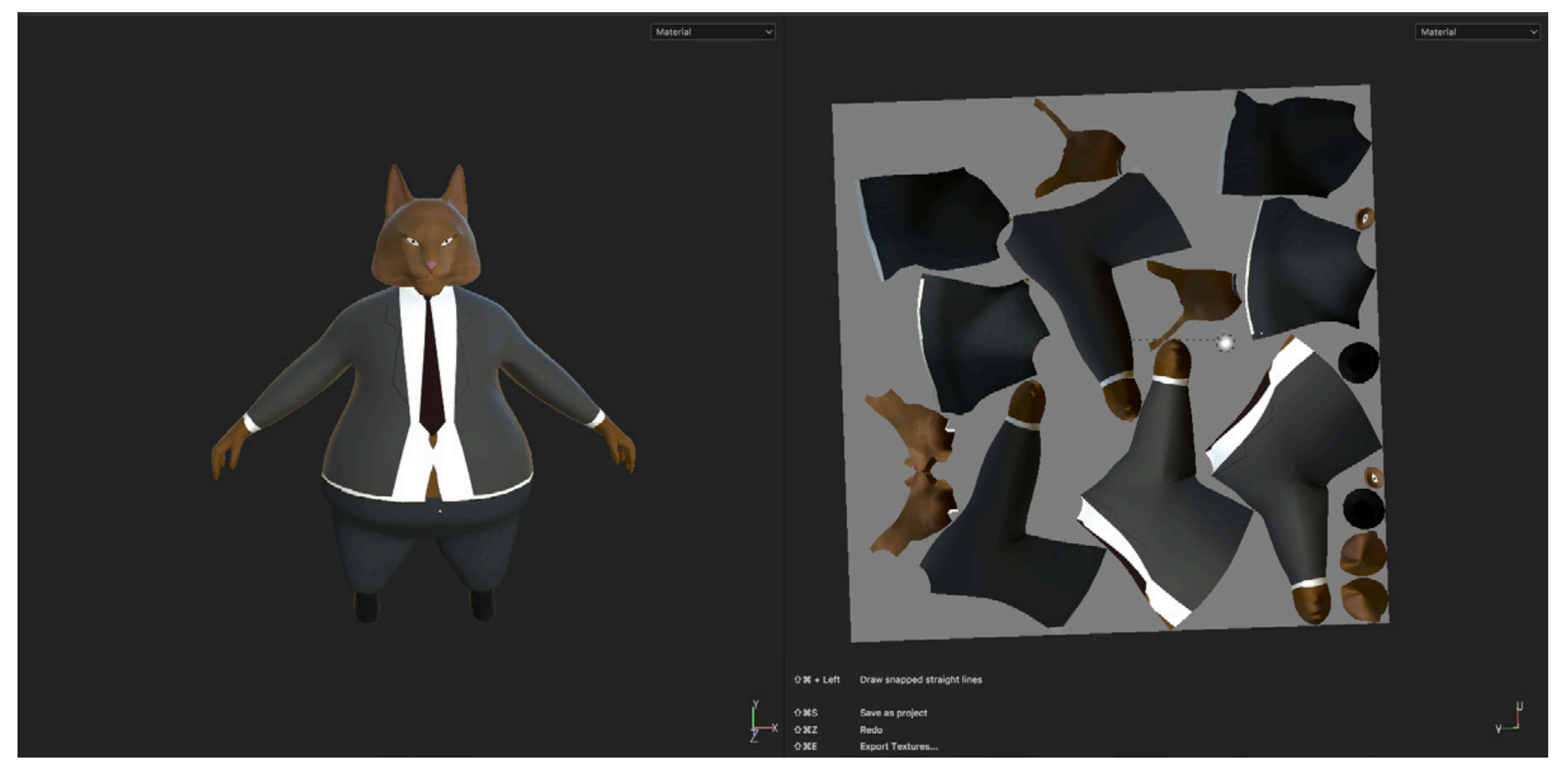

Figure 60: Texturing the cat character 
A rental sign was added to the digital model, adding to the character's identity. A pile of poorly maintained signs was then added behind

character model to indicate that they had rented several properties and didn't care about them once they are rented.
This design concept was developed enough to be placed in AR, however upon further reflection it did not draw strong context from the location it was in. This context was loose, and did not use its specific location as an artistic resource (Riggle, 2010). For this reason, I decided to explore the scenario in other ways.

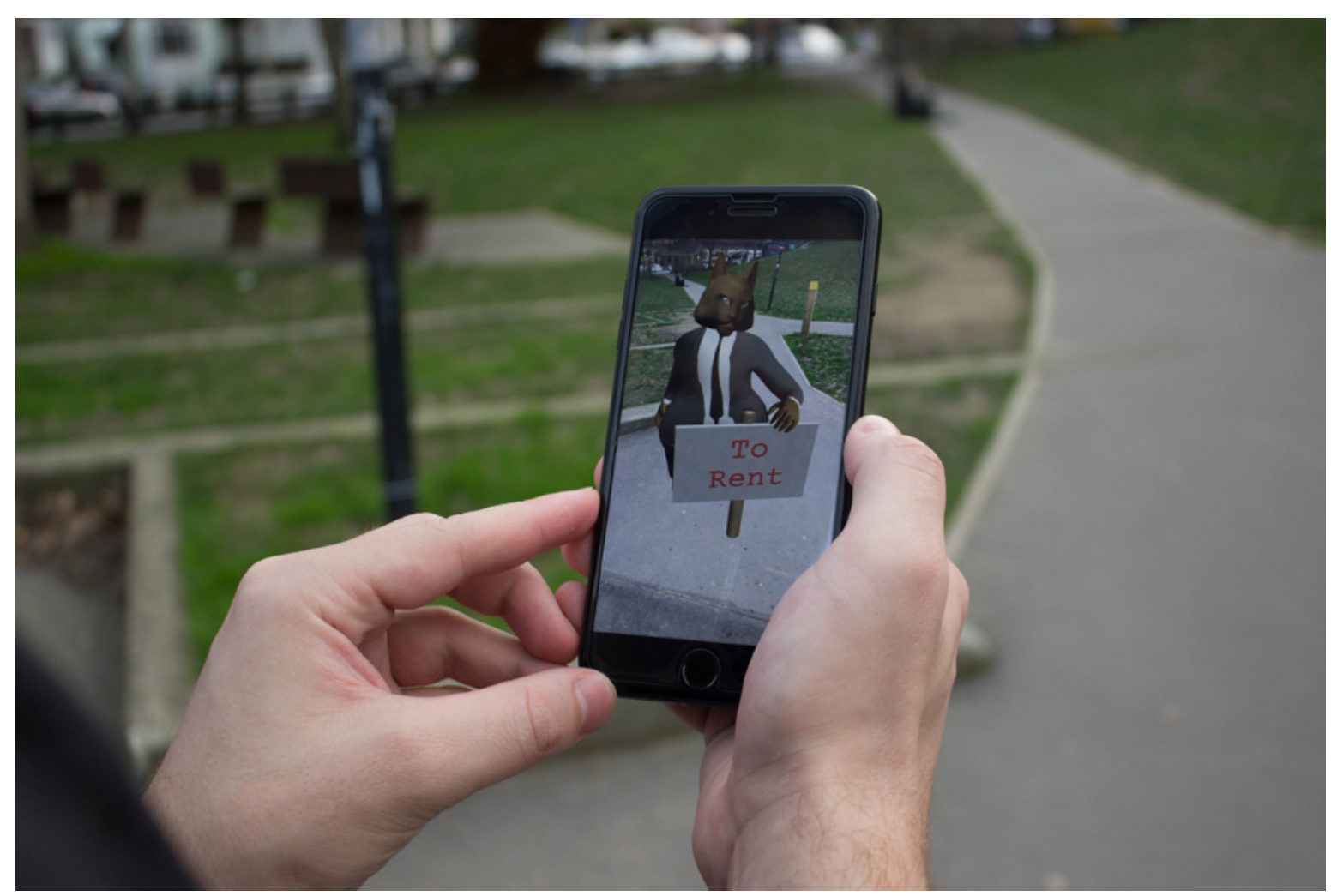

Figure 61: Showing the Fat Cat in AR through the device 


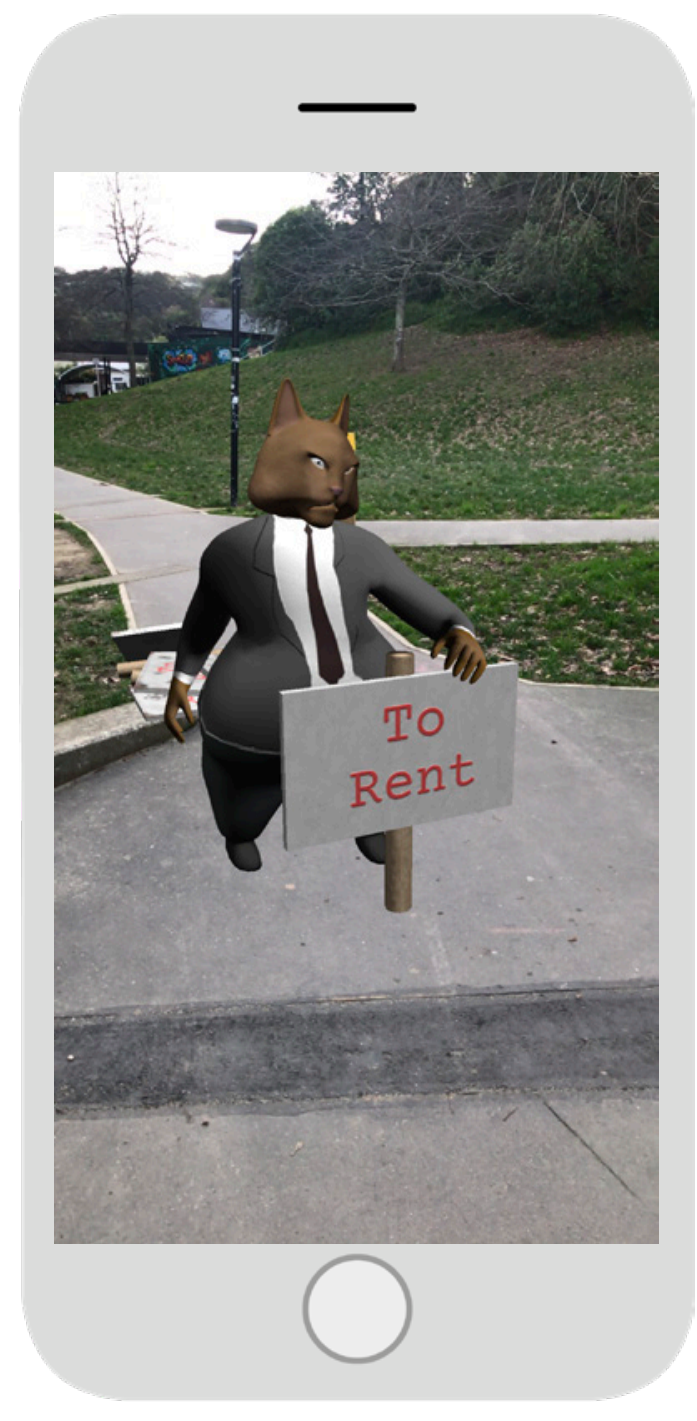

Figure 62: Fat Cat in Aro Park

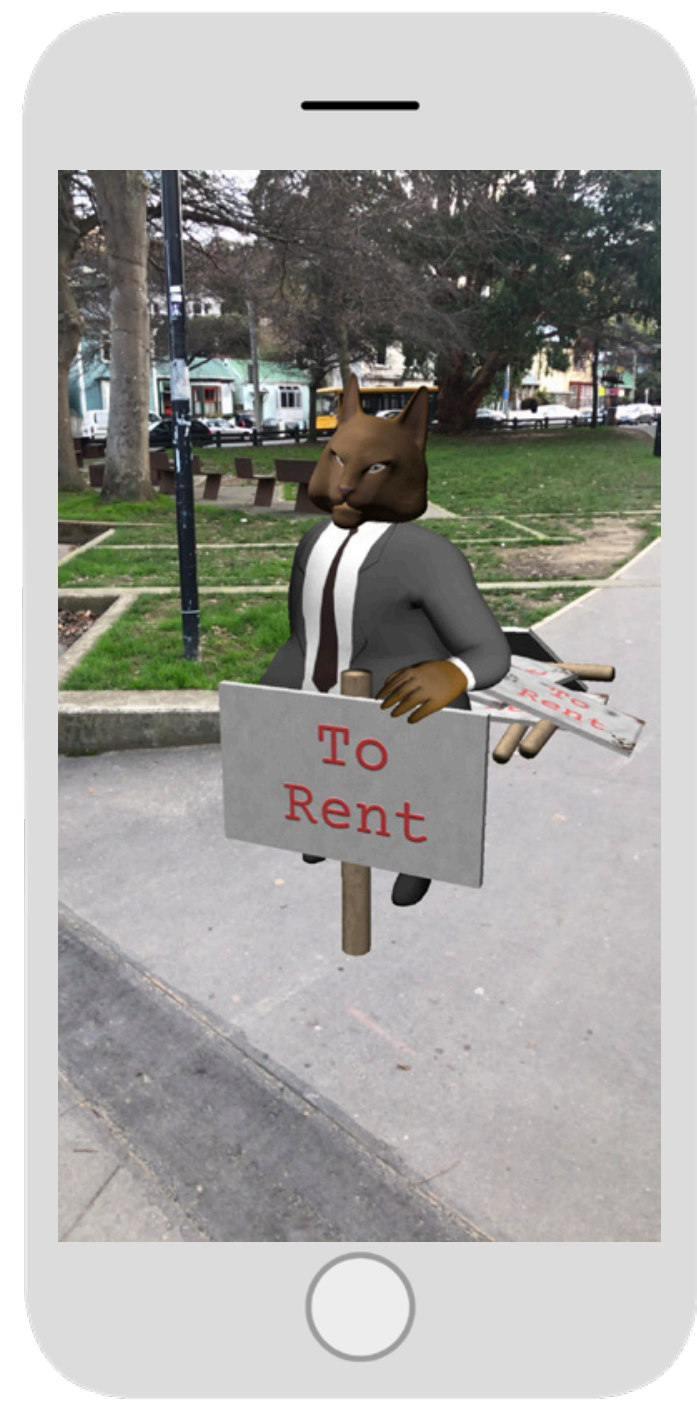

Figure 63: Different angle of the cat in Aro Park

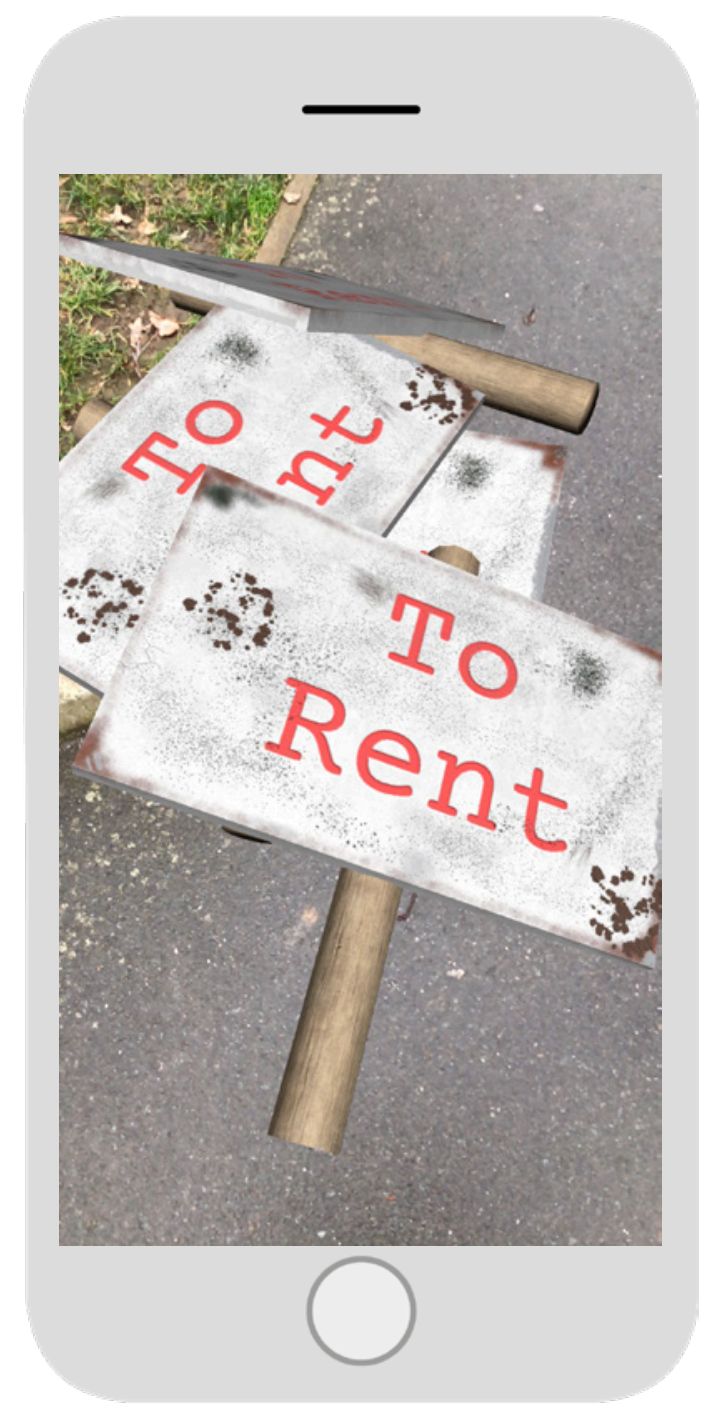

Figure 64: Details bebind the character 
One of the benefits of AR is its ability to reclaim the visual space without damage to property. This could mean that could reference private property without causing damage and issues with legality. AR's nature as a digital medium, means that it can be updated regularly and reference online information.

It was also decided that I could reference and discuss the tenants as the focus. They are the ones that have to suffer and deal with the issues in this rental market. This also resulted in a shift in thinking, addressing more specific ways content in AR could be used to address locations, but also in ways that could address multiple locations with the same meaning.

For this reason, I chose to develop this concept further, and it split into two different works. These were both focused on the rental markets in Wellington, however they were more aimed at the audience of students and renters affected by the issue. This allowed the designs to have some practical use to the viewers, potentially using provoked response to improve the lives of those that view the work

The first concept was to draw attention to the prices involved with trying to flat in Wellington. Addressing the disparity between incomes within the city, and the rent collected by landlords. In particular many students rely on the government allowances or living costs provided. While this amount was raised recently, it is still not enough for many to both pay rent and live comfortably. I decided to explore this using simple text responses to rental listings and prices. These would replace the listed cost with the "true" cost of the rent.
MORE 'THAN WHAT YOU EARN

\% OF MLIDIAN INCOMN a SIUDEN'I ALMWANCWS

\section{TOO MUCH}

Figure 65: Text explorations of the "true" cost of a rental property

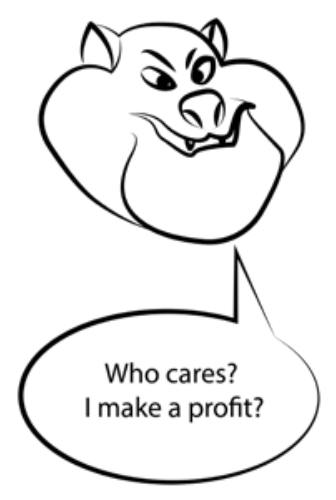

Figure 66: Sketch of a Fat Cat landlord stamp that could be placed on properties 
I decided to apply these $2 \mathrm{D}$ explorations to the $3 \mathrm{D}$ sign generated earlier, and place it in AR. This work would serve as both an artistic commentary on income disparity and could even warn potential tenants at locations about what the rental price that is listed actually means for them long term. In this way, this piece of Digital Street Art draws from the location it is in and supplements that location, adding to the visual space of the street. To create an example of this work in action, I found a Trademe listing and used its location and price as the base for converting to the "true" cost, based on the Studylink student allowance amount: \$230.12 (MSD, 2018). A more generic sign was also generated, that could be placed outside any expensive rental listing without the need to do any calculations.
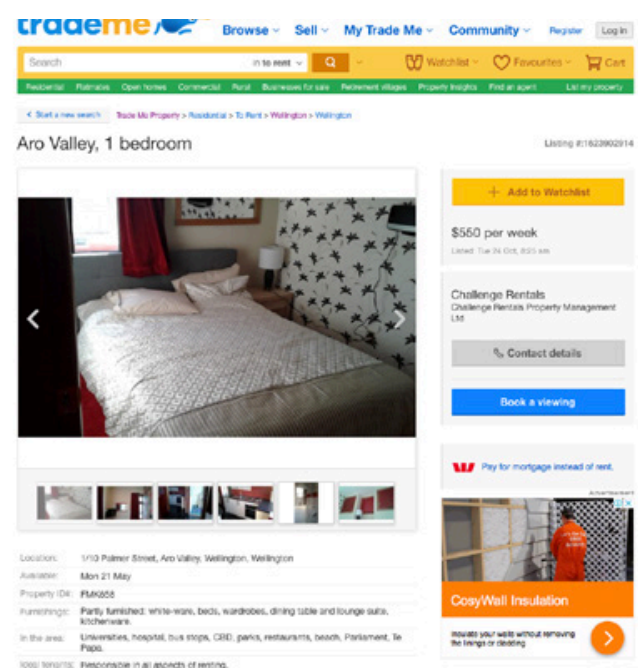

Figure 67: Screenshot of Trademe Listing

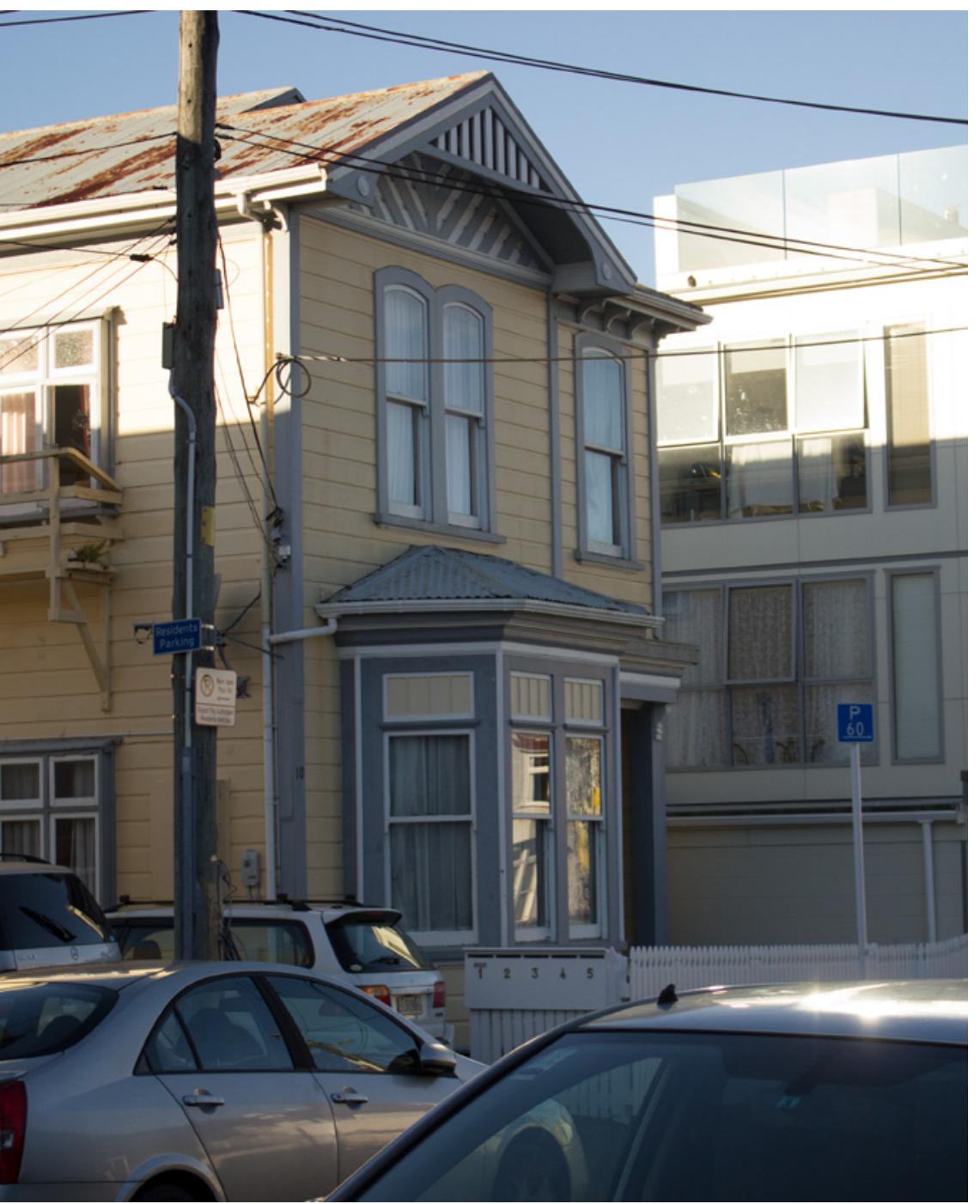

Figure 68: Photo of listed property 


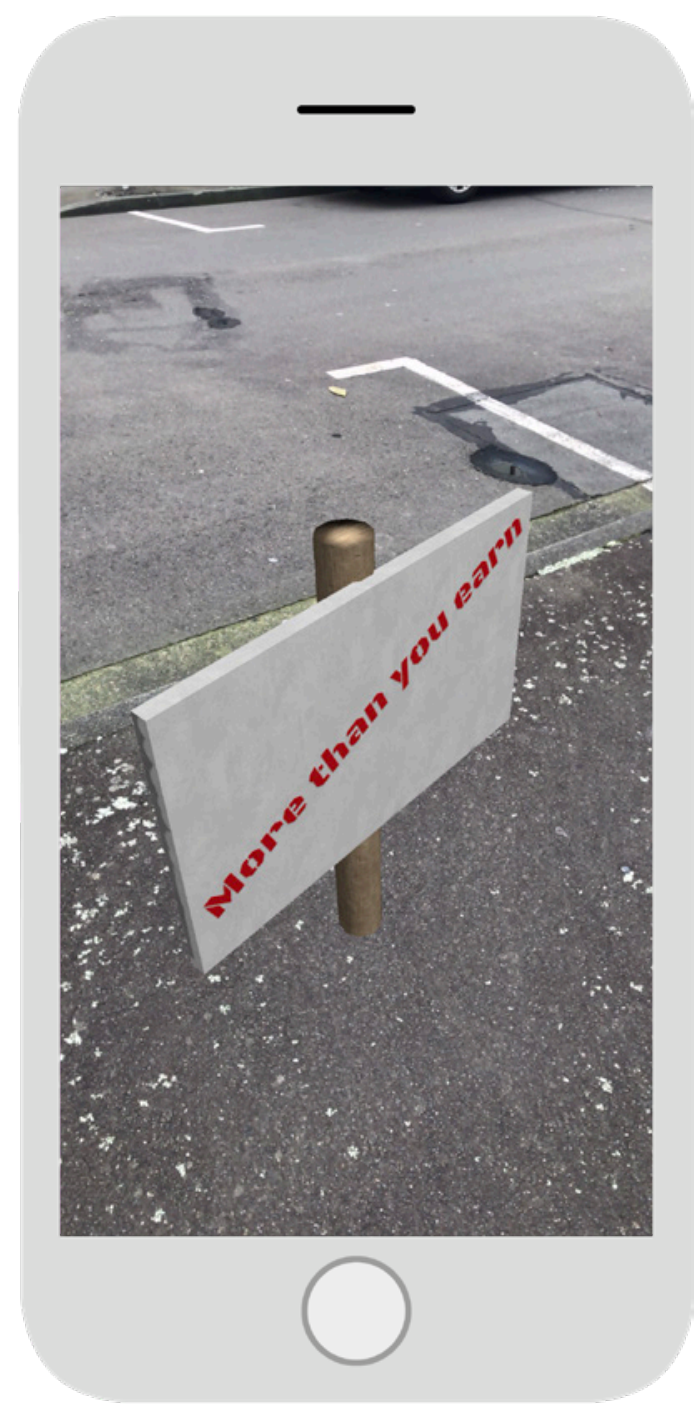

Figure 69: Generic design that could be placed anywhere in front of any property with a high price

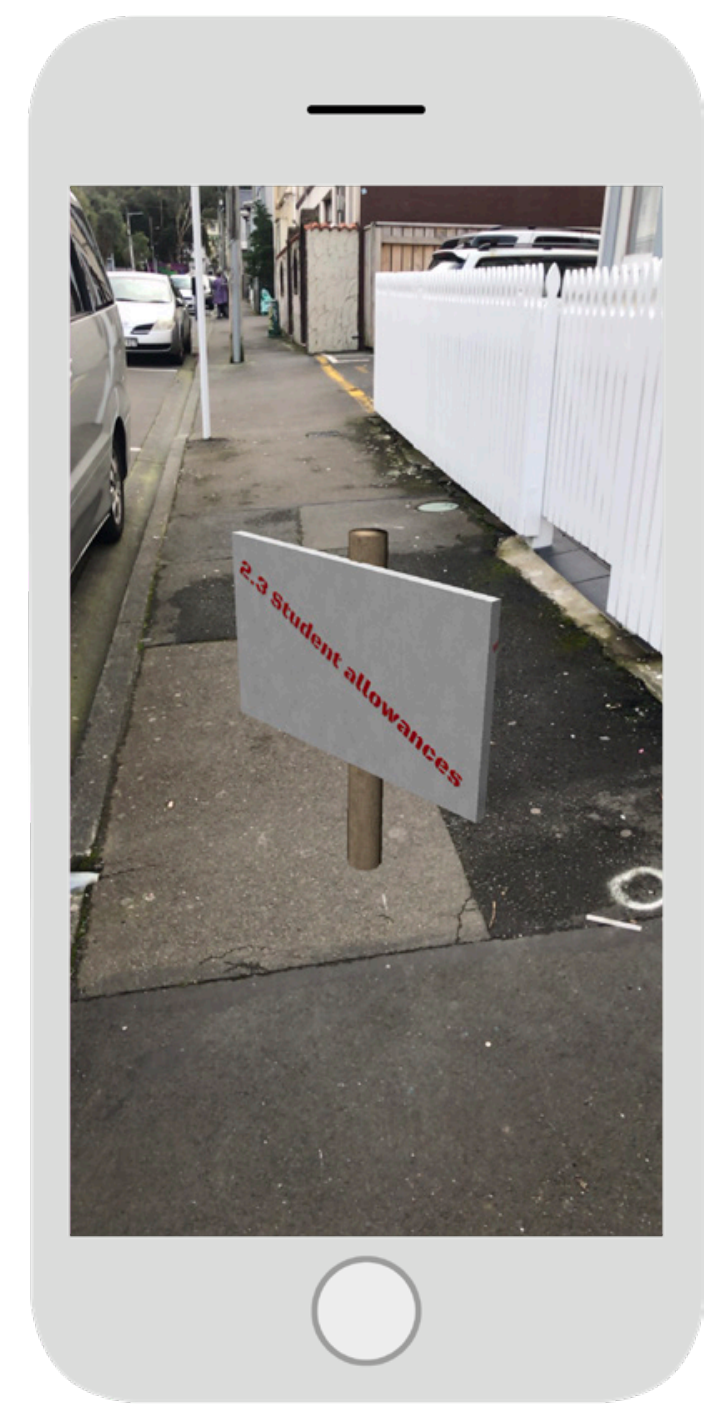

Figure 70: Rental Sign placed on the street in front of listed property

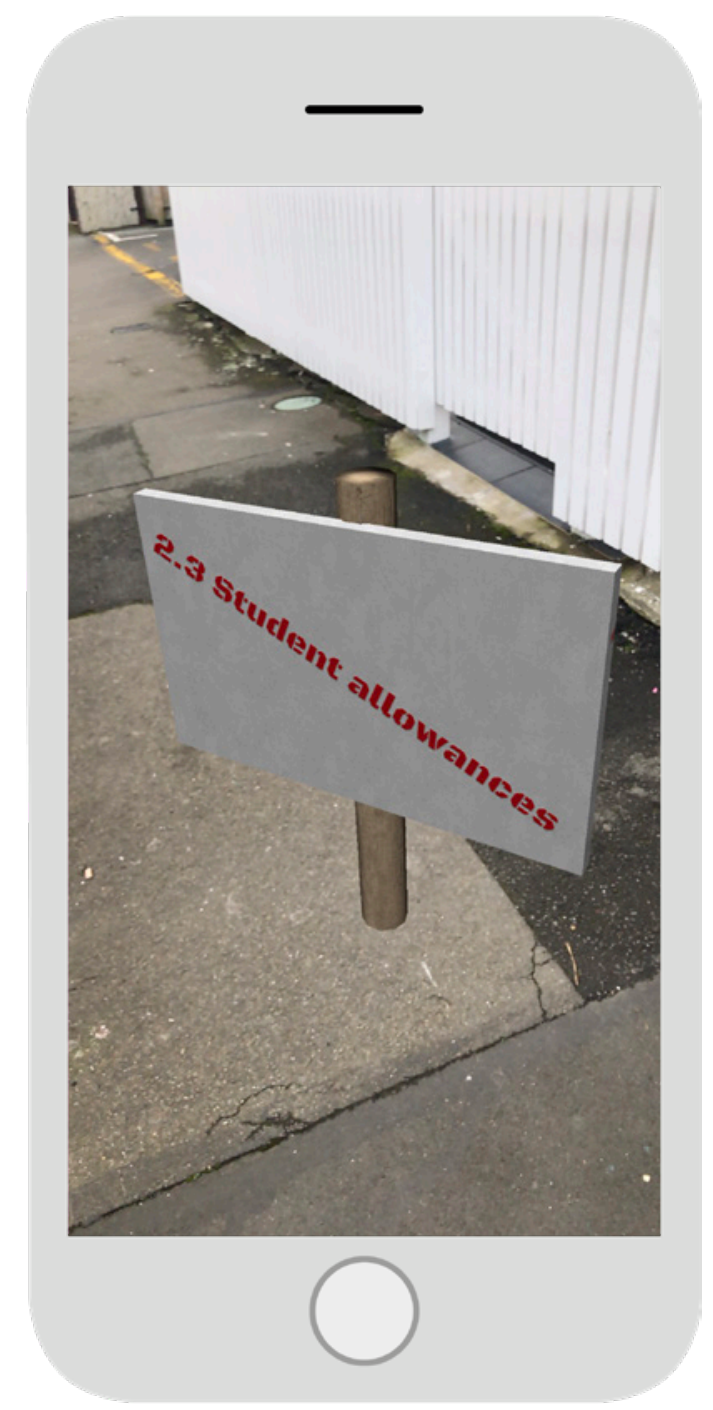

Figure 71: Price of the trademe listing converted to student allowances 
Using the concepts already created as a base, the next exploration was focused on the maintenance issues in the Wellington rental properties. Drawing on the concept of the fat cat landlords, I represented tenants as mice living under their thumb. These concepts were also generated in two dimensions, this allowed for a more traditional Street Art style to come through, which strengthened the metaphor and referenced inspiring artists.

The final design of this concept resulted in a series of "badges". These could be placed in front of properties with specific poor living conditions, either by viewers, or based on rental listings and reports to the artist. A badge was designed for common and prevalent wellington issues; however, this list could be expanded in the future. The issues these badges addressed were:

1. A landlord that was unresponsive to issues and maintained the property poorly

2. Properties that are damp

3. Properties that are cold and have no insulation or heating

4. Properties that are earthquake prone

The final designs for these badges are shown in Figure 72.

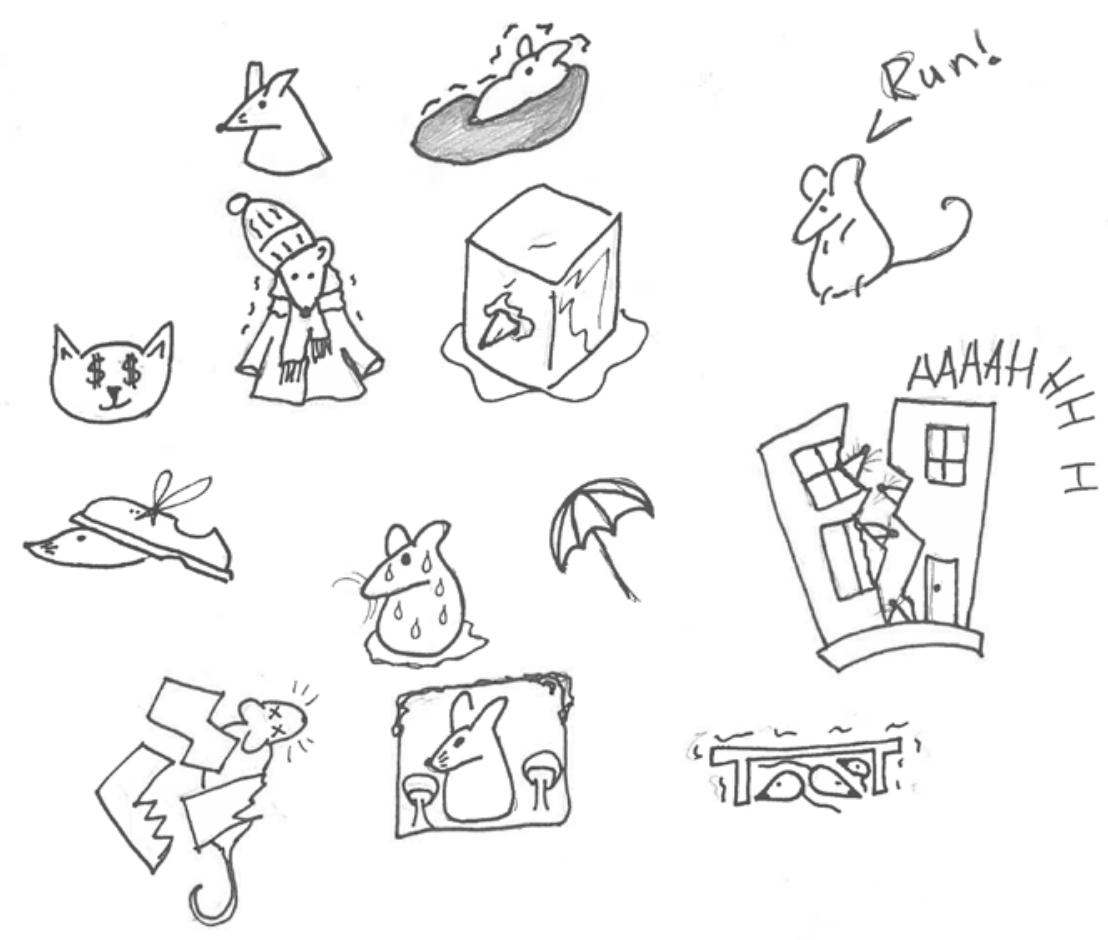

Figure 72: Concept sketches for mice badges 


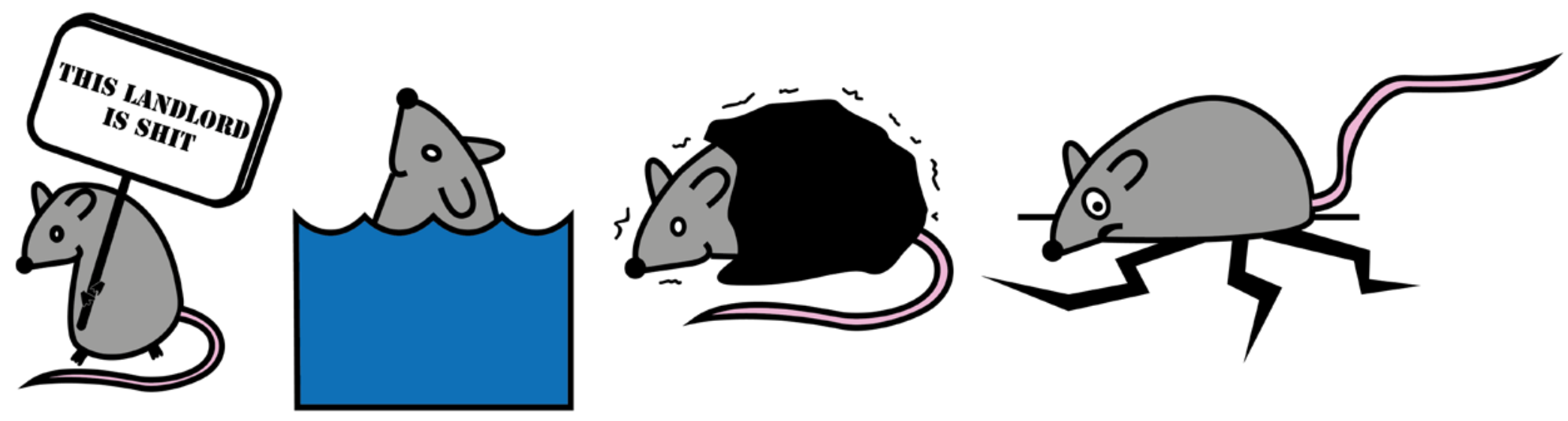

Figure 73: Flat quality badges

65 


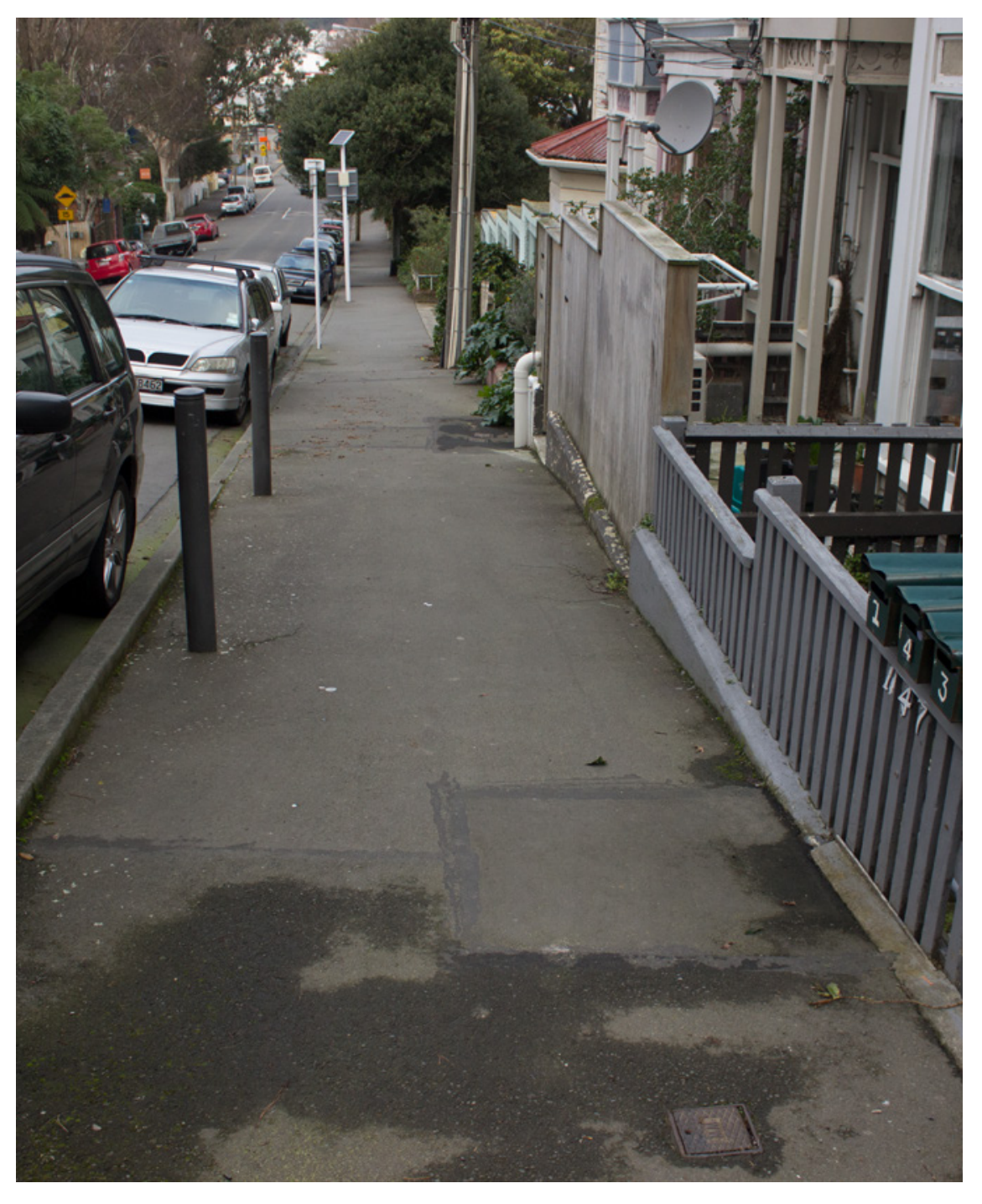

Figure 74: Street Outside the property

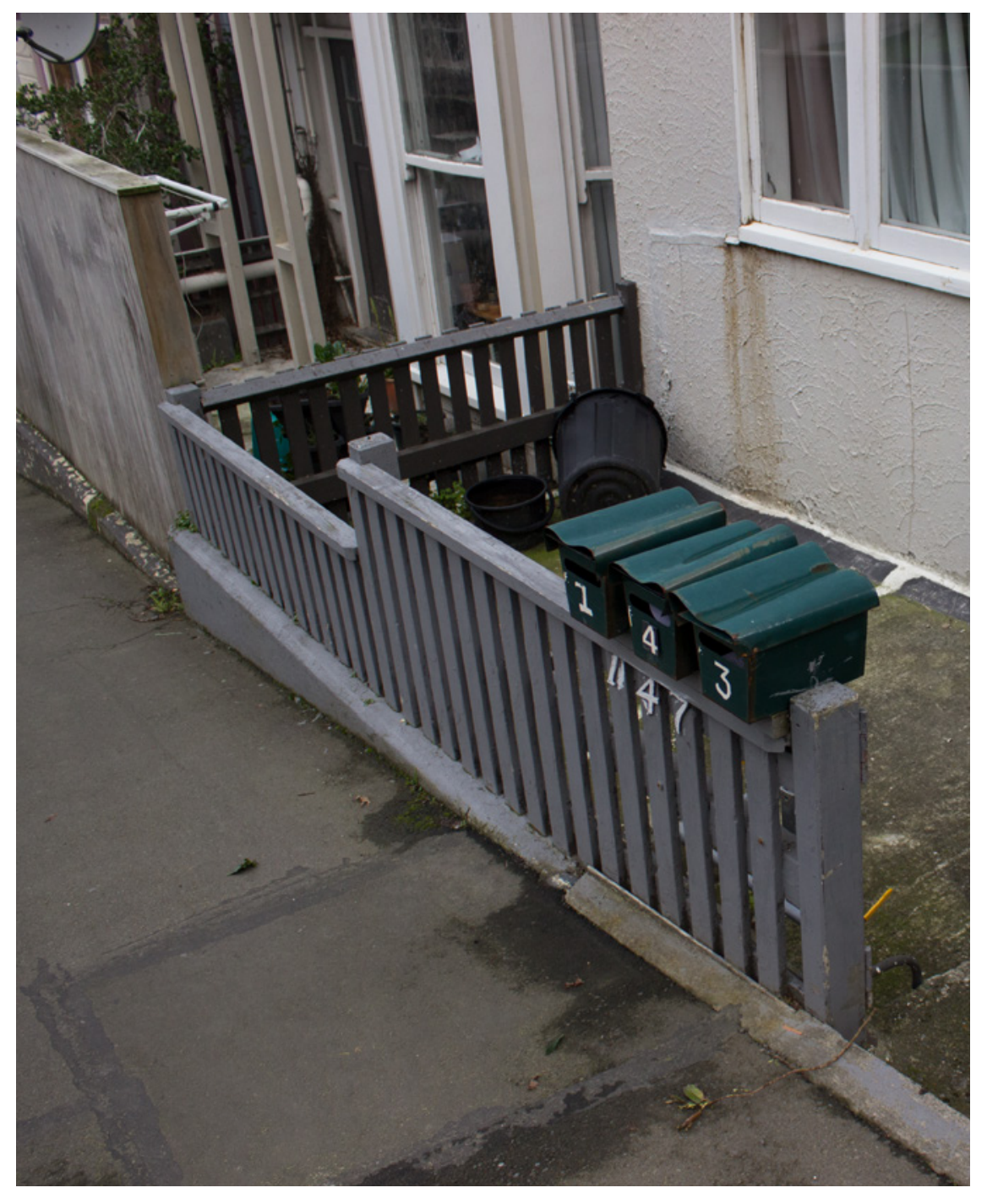

Figure 75: Area in front of the property that could be used as the site for content 


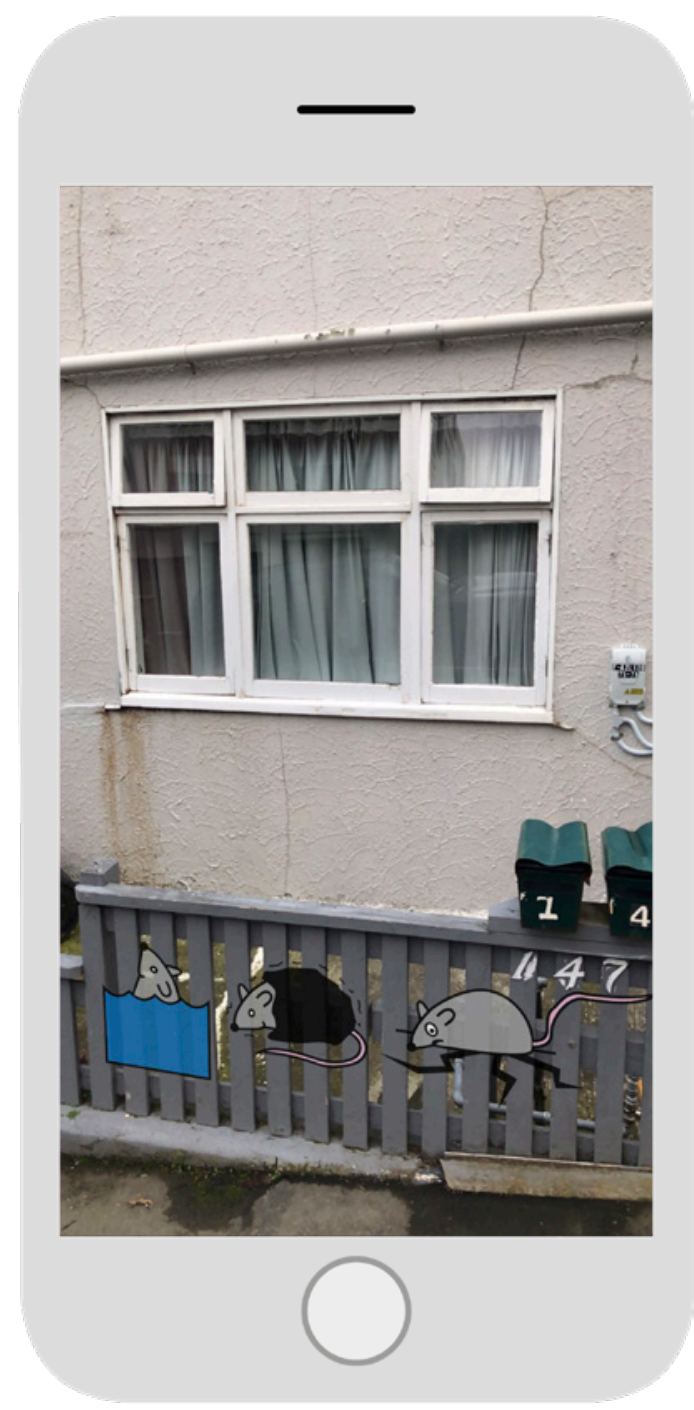

Figure 76: Badges labelling the property with its quality issues

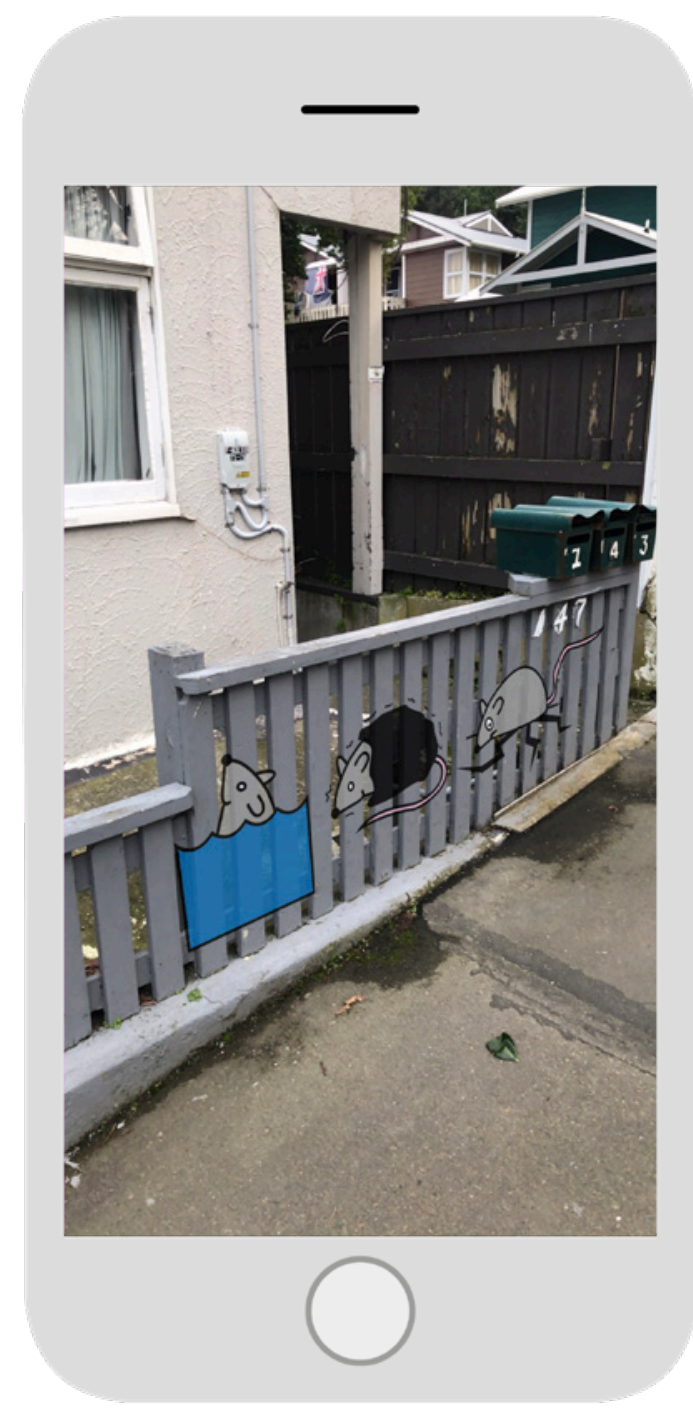

Figure 77: Badges could be added to the front fence

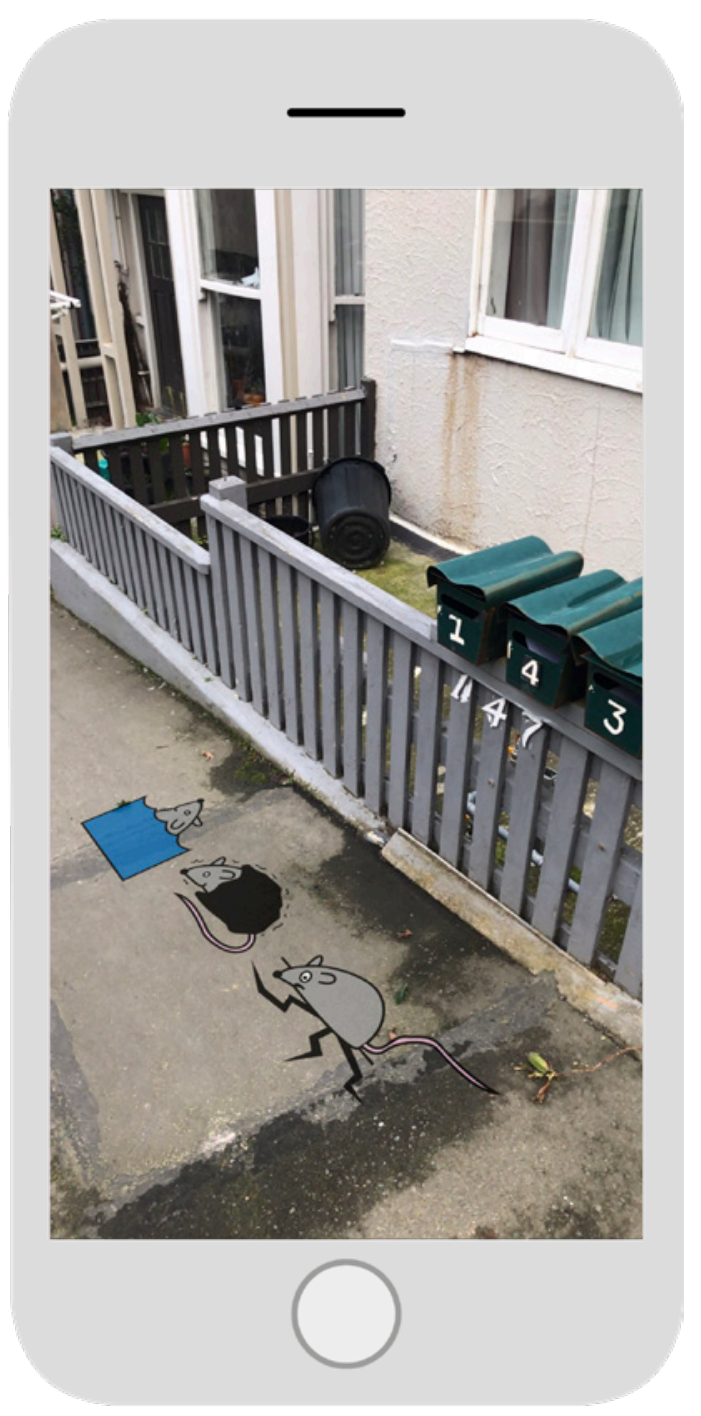

Figure 78: The badges could also be placed along the ground 


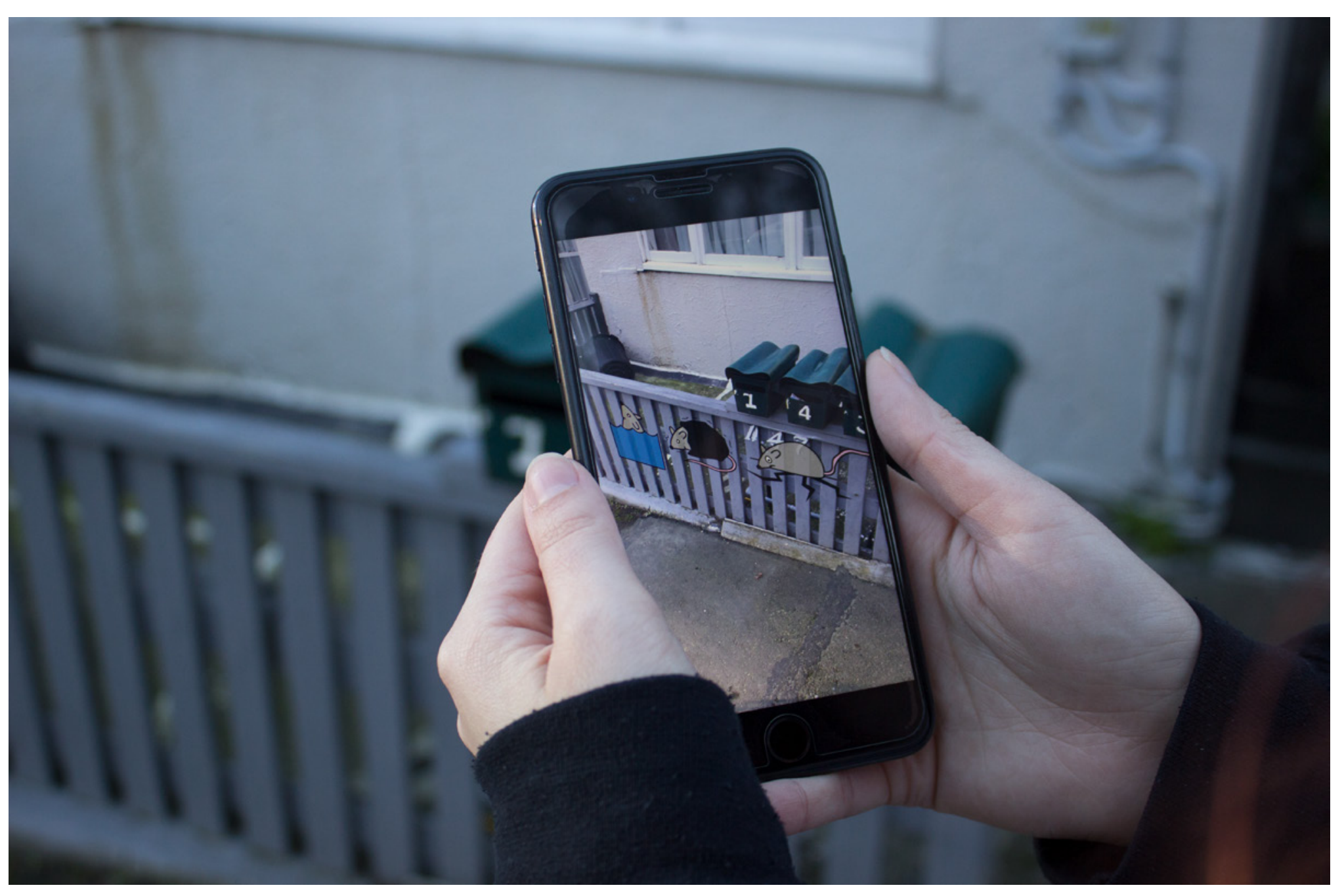

Figure 79: View of the Mice badges through the device 


\section{Reflection:}

This design scenario afforded many different concepts and potential topics for exploration. Visualising these issues aimed to spark debate and discussion amongst any that viewed these designs. These works drew on their physical location, and using AR, became part of the visual dialogue between the viewer and the location. However, the concepts developed from specific information about the location were made stronger by their specificity and allowed for more interesting reflection. These made more use of their location on the street to draw meaning and context, using the street as an artistic resource informing their design (Riggle, 2010). These works would serve as accusations, warnings, and expressions of frustration to those that view to them. Provoking different responses from a range of viewers in the public space

\section{Future Considerations and possible development}

While these works drew from their locations and served as examples of Digital Street Art. This scenario could be developed further in a number of ways: More animations could have made the characters more alive and could have been used to create more narrative driven designs. More interaction could have allowed for some interesting ideas relating to how it actually feels to interact with a landlord or rental property in this market.

Tying these designs to live data or user responses was beyond the technical scope of this research, however there are several possibilities within this space. For example, tying the rental signs to data on rental listings online directly, or allowing users to submit data online that could be linked to the mice designs. 


\section{Disposable Consequences}

While addressing current issues, it would have been remiss not to address one of the biggest issues in today's world; climate change and human waste. This issue is a broad one and would require a more focused approach to refine into a developed concept. This work could be viewed by many members of the public and therefore could be used to raise awareness and inspire change. I decided to focus on issues that an individual could respond to, potentially changing habits or actions that may affect the environment. For this reason, I decided to narrow my focus to plastic waste.

Wellington is a coastal city, and its waterfront area is very popular with both residents and tourists. Recognising this, this scenario will address plastic waste in the ocean, specifically marine debris. Marine debris, or litter, refers to small plastic waste found in the ocean. This remains one of the most pervasive and solvable pollution problems affecting oceans (Sheavly \& Register, 2007). Marine debris can impact wildlife, habitat and even human health (Thompson, Moore, Saal, \& Swan, 2009). Increasing awareness and knowledge about the issue is a fundamental method for improving ocean quality and preventing pollution (Sheavly \& Register, 2007).

Concepts for a scenario responding to this issue began with considering various sea creatures and how they may be affected by marine debris.

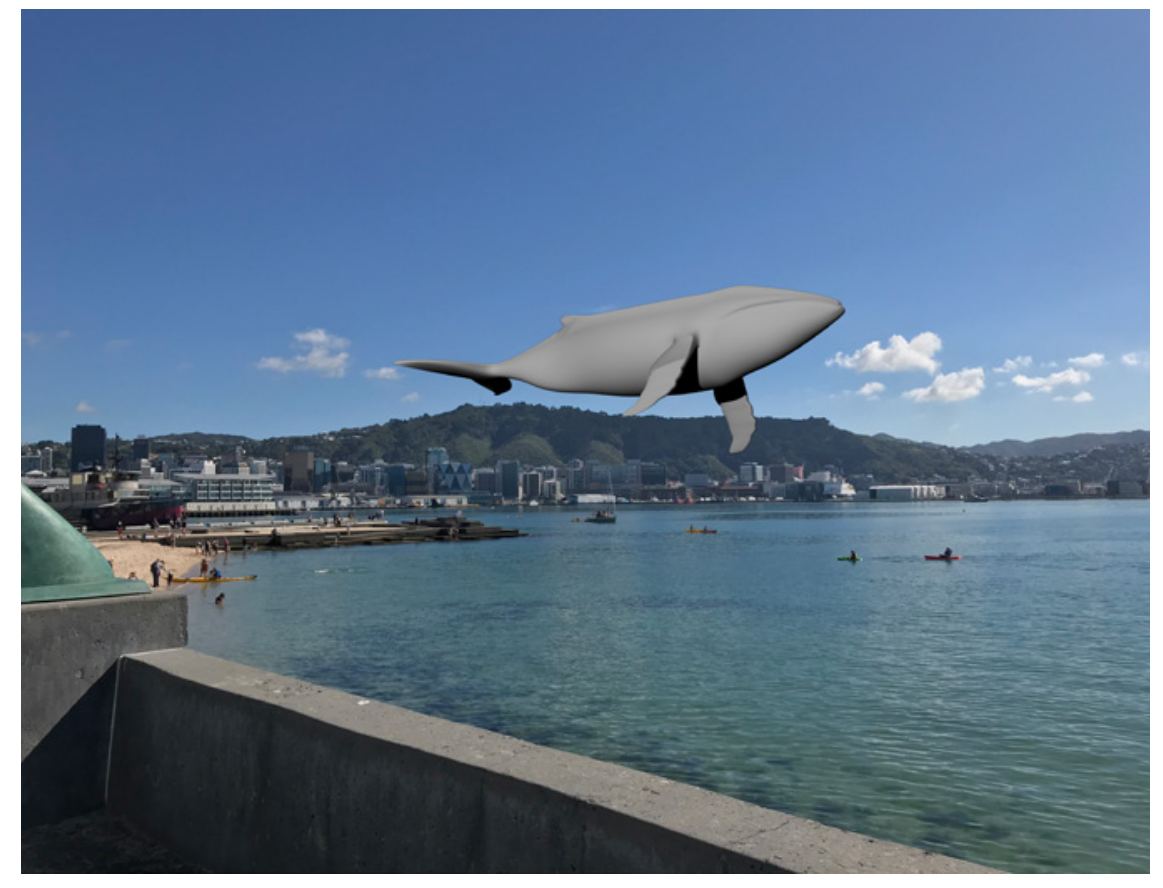

Figure 80: A very early mockup of how marine life could be placed into AR 


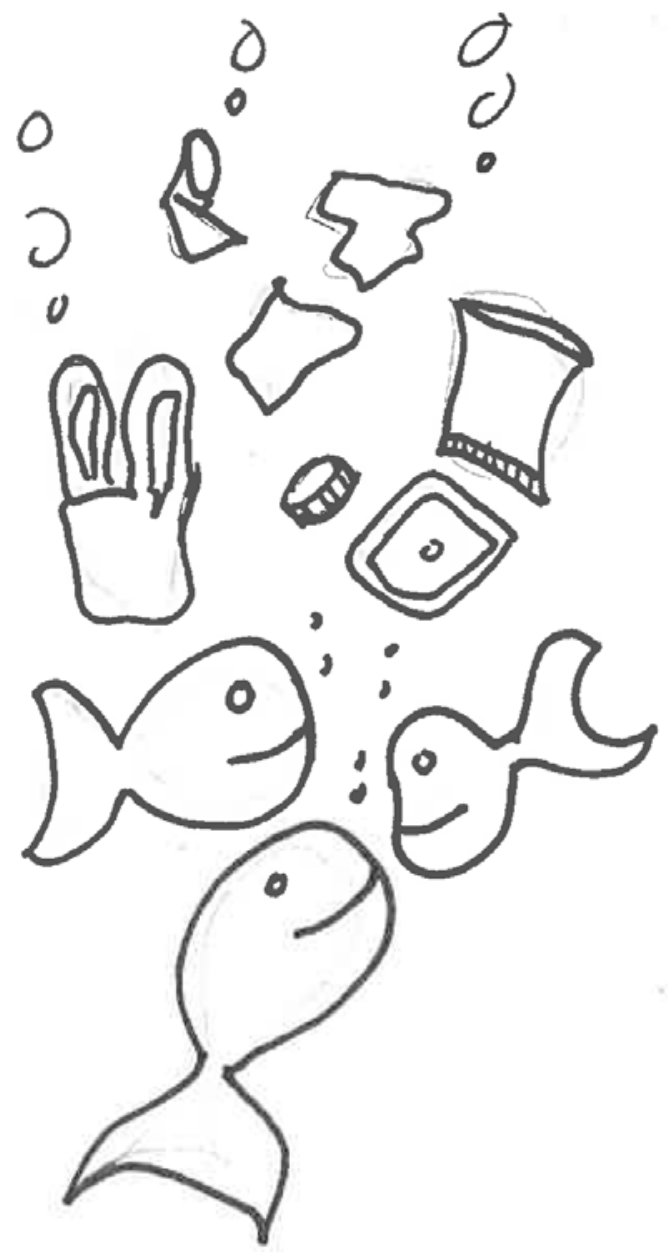

Figure 81: Early sketch of plastic and marine life

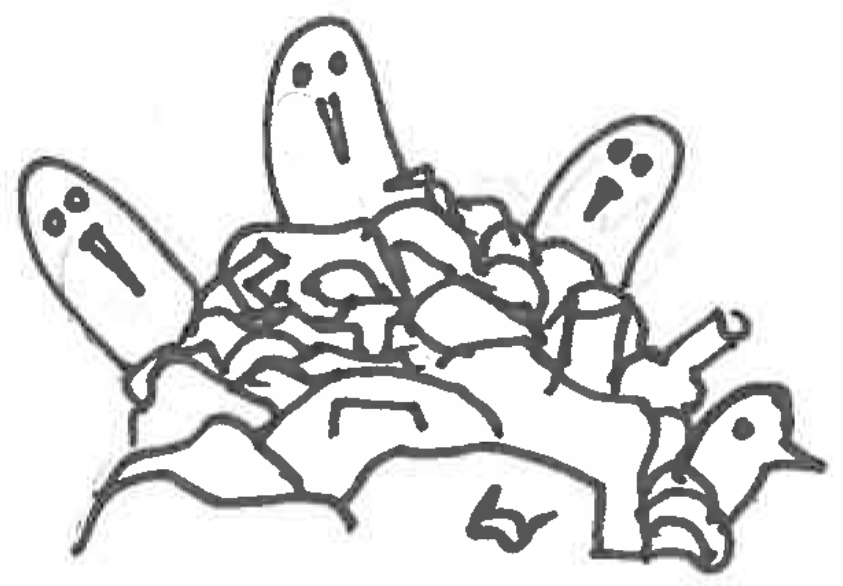

Figure 82: Early Sketch showing marine birds and waste

To narrow the focus of this scenario, I researched some causes of marine debris and plastic pollution. In this it was discovered that single use plastic was a major source of plastic pollution in New Zealand (Sustainable Coastlines, n.d.) 
Wellington waterfronts became plastic straw free in April 2018 (Cann, 2018b; Wellington City Council, 2018). However, there are still many places within the city that sell and use them. Sustainable Coastlines collects 10,000 straws from Wellington beaches each year (Wellington City Council, 2018), proving that the issue is still a serious one.

A Wellington City Council initiative showed that the majority of restaurants were open to a ban and that over 10,000 straws were collected from Wellington beaches alone. Plastic straws get lodged in turtle breathing cavities and can cause serious harm (Plastic Pollution Coalition, 2015). Using this as an inspiration for the design, I decided to make a turtle "smoking" a straw, comparing the consequences of our actions to a bad habit, creating the metaphor for humans forcing an unhealthy bad habit onto other creatures.

After some initial sketches, a 3D exploration of the turtle began, this could allow the turtle to float and swim around viewers on the waterfront.

From this I decided to experiment with adding more waste that could float around the turtle. For this I used a mixture of my own assets and free assets found on the Unity Assets store through CC licensing.
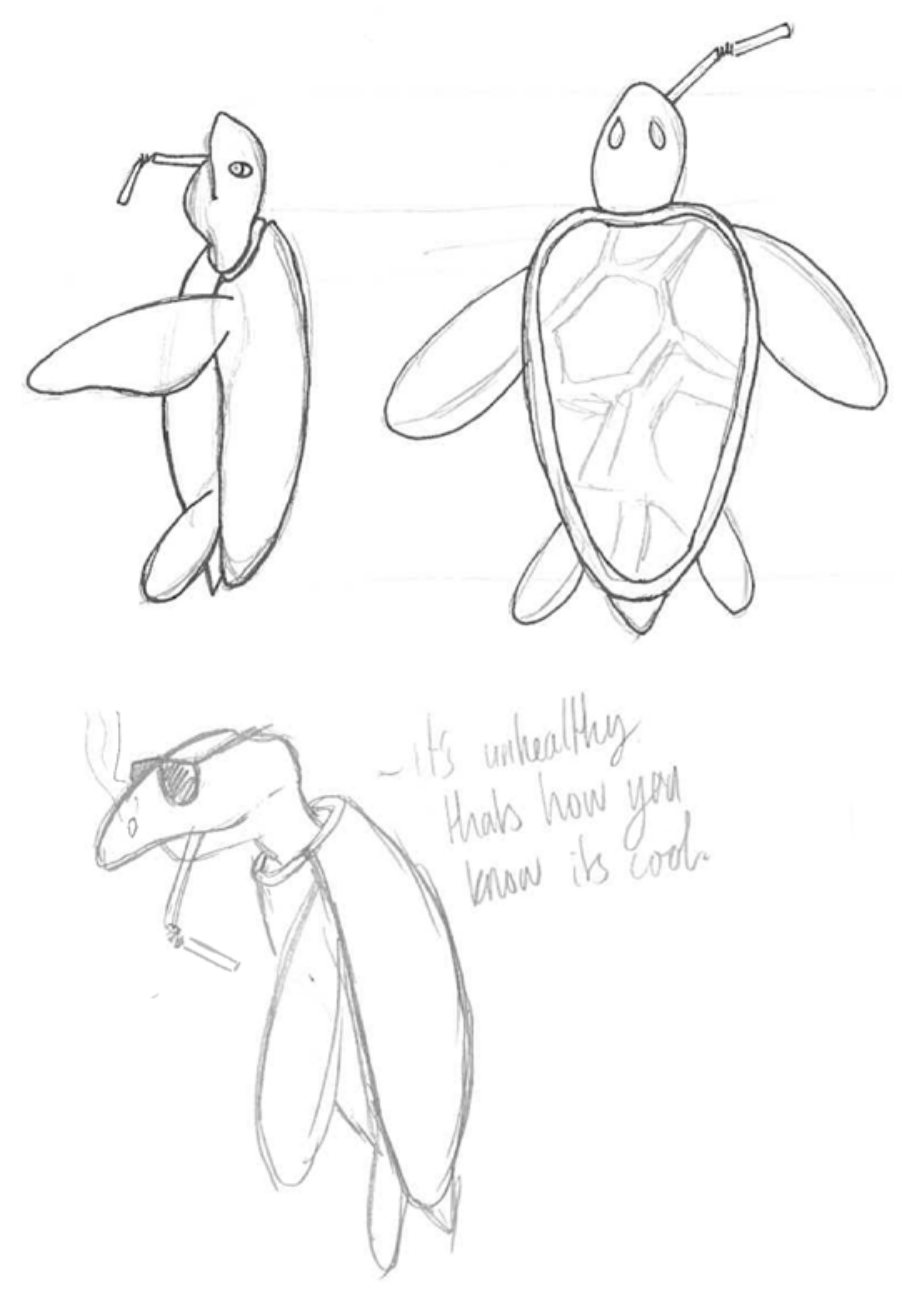

Figure 83: Sketch of turtle concept, orthagraphic drawings used to make 3D model 


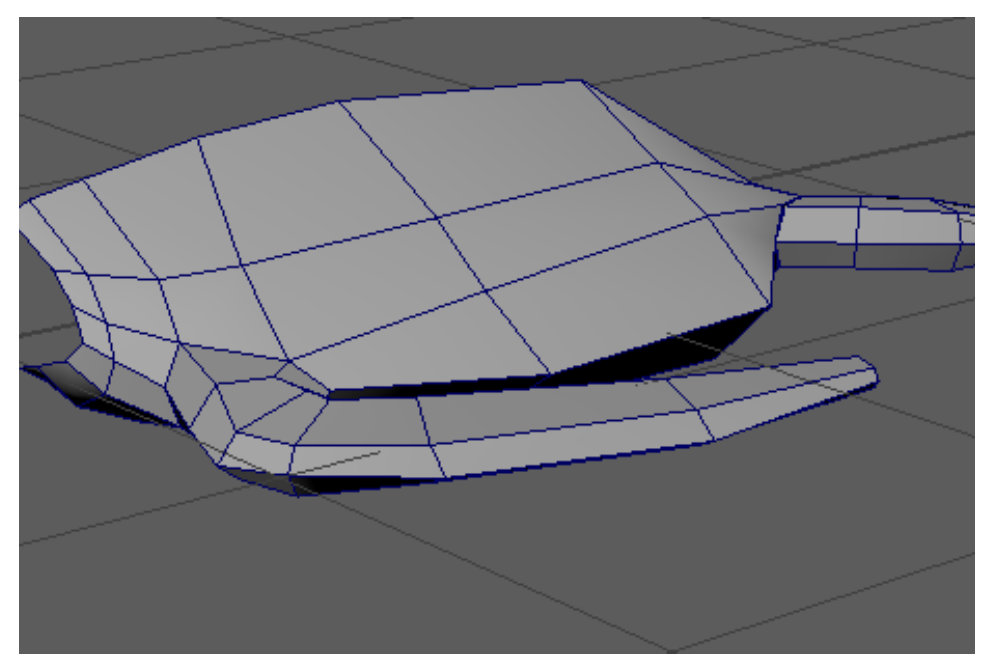

Figure 84: Generating the basic shape of turtle Model

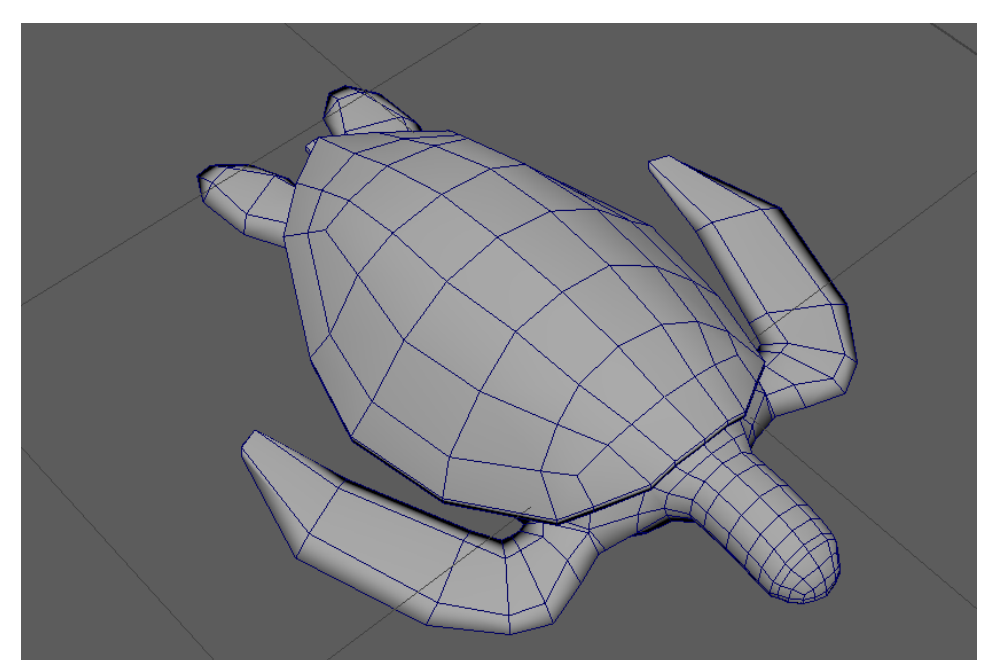

Figure 85: getting the overall form in a low poly mesh

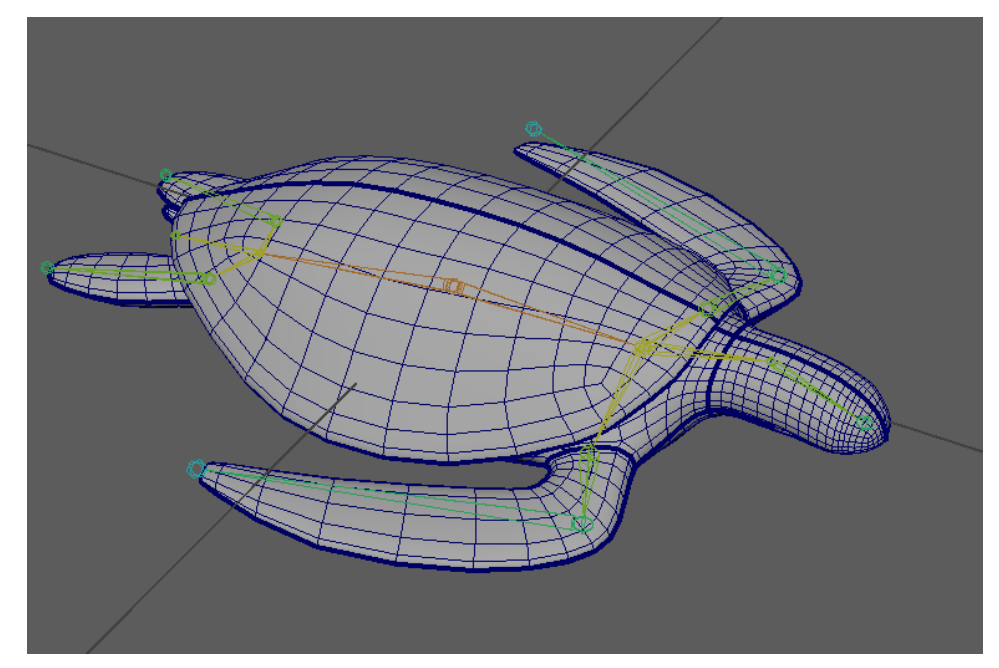

Figure 86: higher poly mesh, rigged and posed.

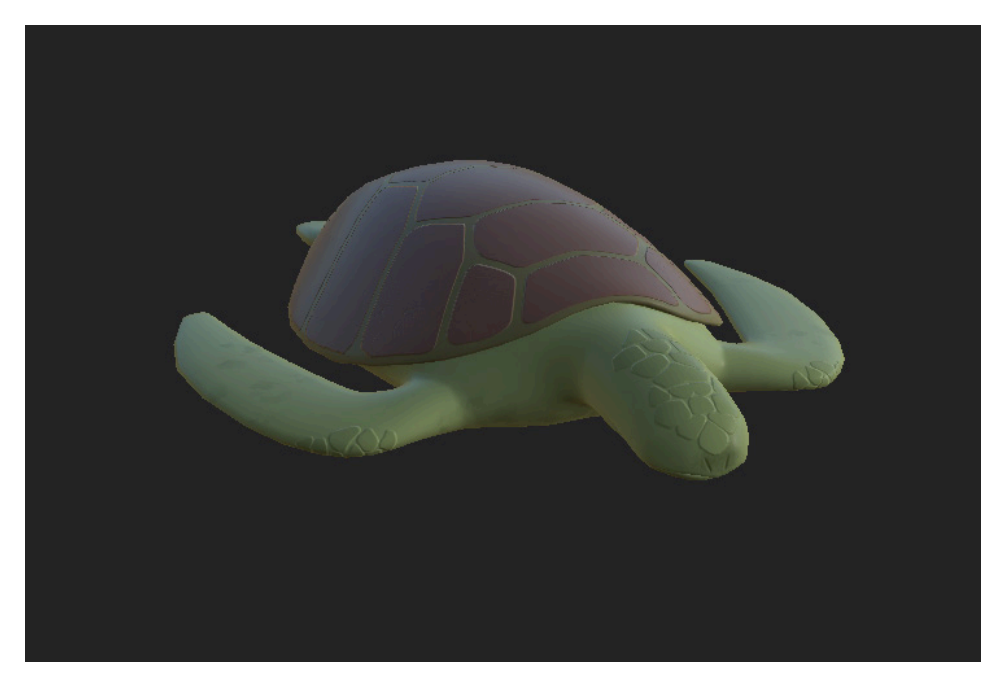

Figure 87: Textures Painted onto the turtle mesh 
To set the scene, the turtle would be swimming through the waste, while still clearly being affected by the straw. For this effect the remaining waste was placed in a unity particle system. This is a system that displays and moves sprites or 3D models in a large number (Unity Technologies, 2018a).
This concept and design was placed in $\mathrm{AR}$, and I felt it was quite successful as an $\mathrm{AR}$ art piece. However, while it worked well as a response to the issue, I felt that it could have made more use of the public space it was located in.

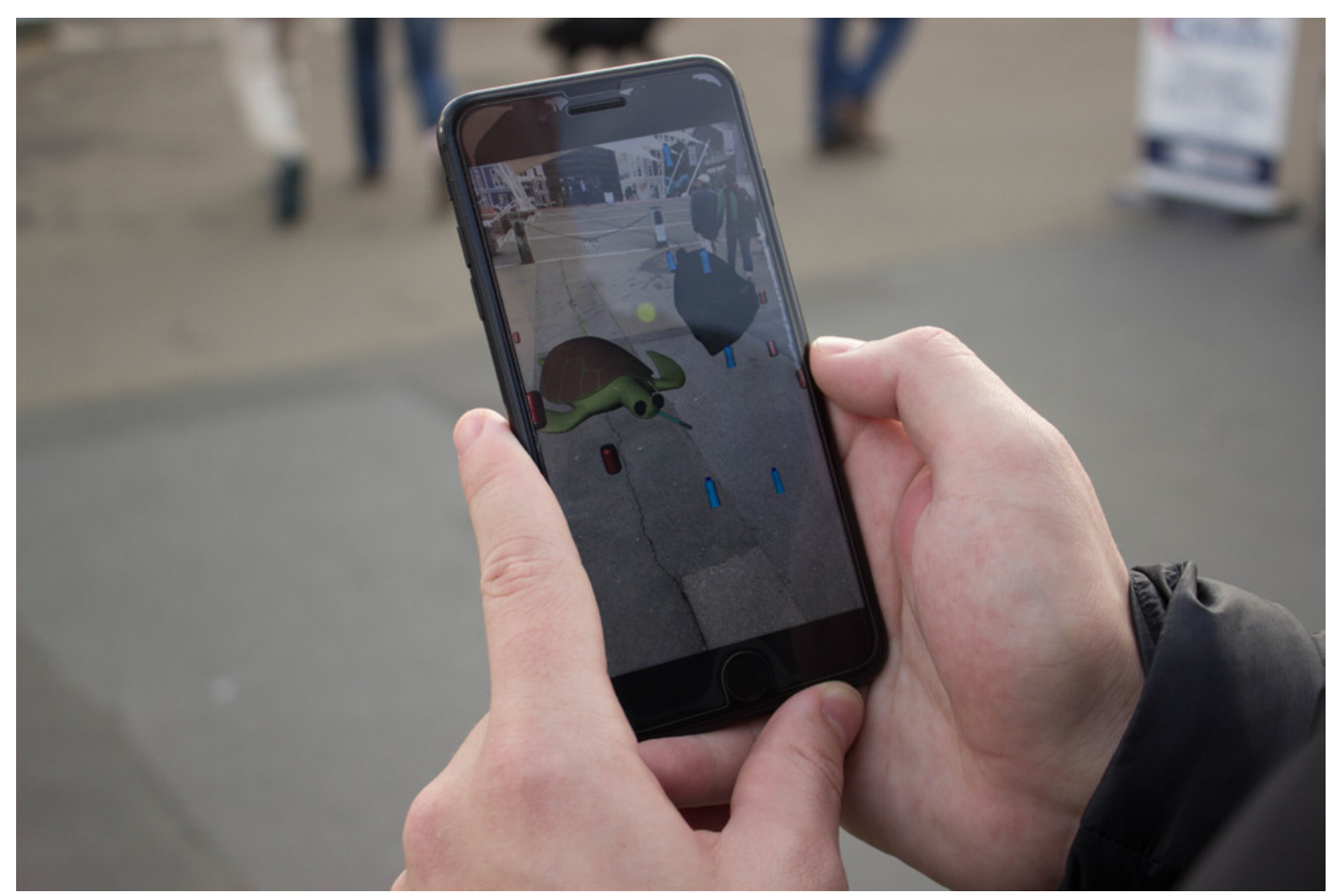

Figure 88: View of the turtle through the device 


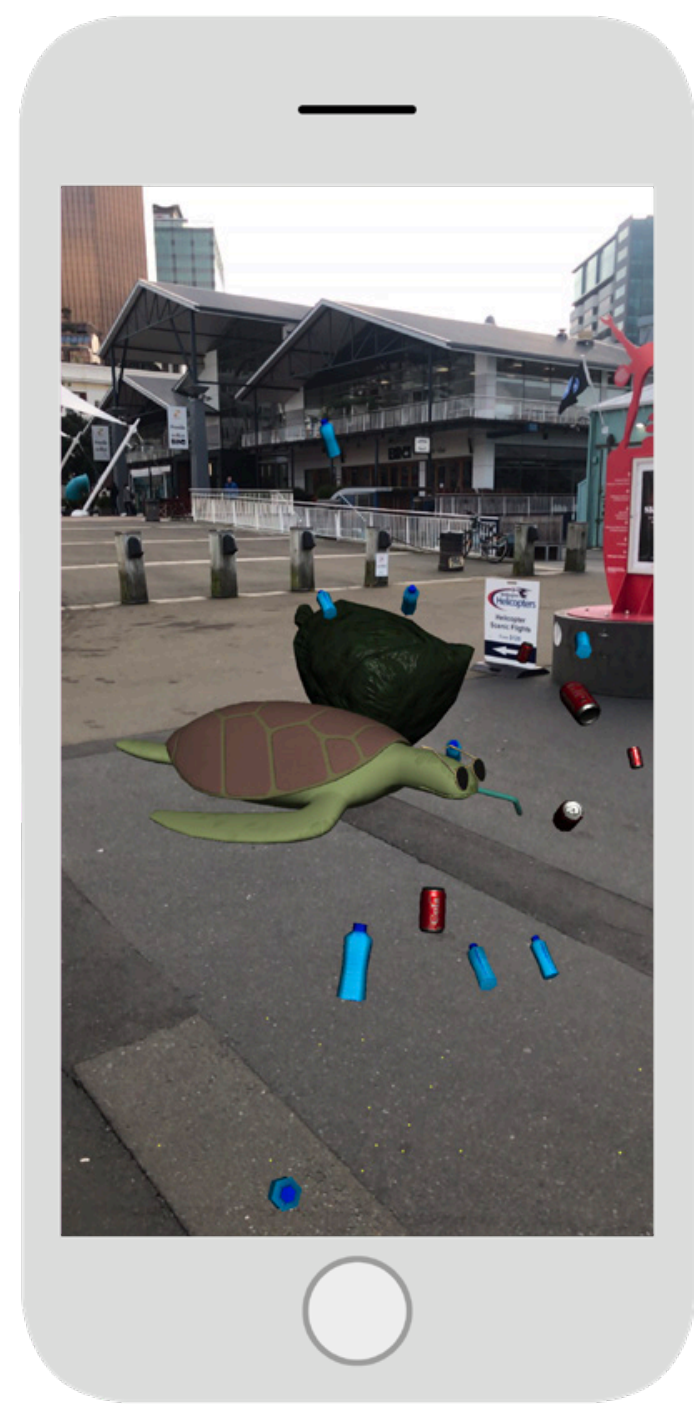

Figure 89: Side on view of turtle in $A R$

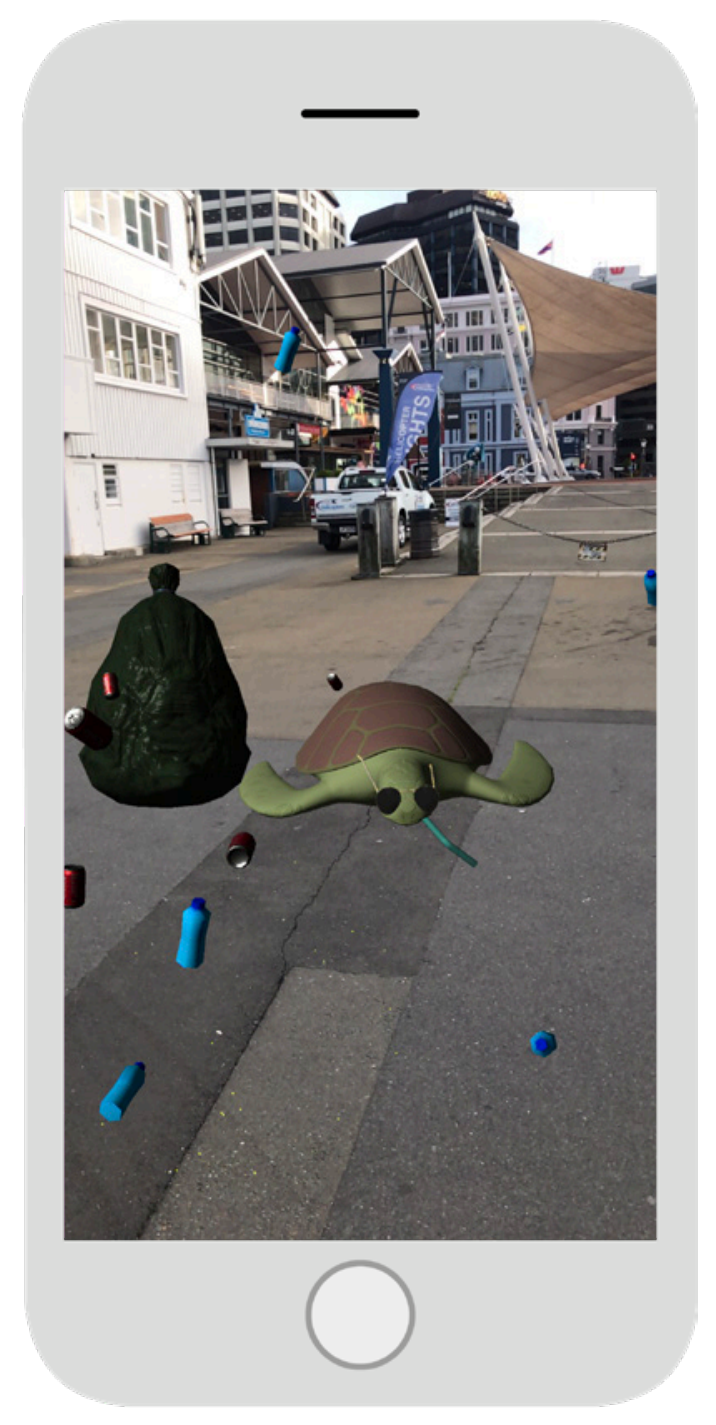

Figure 90: Front on view of turtle in $A R$

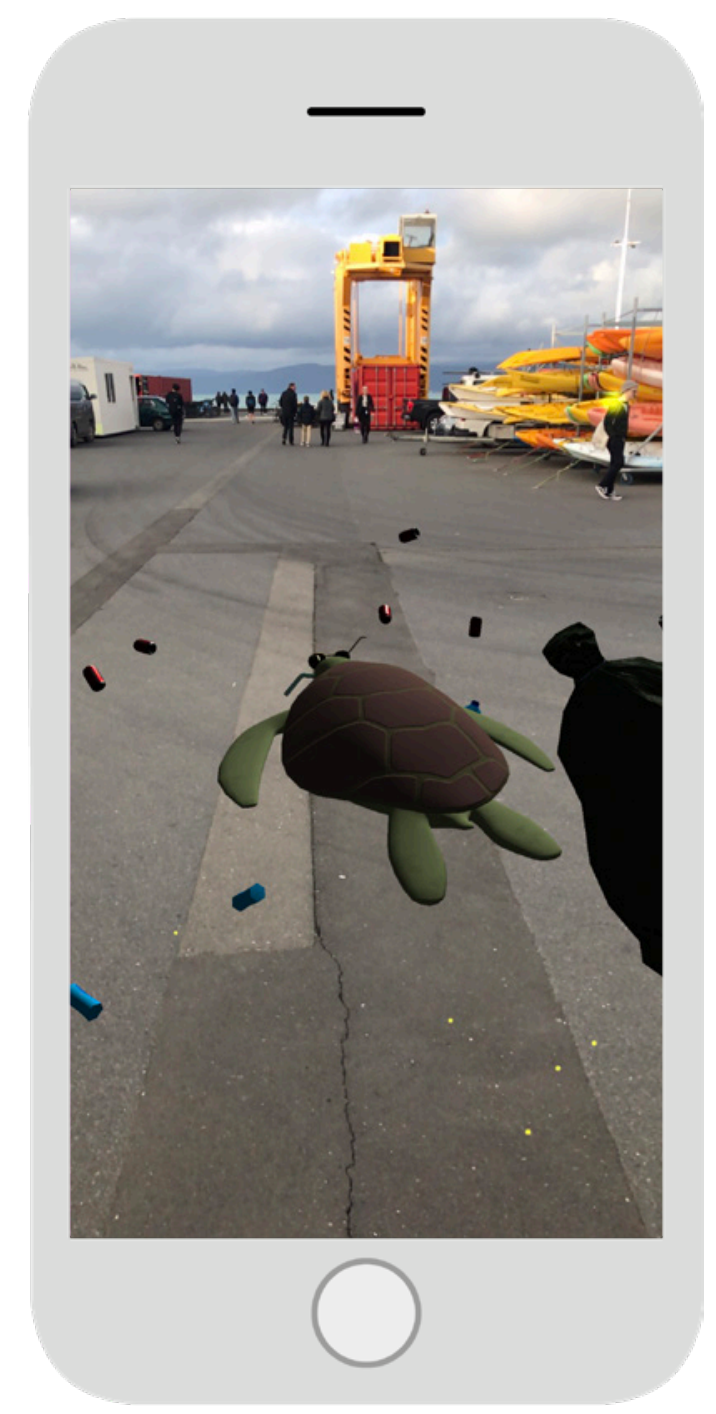

Figure 91: Turtle looking out towards the ocean 
Using the same visual idea and concept, I decided to explore the changing landscape of the issue more. Using AR, a label could be created for places that have removed straws, and for places that have not. These could then update as things change and different places address the issue. This made a more direct use of the context and information of the city, using it as an integral part of its design. As another visual exploration I decided to use a more traditional Street Art style as inspiration, exploring the concept using a stencil-like style, and in heavy line-weights.

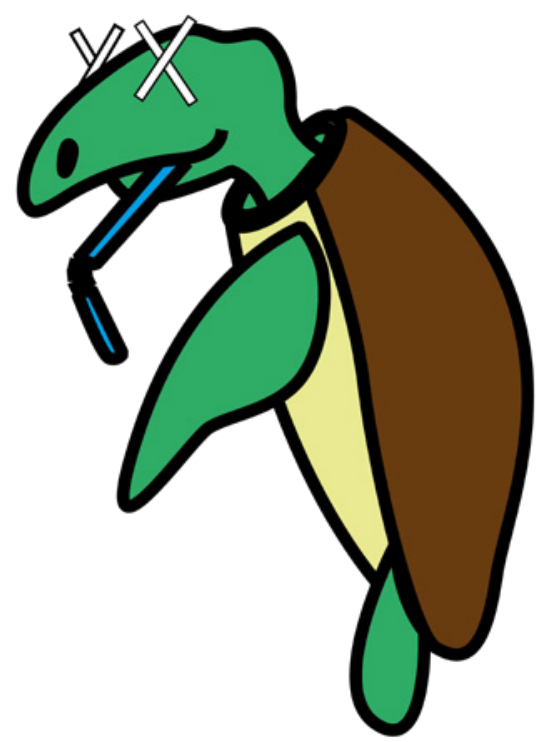

Figure 92: Colour turtle concept with heavy outlines

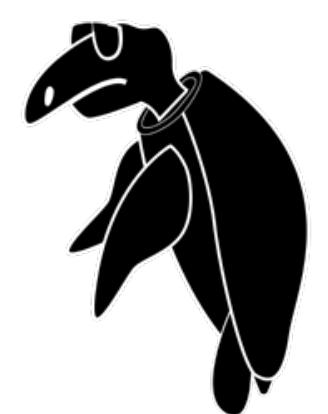

Figure 93 : Initial concpet of a stencil design

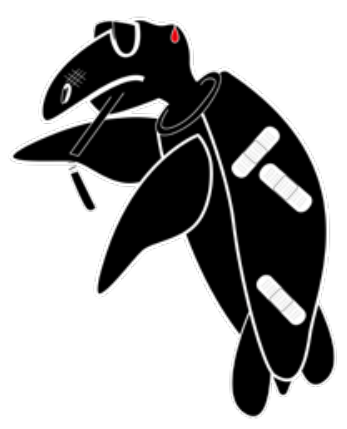

Figure 94: Unwell Turtle concept 


\section{THIS PIACE IS \\ KILLING ME}

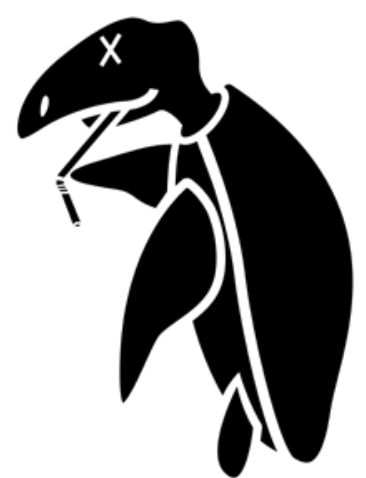

Figure 95: Final unwell turtle design for use in $A R$

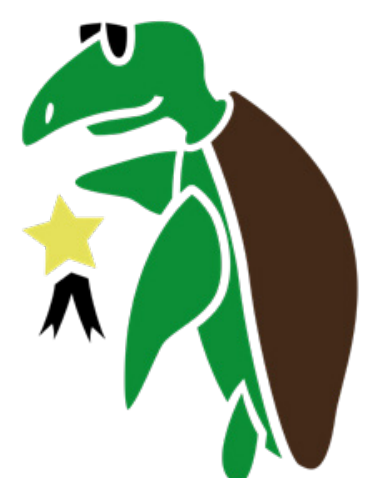

\section{KICKED) 'THE HABIT}

These designs were then placed outside relevant cafes and restaurants in Wellington to serve as an example of how they might be used at a wider scale. The unhealthy turtle was placed outside the local café Midnight Esspresso, which still uses straws. The healthy turtle was placed outside Wagamama on the waterfront, the owners of which have banned straws in all their restaurants.

This concept made more direct use of the specific location it was in and could be used to promote action towards the issue. Cafes and bars could feel a desire and need to remove the damning label from outside their premises. This made the works use their locations to fully inform their design, making use of the street as an artistic resource (Riggle, 2010). 


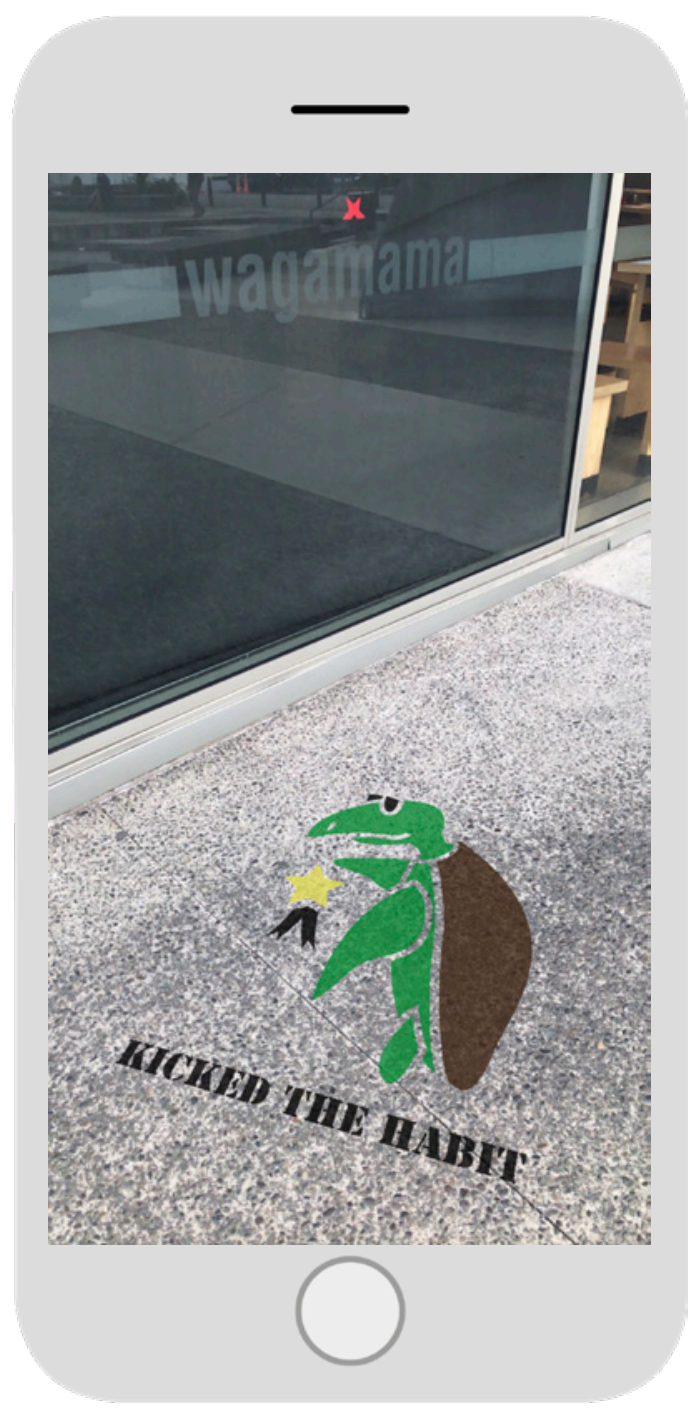

Figure 97: Healthy Turtle in front of Wagamama on the waterfront

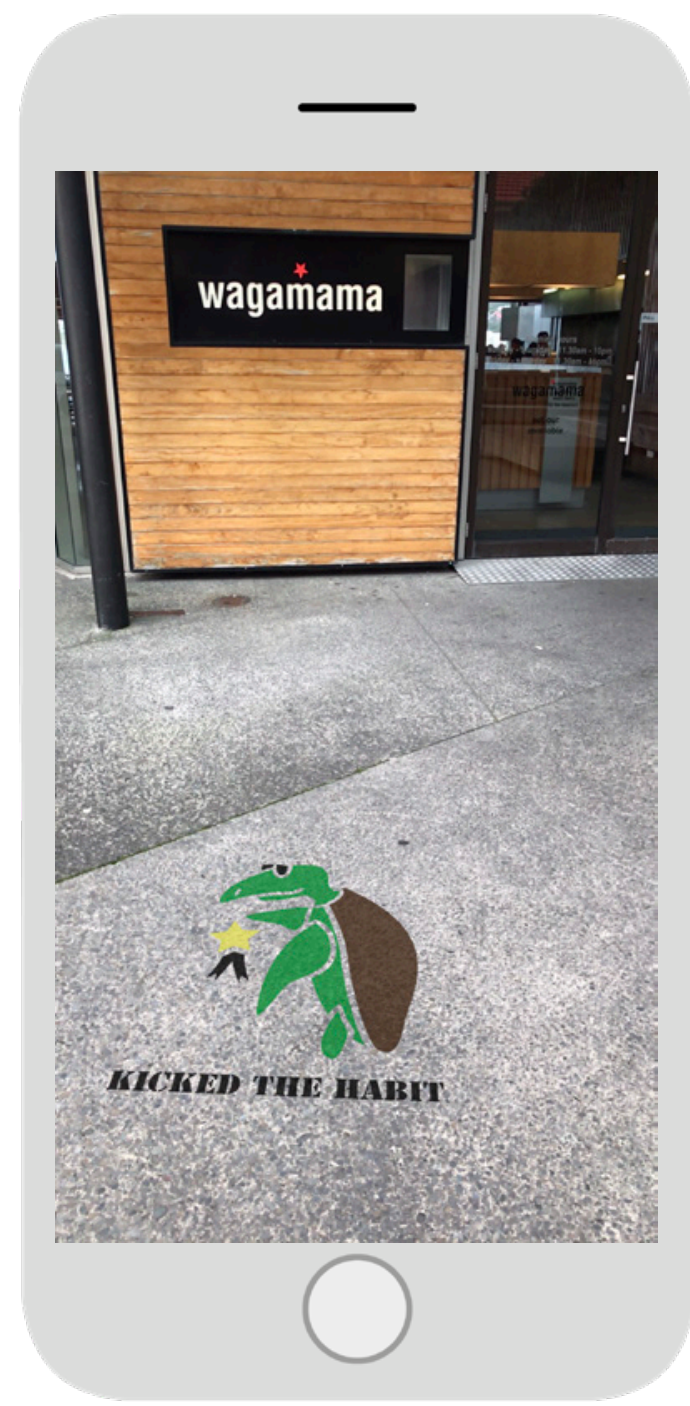

Figure 98: Label reflecting that wagamama has banned straws in all of their restauarants

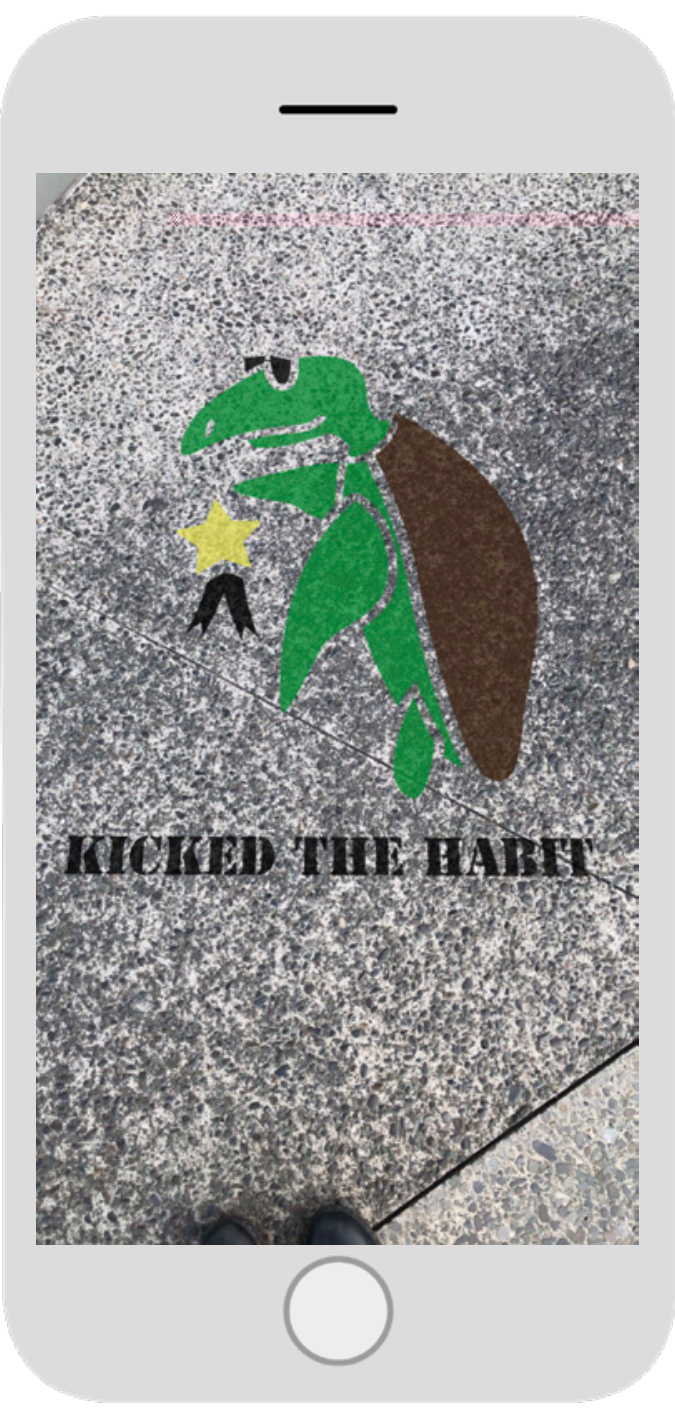

Figure 99: Close up of healthy turtle in $A R$ 


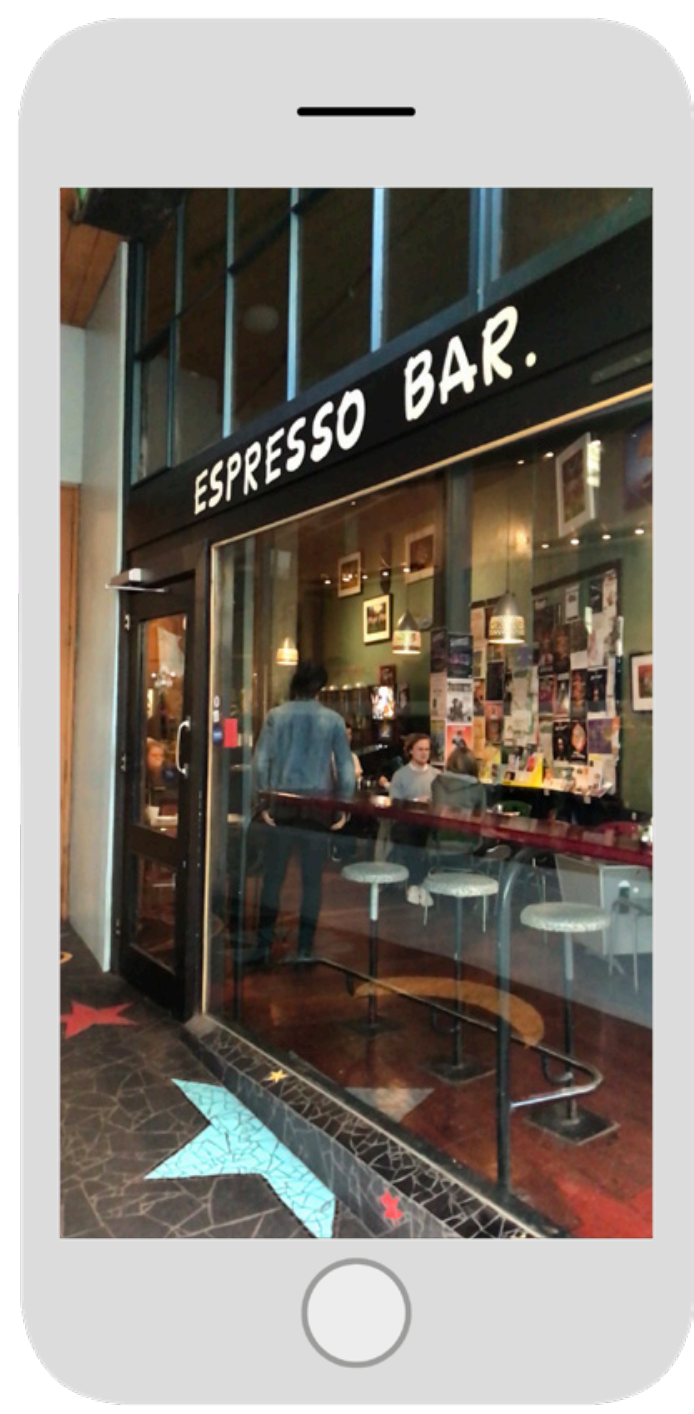

Figure 100: Midnight Cafe in Wellington, still uses plastic straws

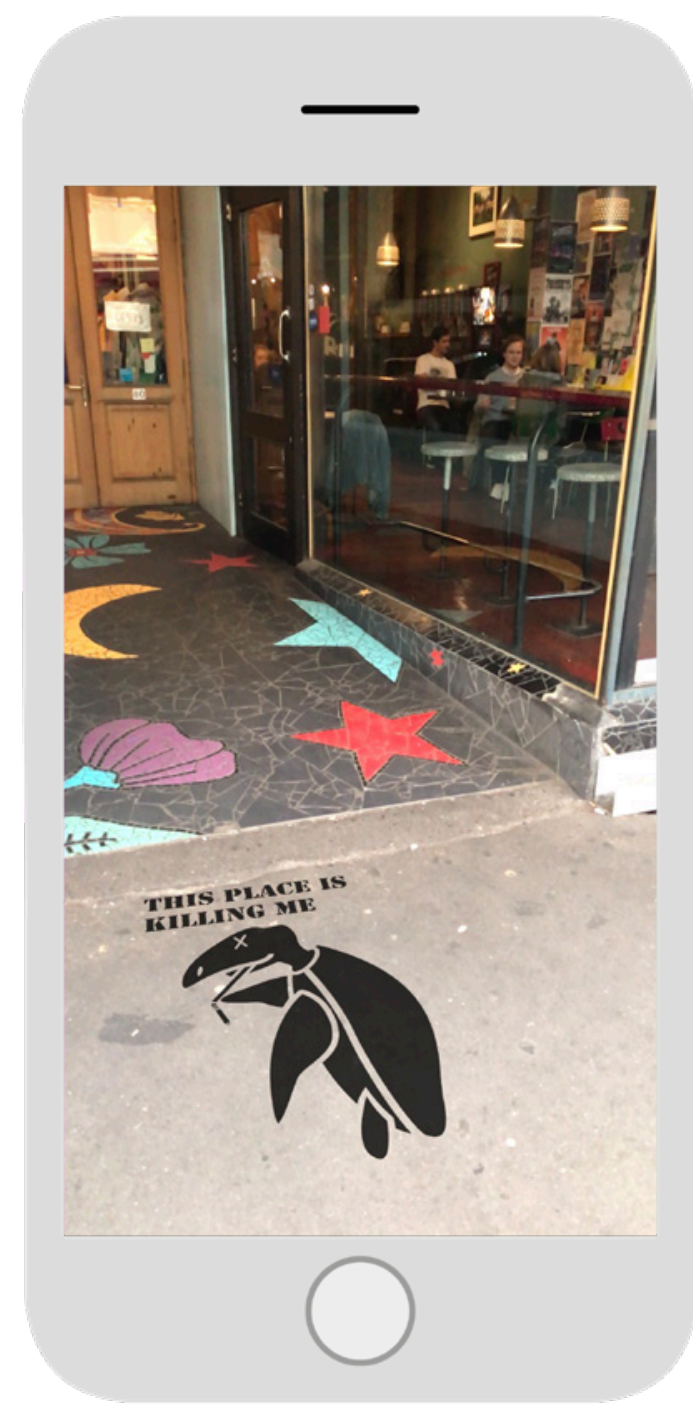

Figure 101: Unbealthy turtle label in front of the cafe

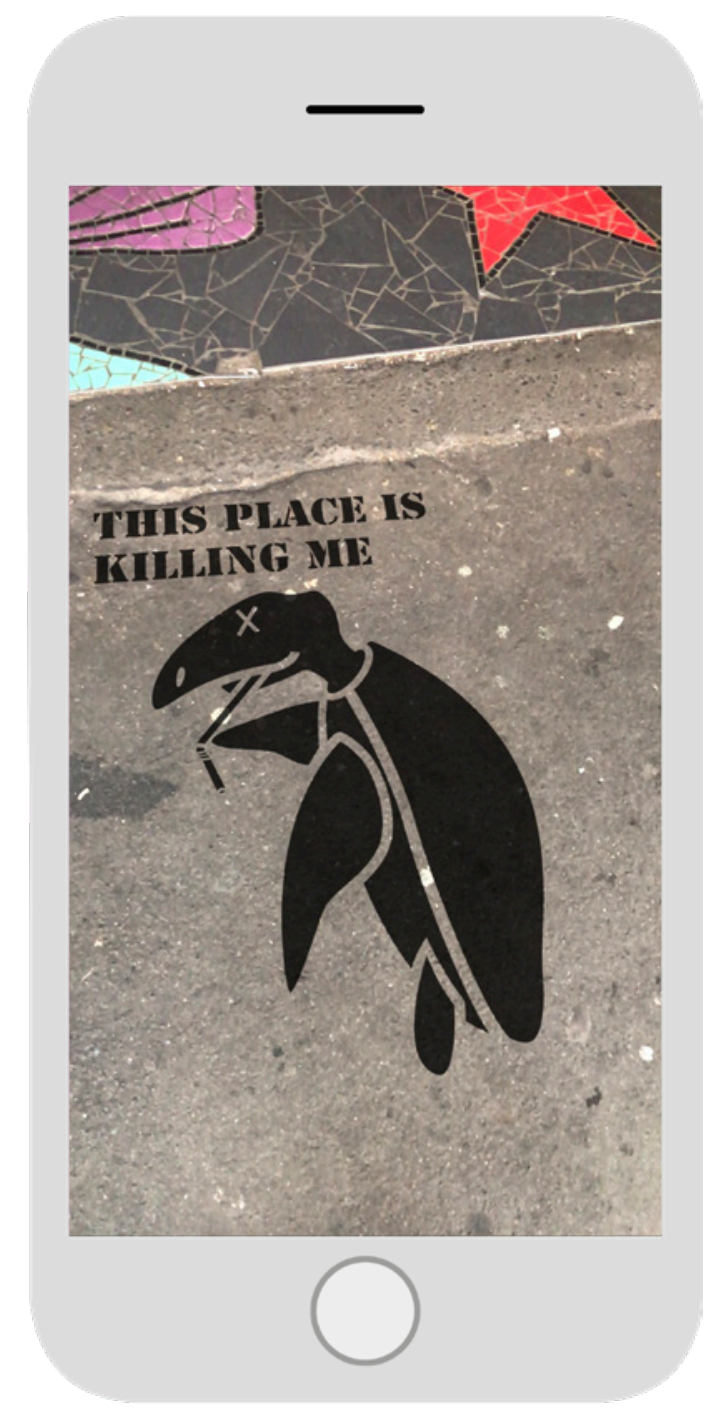

Figure 102: Close up of unbealthy turtle 


$$
\text { 192 }
$$




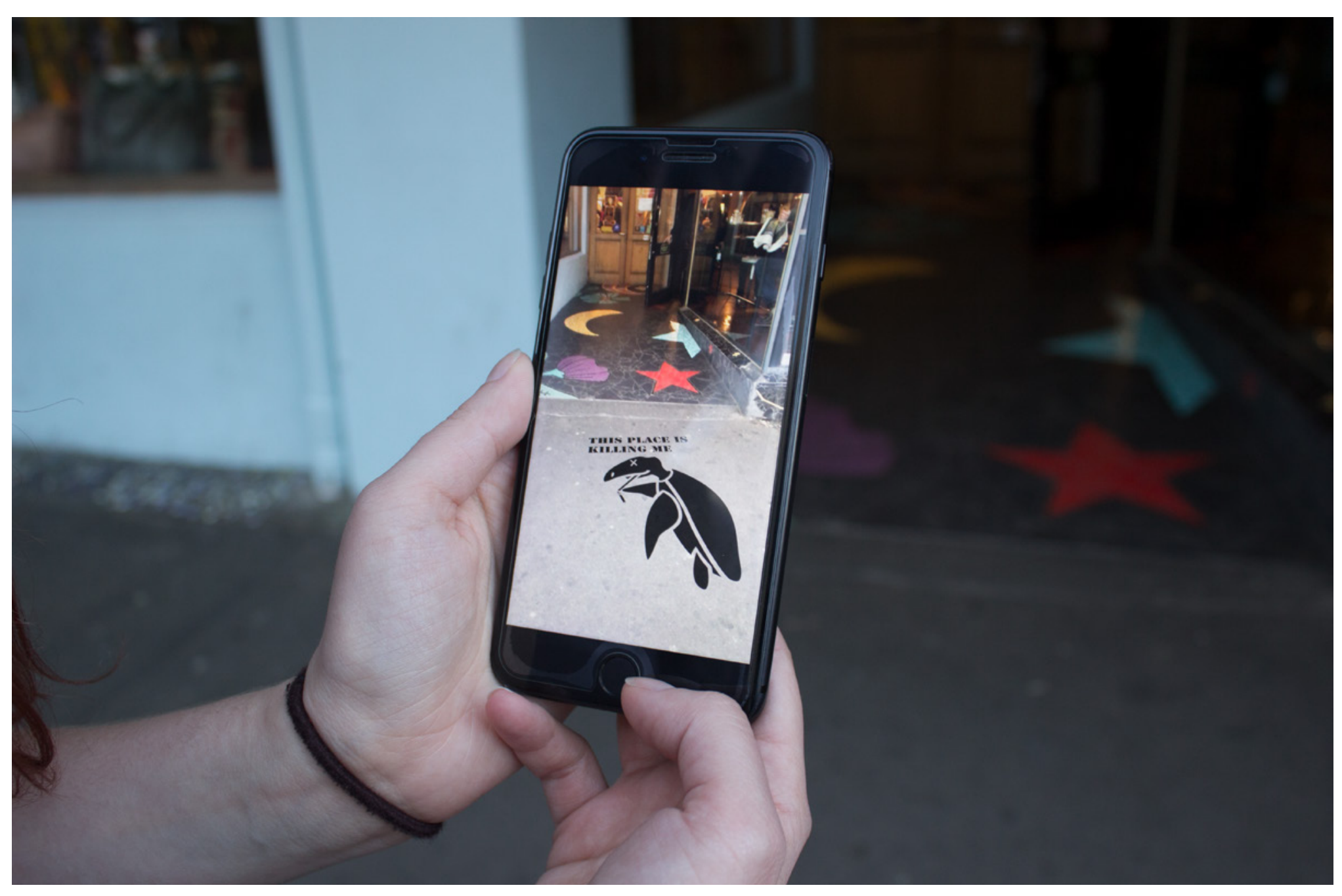

Figure 104: Looking through the device at unhealthy turtle 
During the course of this research, a southern right whale appeared in the Wellington harbour. This was widely welcomed and excited many residents. On social media the creature became an overnight sensation, and they caused several traffic jams.

As much as this public reception was overwhelmingly positive and even accommodating (scheduled events and fireworks were postponed). I did consider what conditions the whale must have endured while located in the harbour, given the plastic waste and potential pollution (Wellington City Council, 2018).

Designs were made to respond to this issue, drawing from some of the earlier research into plastic waste, as well as the presence of the whale itself.

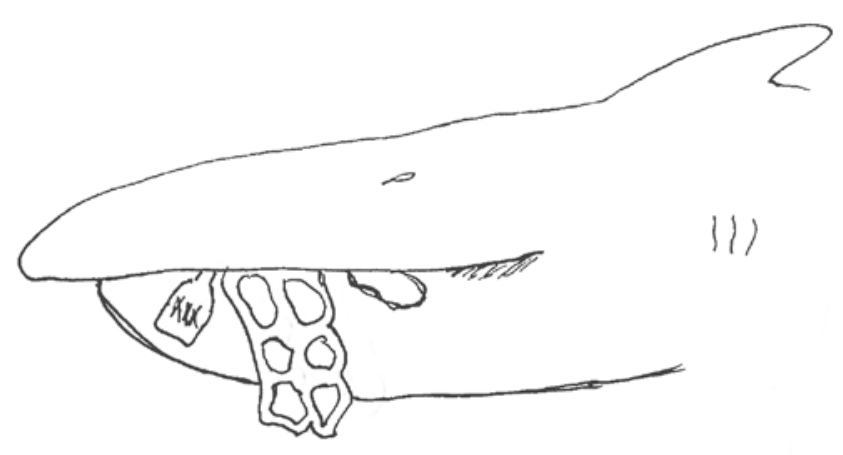

Figure 105: Initial sketch of large sea creature with plastic
The design could be placed on the waterfront, along one of the locations where people had gathered to view the whale. Using this audience and venue, the work could speak to the conditions the whale was more than likely in. In this way I could contrast the outward excitement and welcoming of the whale, with the actual conditions we place it in. The final version of the design used an X-ray to show the inside of the whales stomach and used a comic style speech bubble to give him something to say. This use of humour is not dissimiliar to traditional street art.

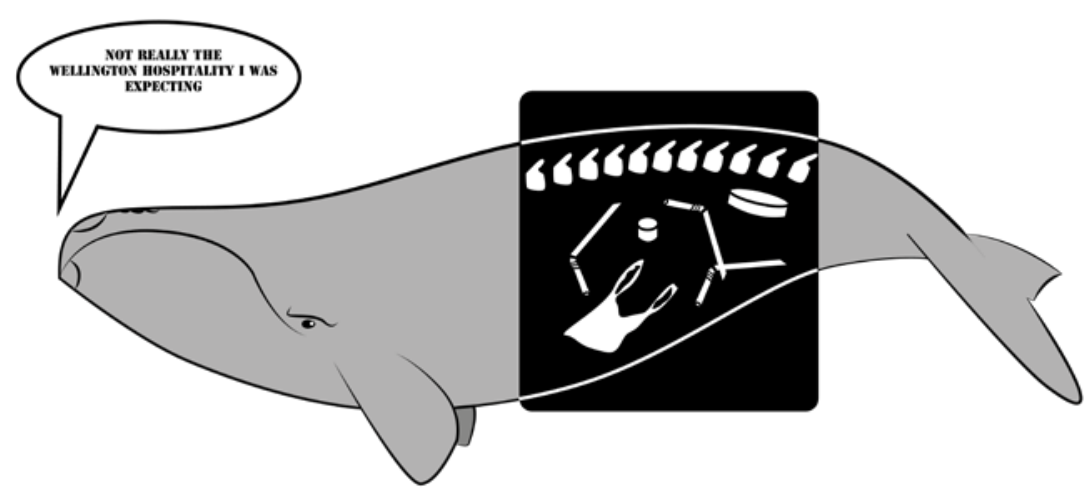




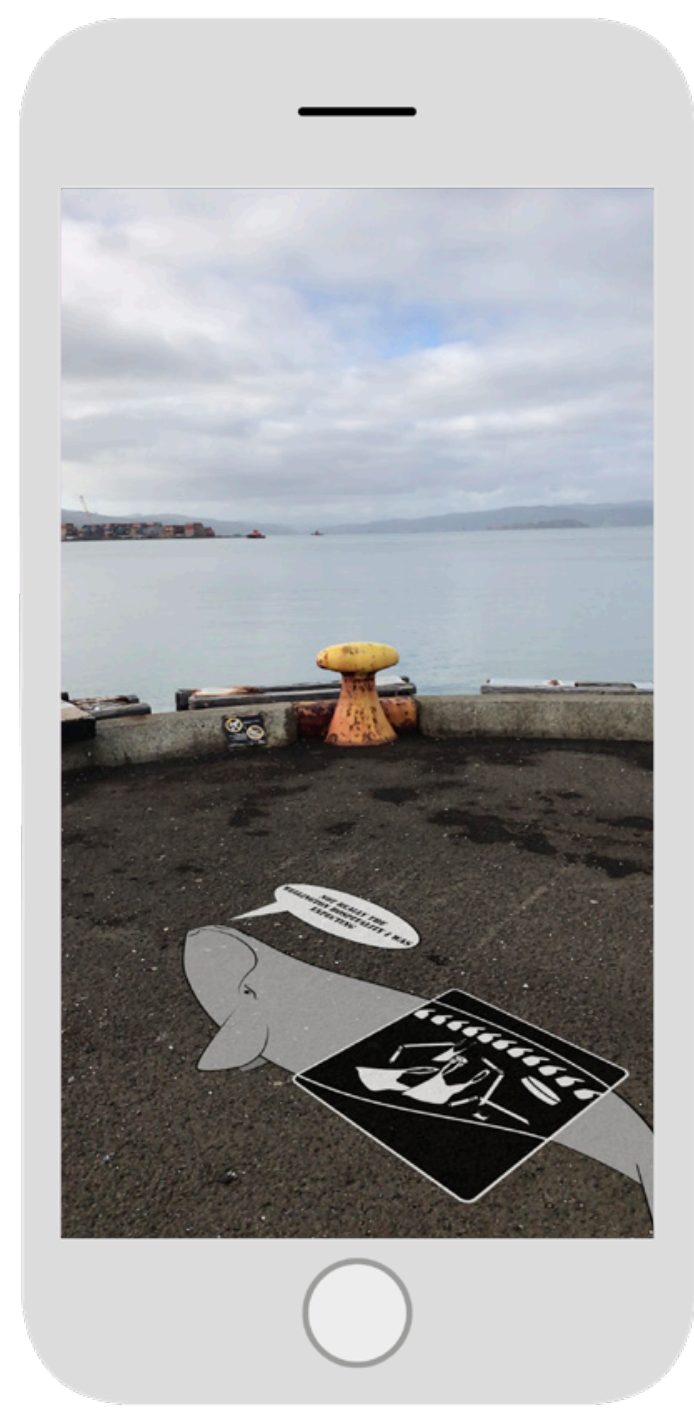

Figure 107: Whale in AR

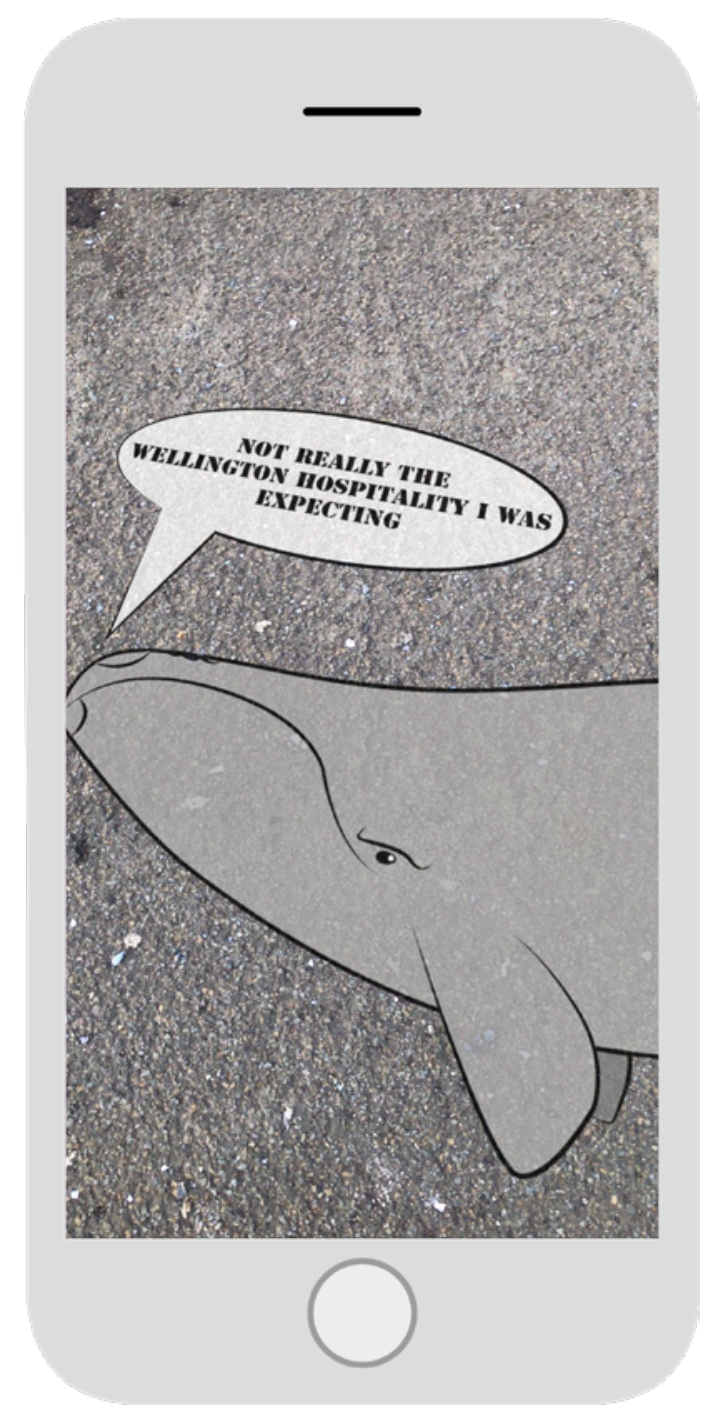

Figure 108: Close up on text element

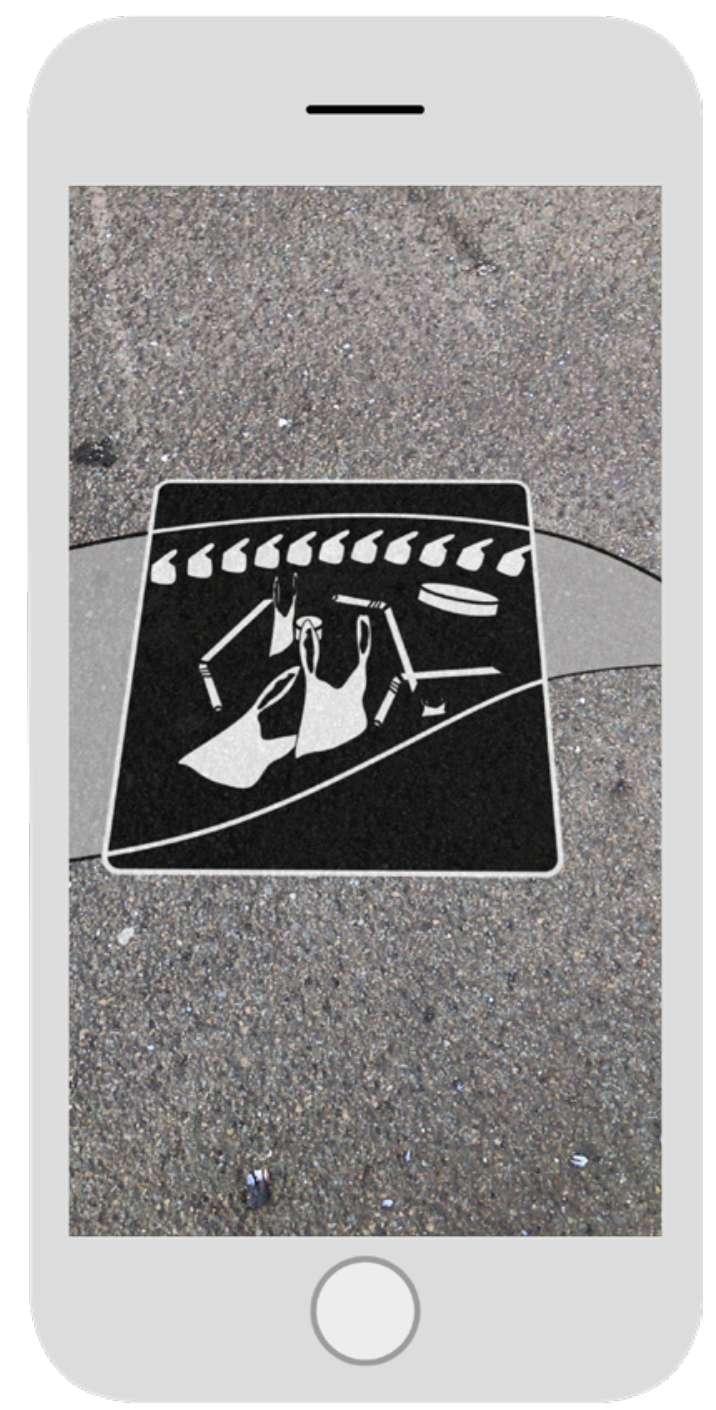

Figure 109: Close up on X-ray 


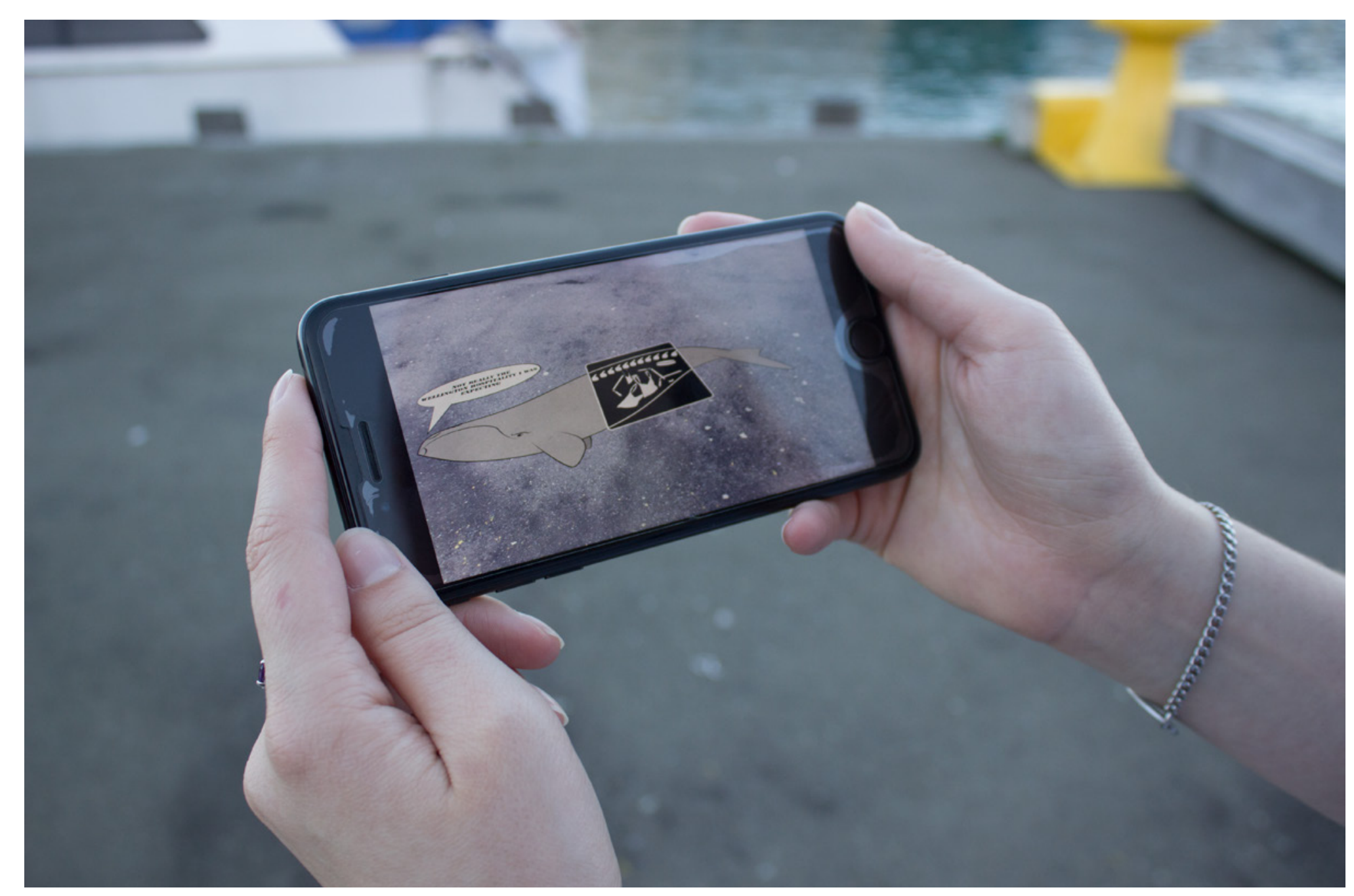

Figure 110: Whale shown through the device 
Both of these designs spoke to the issues of plastic waste in the ocean in a critical manner. These designs were targeting awareness and a shift in perspective; however, I wanted a design that could target an immediate positive response from the casual viewer.

The Public Place Recycling Project is an initiative from the Wellington City Council trialling the placement of recycling stations throughout wellington city ('Public Place Recycling project', 2018). Street Art Designs were then developed with the idea of using these as a site for Digital Street Art.
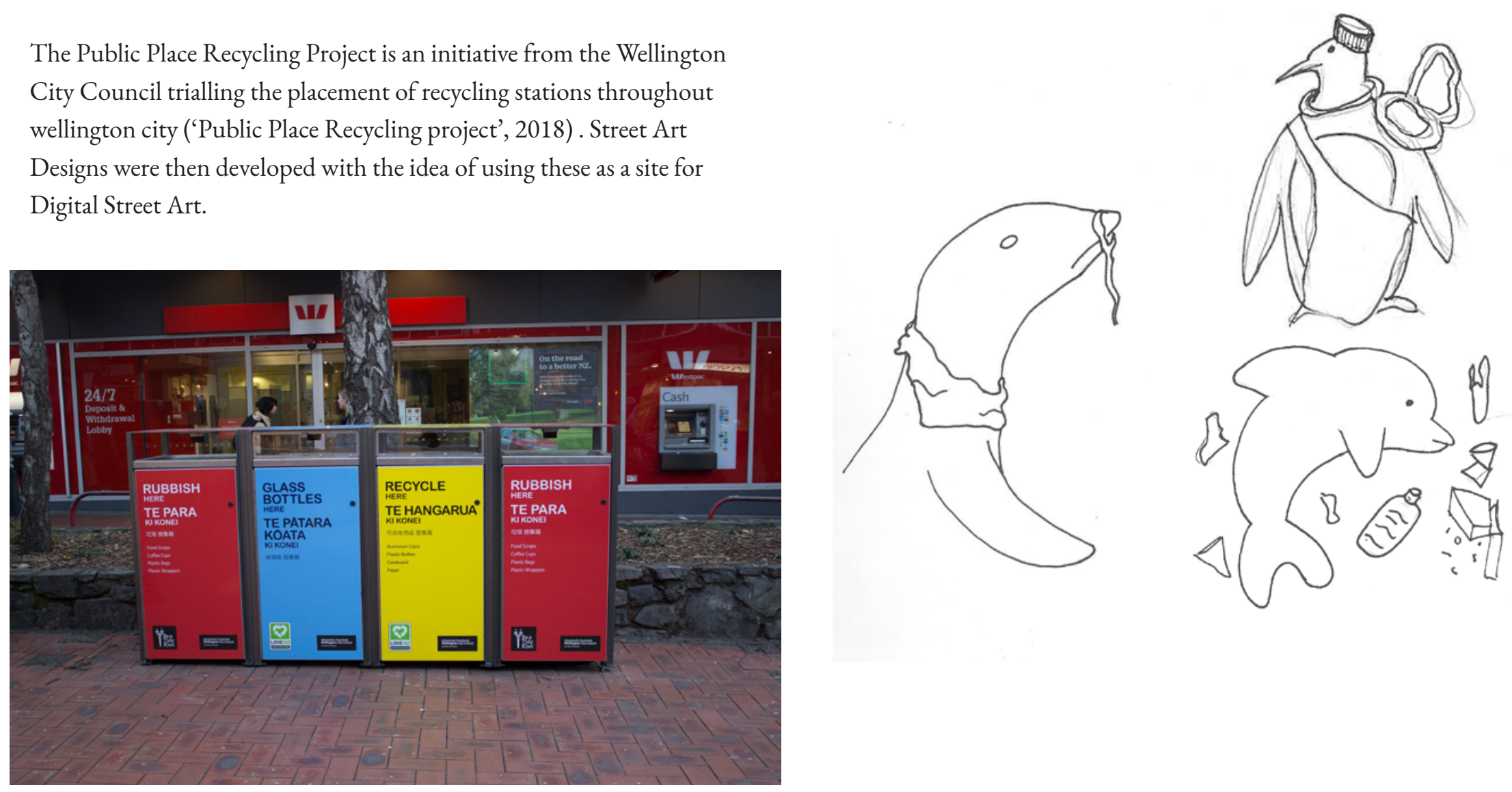
Using the penguin as the character for these designs allowed me to use their cute visual to try and confront and inspire change from viewers. It also worked well due to their size in relation to the recycling stations. These would be accusing towards those that don't recycle, hopefully provoking a response that results in more use of the recycling bins. The final visuals made use of the stencil style used in the turtle concept, using very simple visuals in contrast to the busy visuals of the recycle bins themselves.

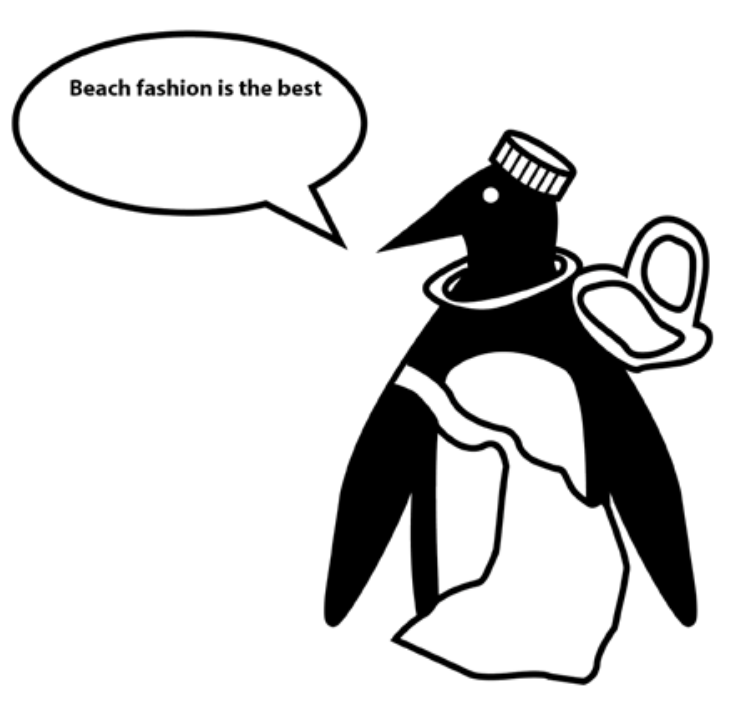

Figure 113: Development of penguin as a graphic

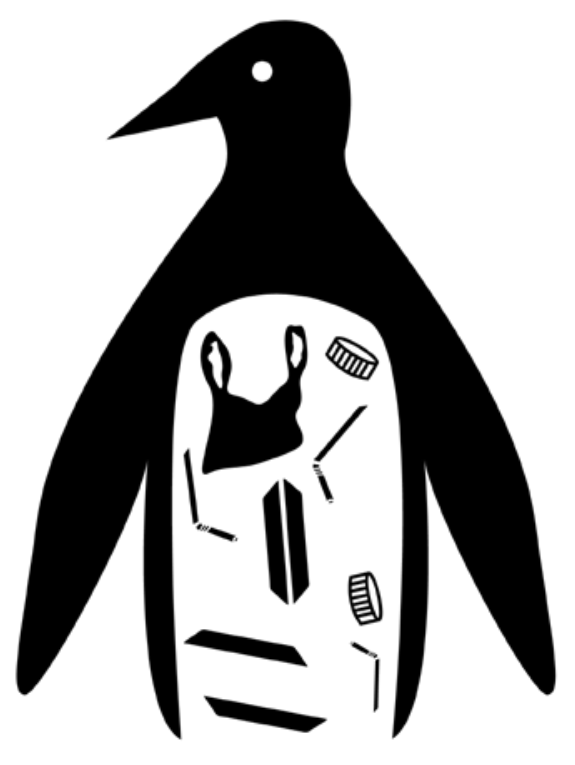

FEND) 'THE BINS NOT ME

Figure 114: Refined penguin design, using a more stencil design

86 


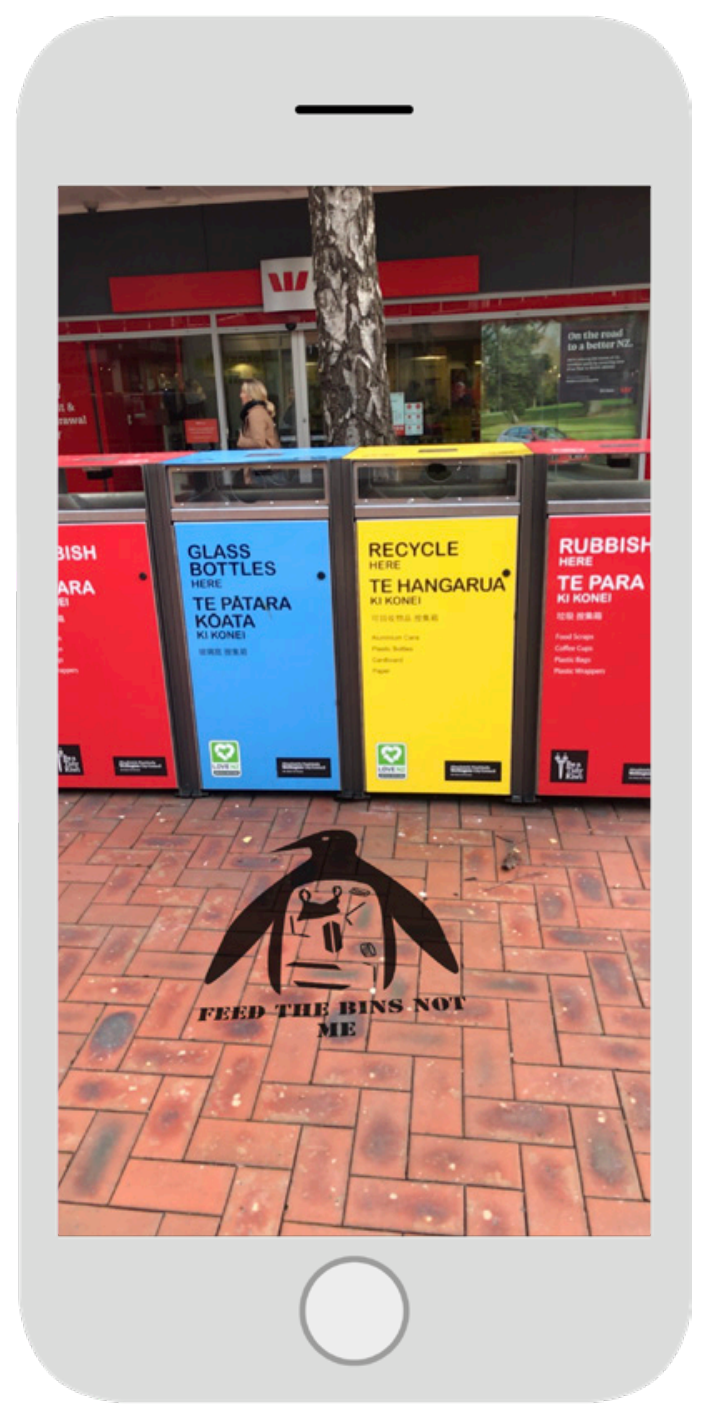

Figure 115: Penguin stencil in AR

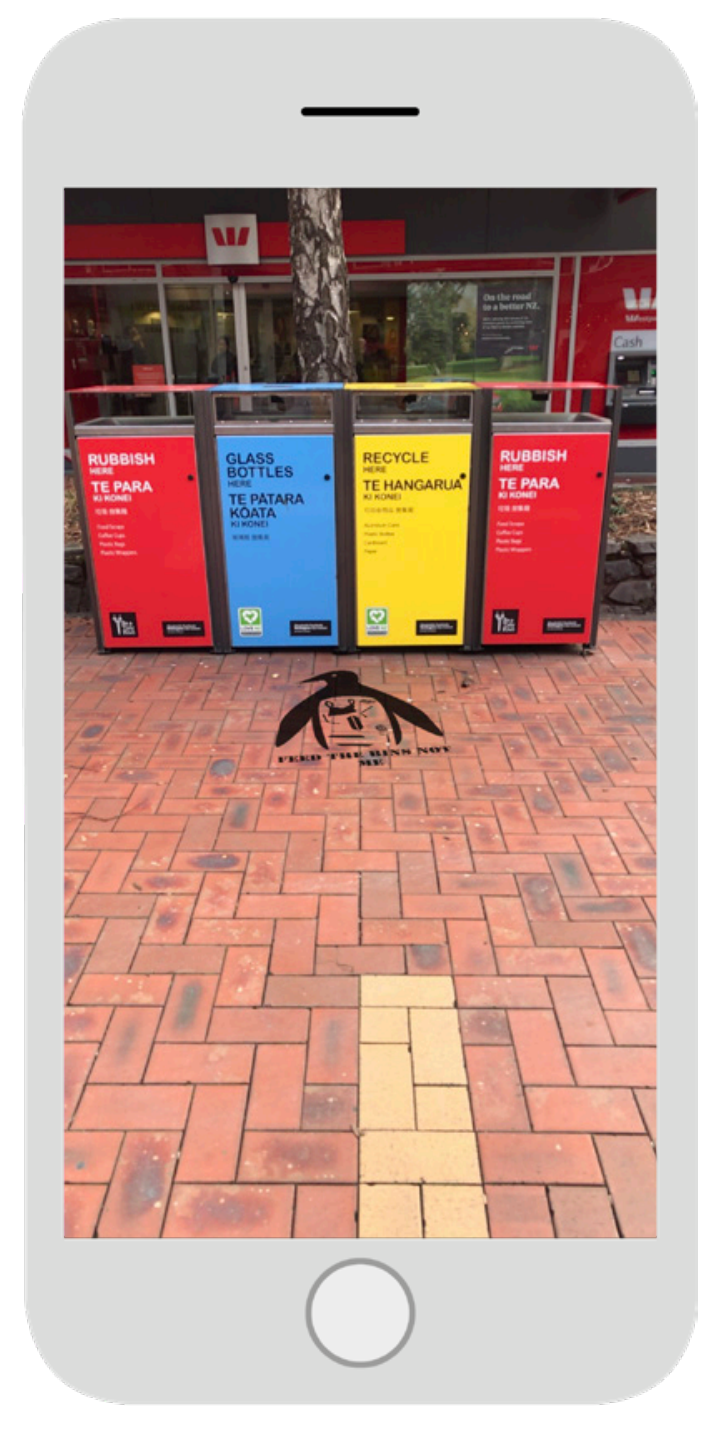

Figure 116: Penguin framed with the bins

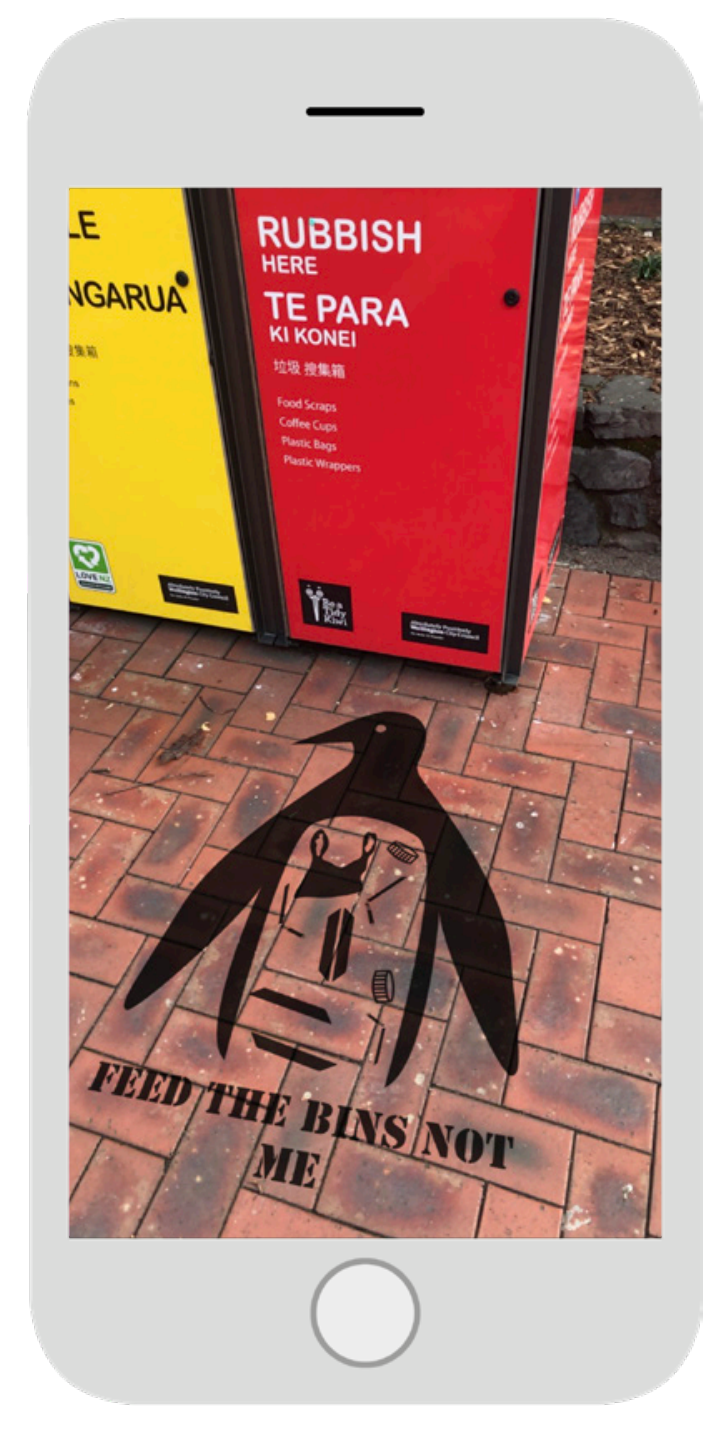

Figure 117: Close up of penguin 


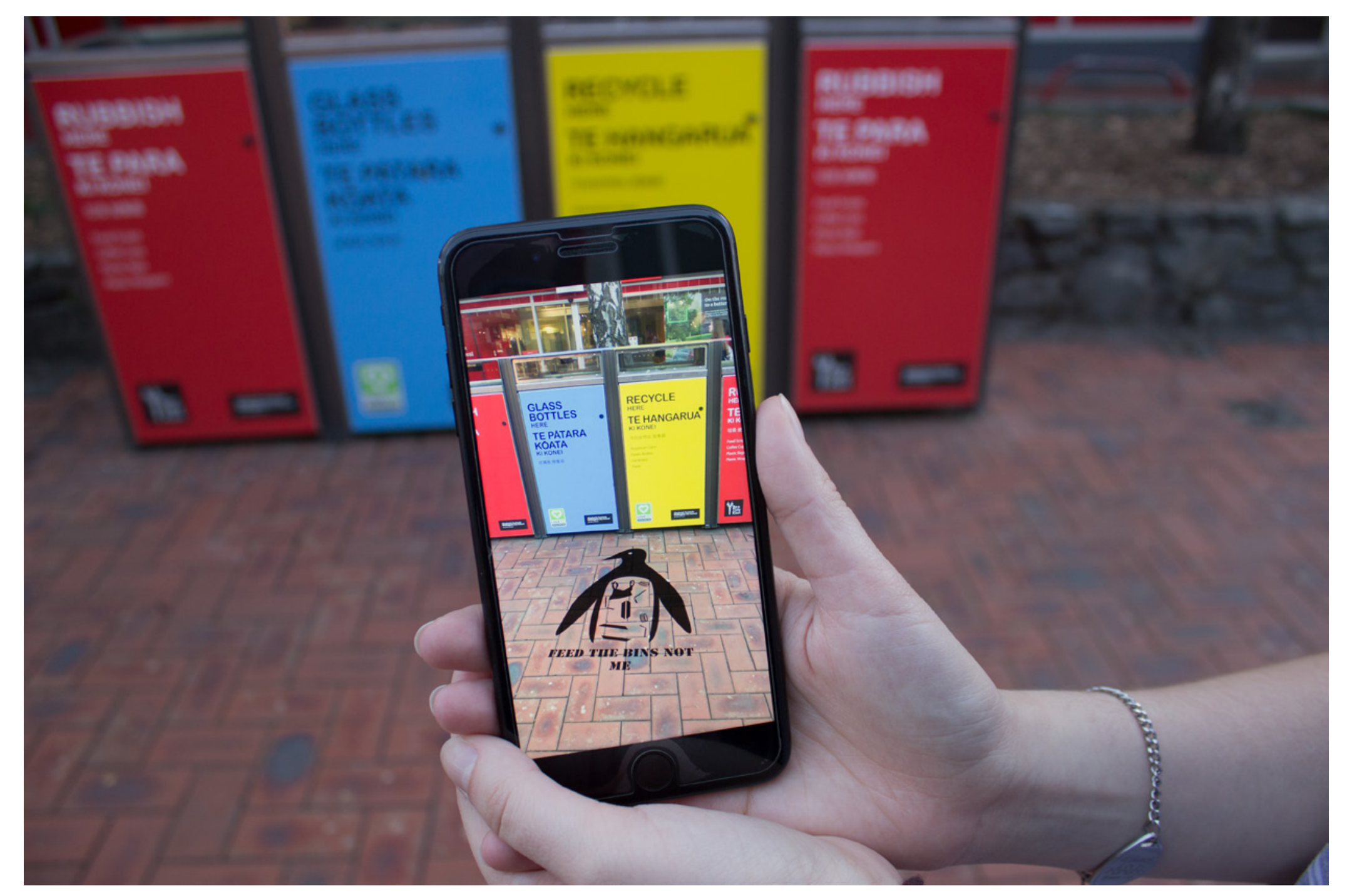

Figure 118: Penguin shown through the device 


\section{Reflection:}

The outputs of this scenario aimed to address a core environmental issue, that of single-use, disposable plastic and its effect on marine life. Using specific issues and events to talk to the wider issue, these works drew direct context from locations and events throughout the city, using the public space to provoke a response. This scenario developed into outputs that spoke to local current events and changes throughout the city. Using Augmented Reality allowed the works of Digital Street Art to be updated, referencing the context of the location, even when things change. These works made direct use of their location on the street to inform their designs, and their meaning would be severely lessened when viewed away from these locations. This results in these designs being of the street and using it as an artistic resource, informing its nature as Street Art (Riggle, 2010).

\section{Further development and considera- tions:}

Developing this scenario further could have tied the different designs to live data and display. For example, changing the turtle labels based on news and data about the places they were in. More interactions in the designs could have led to more engagement with the issue, for example the whale could have been made interactable with a donate button. This would have used the attention and conversation sparked by the physical whale and the Street Art response to generate income for the organisations trying to improve the marine life.

Further developments in technology could also allow for expansions in these designs, for example a future version could recognise the actions of the viewers around them and act accordingly. For example, the penguins could berate those that don't use the bins. 


\section{Discussion}


This research focused on the creation of Digital Street Art and how that could be created using Augmented Reality (AR). The fields of AR and Street Art research were investigated and compared. This showed strong parallels between the two areas, and opened the new avenue of Digital Street Art as a means for creative expression through AR.

AR content allows for digital expression within a physical space and is strengthened by a direct relationship to that environment. AR provides a number of features and can be used by artists in a number of ways: Digital content can be animated and interactable (Billinghurst et al., 2001).

Using AR, this content could be created at a large physical scale (Pacheco et al., 2015) or be placed in locations where physically generated content would be difficult or impossible (Skwarek, 2011, 2014). The content could supplement real world objects with information or entertainment (Ajanki et al., 2011; Billinghurst et al., 2001). Using these possibilities, AR could dialogue with the physical world, commenting on and responding to scenarios. This could be used to reclaim public visual space, as well as bring attention to public issues, concerns and perspectives, with the aim of provoking a response.

Street Art is a highly creative practice independent of the artworld (Riggle, 2010), responding and drawing context instead from the world around it. Street Art uses the street as integral part of its meaning, informing its design and being used as an artistic resource (Riggle, 2010).This can be in a materialistic or contextual way, using either the physical features or social contexts of the location. Street Art has come a long way from the graffiti that inspired it, now often commissioned and condoned. Many now see
Street Art as a highly creative urban art form (Rowe \& Hutton, 2012) and this popularity has only increased the scale and quality of the Street Art created. Street Art can use its public nature to bring attention to issues and comment or respond to social, political, economic and environmental issues (Sarah Banet-Weiser, 2012).

Digital Street Art can therefore use the strengths of AR technology, and the ideologies and concepts of Street Art to create digital content with a strong and direct tie to the physical world. It can confront that world, comment on or add to it, provoking responses from those that view it.

The contextual research of this thesis focused on the histories and development of AR and Street Art to inform the design practice of creating Digital Street Art and how that could be distributed. More literature relating to platform economics and the digital age could have strengthened the supplementary argument of a platform for Digital Street Art.

As discussed in Chapter 2, mobile AR is currently the most popular and prevalent method of AR. This is largely due to its use of a device that is already prevalent within the population. This makes it the ideal method of AR when considering Digital Street Art, which should be as democratic as possible. This is in line with both Street Art and AR art practice, which challenge the boundaries of the traditional artworld to exist outside of it. The democracy of this Digital Street Art would be increased with a platform allowing creators to upload Street Art for consumers to view. This platform would be open and accessible to anyone with access to a 
smart device, allowing users to view digital Street Art at the locations for which they are created. Before discussing the specifics of such a platform, its content would need to be considered in more detail, hence the focus of this thesis being on Digital Street Art itself.

For this content to be developed, the technology and precedents were explored, allowing this research to frame the possibilities of the technology and seek inspiration for designs. Experimenting with the available technology allowed for a deeper understanding of the technology with relation to the proposed output. This was complemented by exploring precedent works using AR technology, as well as Street Art within the city. Exploring Wellington supplemented the knowledge used to inform final designed Digital Street Art for locations within the city.

This exploration of technology and space was limited in a number of ways. Only Mobile ARKit on an Apple device was used throughout this thesis. Although the insights gained could still be applied to other hardware and software combinations. This research was also limited to locations within Wellington, New Zealand, other locations could have different implications for generating AR content. The research did not consider high fidelity, complex 3D models as part of its technical exploration. This was, in part, due to my own skills in this area being limited, and also due to the accessibility of this skillset and technology to the wider public. Accessibility and ease of access was a recurring theme throughout this research and the final designs were always going to reflect that in their technicality. It is for this reason that photorealistic rendering and modelling was not considered during the free experimentation phase.
From this experimentation, two scenarios for Digital Street Art were developed, targeting key issues in Wellington city. Each scenario allowed for multiple designed responses, addressing the issues in different ways with the aim of provoking and eliciting response. The issues addressed were the rental crisis in Wellington and plastic waste's effect on the environment, showing the possibility to address both economic and environmental issues. The pieces created were designed to draw direct context from the physical location in which they were found. Viewing these pieces away from these locations would be possible however their meaning would change, and the provoked response would be lessened.

These outputs prove that Digital Street Art, while making use of a new technology, still represent Street Art practice due to their integral use of the public space they were in as an artistic resource (Riggle, 2010). Each of the designed outputs spoke to important issues, something that modern Street Art is very familiar with (Sarah Banet-Weiser, 2012). These designs would exist outside of the artworld, dialoguing with the public instead (Riggle, 2010).

The use of technology does impose some restriction in use and availability. However, the prevalence of the mobile phone in today's society does reduce this restriction and allow it to be in the public space. To reduce this limitation further, tools for local content creation could be considered in a platform application for Digital Street Art. Another limitation is mobile content being tied to the companies that provide these devices, even indirectly. This limitation could be addressed by further research 
into the platform designed. For example, the platform designed could follow an open source model, allowing for open collaboration in design, development and distribution.

The immediate next step in this research would be to consider further research into the design of the platform for the content created. This research would require a more user centred approach due to the need for user interface and experience design considerations for the application developed. This platform could be further considered from an economic and consumer centred perspective, researching platform economics in this digital age to inform how this platform could support itself and the creators that create content for it. Another possible research pathway would be to consider more data and visualisation. This was addressed as part of the reflections into the design scenarios in Chapter 4. Exploring this in more detail and considering the data of a location as part of the context of that location could have interesting implications for the creation of both Digital Street Art and any platform that hosts it. As noted in the literature review, Augmented Reality (AR) technology is accelerating, and a large amount of capital and resources are going towards its development. With this in mind, it is important to note that in the future Head-Mounted AR displays could reach the point of mass adoption, resulting in this being the most prevalent type of AR. When this happens, further research could be conducted into the design Digital Street Art for that hardware, as the functional creation of content will differ.

The goal of this research was to inform design practices for creating Digital
Street Art and discuss the implications for the development of a platform to host this content. These informed practices and implications make up the conclusion of this thesis. 


\section{Conclusion}


Street Art uses the street as an artistic resource, and its location is integral to its meaning (Riggle, 2010). Augmented Reality technology affords a new way of doing this, allowing digital content to use the street as an artistic resource, this results in the concept of Digital Street Art.

The concept of Digital Street Art was developed through contextual research into the fields of Augmented Reality and Street Art. This research showed the parallels between the affordances of Augmented Reality technology, the content created with it, and the content and ideologies of Street Art. Mobile AR is an accessible technology that draws strong context from physical location, allowing for digital expressions of creativity relating to those locations. AR art, though in its infancy, has been used to talk to public space, both physical and digital. It has also been used to respond to issues and be a voice for the public.

Digital Street Art therefore uses mobile AR technology to create content that is directly related to the location it is placed in. This context can be drawn from the physical features of that location, its histories, public opinion, or its social, political and environmental issues. Digital Street Art is a reclaiming of public visual space and should be available to that public, with no curational gatekeeping or barriers to entry. This allows Digital Street Art to engage with the public space and provoke responses using features such as animation, interactivity, data visualisation and threedimensional imagery.

A platform for Digital Street Art could be considered as an extension of this research. This platform would serve to make Digital Street Art as accessible as possible to both creators and consumers. It should allow for the publication of Digital Street Art at specific locations, allowing artists to design content for and with these locations as inspiration. To support the freedom of creative expression, this platform should also allow for a number of features within AR. These features include a wide range of methodologies for AR including marker tracking, planar tracking and, where possible, facial tracking. Spatial tracking is a feature still being developed but should be included as a feature once it is more accessible. The platform should allow for two dimensional and three dimensional content and be available on as many devices as possible. This platform should not be controlled or restricted by curational gatekeeping and should not be owned by a commercial entity. To this end it could be crowd funded and maintained, either as a whole platform, or by allowing creators to support themselves alongside it through the use of their own crowd funding. Open source methods could also be used to develop and publish this platform.

This research could be developed further by focusing on the design of the platform based on the implications discussed. Along with the design of this, research could be conducted into the economics of the platform, both in terms of how it would be sustainable, and in terms of supporting creators of Digital Street Art. Further research could also consider the implication of data as part of the context of a location, using this as a source of inspiration for both physical and digital Street Art. Future research could also depend on the state of Augmented Reality as a technology and what hardware is most prevalent. 
The possibilities of Augmented Reality are endless, but we are already beginning to see where it can take us. Not only does it have many practical uses, but we are beginning to see its use as a means of creative expression.

This thesis shows one exploration of this creativity, and how that can be used to reclaim the public visual space, both physically and digitally. 


\section{List of Figures}


Figure 2. Oliver, J. (n.d.). [ARtvertiser being used to replace a large-scale billboard]. Retrieved from https://theartvertiser.com/

Figure 3. Errazuriz, S. (2017). [ Jeff Koons 'Balloon Dog' Graffiti Bombed, 2017]. Retrieved fromhttps://www.instagram.com/p/BZ4Zx6pDvVv/

Figure 4. Bishop, J. (2017). [Untitled Photo showing one of the

Downstage Exhibition works]. Retrieved from https://www.behance.net/ gallery/50007711/Standing-Ovation-An-Augmented-Reality-Exhibtion

Figure 24. Heke, N. (2013). BMD for anti-shark finning, Wellington,

2013. Retrieved from https://bmdisyourfriend.com/te-papa-shark-wall/

All other figures were either photographed or generated by the author, Stefan Peacock 


\section{References}


Ajanki, A., Billinghurst, M., Gamper, H., Järvenpää, T., Kandemir, M., Kaski, S., ... Tossavainen, T. (2011). An augmented reality interface to contextual information. Virtual Reality; Godalming, Surrey, 15(2-3), 161-173. http://dx.doi.org/10.1007/s10055-010-0183-5

Apple Inc. (2018a). ARKit - Apple Developer. Retrieved 8 July 2018, from https://developer.apple.com/arkit/

Apple Inc. (2018b). Understanding World Tracking in ARKit | Apple Developer Documentation. Retrieved 30 June 2018, from https:// developer.apple.com/documentation/arkit/understanding_world_ tracking_in_arkit

Archer, B. (1995). The Nature of Research. Co-Design, 2(11), 6-13.

Azuma, R., Baillot, Y., Behringer, R., Feiner, S., Julier, S., \& Macintyre, B. (2001). Recent advances in augmented reality. Computer Graphics and Applications, IEEE, 21(6), 34-47. https://doi.org/10.1109/38.963459

Azuma, R. T. (1997). A Survey of Augmented Reality. Presence: Teleoperators Eจ Virtual Environments, 6(4), 355-385. https://doi. org/10.1162/pres.1997.6.4.355

Banksy. (n.d.). Retrieved 13 July 2018, from http://www.banksy.co.uk/ faq.asp\#
Bengtsen, P. (2015). Carelessness or curatorial chutzpah? On controversies surrounding street art in the museum. Konsthistorisk Tidskrift/Journal of Art History, 84(4), 220-233. https://doi.org/10.1080/00233609.2015.10 95797

Benjamin, W. (1935). The Work of Art in the Age of its Technological Reproducability. Gesammelte Schriften, 7.

Billinghurst, M., Kato, H., \& Poupyrev, I. (2001). The MagicBook moving seamlessly between reality and virtuality. IEEE Computer Graphics and Applications, 21(3), 6-8. https://doi.org/10.1109/38.920621

Bishop, J. (2017). Augmented Reality on Display. Victoria University of Wellington.

Burry, M. (2016, July 13). Inside broke students' flats. Retrieved 5 July 2018, from http://thewireless.co.nz/articles/inside-broke-students-flats

Campbell, F. C. and G. (2018, January 22). 100 complaints over Wellington rental prices. NZ Herald. Retrieved from https://www. nzherald.co.nz/business/news/article.cfm?__id=3\&robjectid=11979817

Campbell, M. (2018, March 28). ARKit app downloads exceed 13M in 6 months, nearly half are games, report says. Retrieved 8 July 2018, from https://appleinsider.com/articles/18/03/29/arkit-app-downloads-exceed$13 \mathrm{~m}$-in-6-months-nearly-half-are-games-report-says Campbell, S. (2016). » EYEJACK AppSutu Eat Flies. Retrieved 10 
November 2017, from http://www.sutueatsflies.com/portfolio/eyejackapp/

Cann, G. (2017, November 19). Wellington could be heading for its worst rental crisis ever, property experts warn. Retrieved 4 July 2018, from http://www.stuff.co.nz/business/property/98941556/wellington-couldbe-heading-for-its-worst-rental-crisis-ever-property-experts-warn

Cann, G. (2018a, January 29). One modest two-bed Wellington flat, thousands of eager renters. Retrieved 4 July 2018, from http://www.stuff. co.nz/business/property/100796614/one-modest-twobed-wellington-flatthousands-of-eager-renters

Cann, G. (2018b, May 24). Wellington waterfront goes straw free, now the council wants the city to follow suit. Retrieved 6 July 2018, from http://www.stuff.co.nz/national/103366146/wellington-waterfront-goesstraw-free-now-the-council-wants-the-city-to-follow-suit

Caudell, T. P., \& Mizell, D. W. (1992). Augmented reality: an application of heads-up display technology to manual manufacturing processes. In Proceedings of the Twenty-Fifth Hawaii International Conference on System Sciences (Vol. 2, pp. 659-669) ). https://doi.org/10.1109/ HICSS.1992.183317

Chow, A. R. (2017, December 22). Snapchat and Jeff Koons Collaborate on Augmented Reality Art Project. The New York Times. Retrieved from https://www.nytimes.com/2017/10/03/arts/design/snapchat-and-jeff- koons-collaborate-on-augmented-reality-art-project.html

Codrea-Rado, A. (2017, October 10). Virtual Vandalism: Jeff Koons's 'Balloon Dog' Is Graffiti-Bombed. The New York Times. Retrieved from https://www.nytimes.com/2017/10/10/arts/design/augmented-realityjeff-koons.html

Deahl, D. (2018, June 4). Apple's ARKit 2.0 supports multiplayer and introduces a new file format. Retrieved 1 July 2018, from https://www. theverge.com/2018/6/4/17418614/arkit-update-multiplayer-announcedapple-wwdc-2018

Dey, A., Billinghurst, M., Lindeman, R. W., \& Swan, J. E. I. (2018). A Systematic Review of 10 Years of Augmented Reality Usability Studies: 2005 to 2014. Frontiers in Robotics and AI, 5. https://doi.org/10.3389/ frobt.2018.00037

Dickinson, M. (2008). The Making of Space, Race and Place New York City's War on Graffiti, 1970-the Present. Critique of Anthropology: A Journal for the Critical Reconstruction of Anthropology, 28(1), 27-45. https://doi.org/10.1177/0308275X07086556

Epic Games. (2017). AR And VR For The Rest of Us. Retrieved 1 July 2018, from https://www.unrealengine.com/en-US/blog/epic-unrealengine-wwdc-2017

Ferrel, J. (1995). Urban Graffiti: Crime, Control, and Resistance. Youth שซ 
Society, 27(1), 73-92. https://doi.org/10.1177/0044118X95027001005

Frankel, L., \& Racine, M. (2010). The Complex Field of Research: for Design, through Design, and about Design, 12.

Frayling, C. (1993). Research in Art and Design. Royal College of Art Research Papers, 1(1). Retrieved from http://researchonline.rca. ac.uk/384/3/frayling_research_in_art_and_design_1993.pdf

Garbe, J. (2014). Digital Borders and the Virtual Gallery. In Augmented Reality Art (pp. 139-147). Springer, Cham. https://doi.org/10.1007/9783-319-06203-7_7

Google LLC. (2018, May 8). ARCore Overview | ARCore. Retrieved 8 July 2018, from https://developers.google.com/ar/discover/ Gwilt, I. (2014). Augmented Reality Graffiti and Street Art. In Augmented Reality Art (pp. 189-198). Springer, Cham. https://doi. org/10.1007/978-3-319-06203-7_11

Invader. (n.d.). Invader - Home. Retrieved 13 July 2018, from https:// www.space-invaders.com/home/

Krueger, M. W., Gionfriddo, T., \& Hinrichsen, K. (1985). VIDEOPLACE—an Artificial Reality. In Proceedings of the SIGCHI Conference on Human Factors in Computing Systems (pp. 35-40). New York, NY, USA: ACM. https://doi.org/10.1145/317456.317463
Langlotz, T., Grubert, J., \& Grasset, R. (2013). Augmented reality browsers: essential products or only gadgets? Communications of the ACM, 56(11), 34-36. https://doi.org/10.1145/2527190

Layar. (n.d.). Augmented Reality | Interactive Print. Retrieved 30 June 2018, from https://www.layar.com/

Lichty, P. (2014). The Aesthetics of Liminality: Augmentation as an Art Form. In Augmented Reality Art (pp. 99-125). Springer, Cham. https:// doi.org/10.1007/978-3-319-06203-7_5

Lodi, S. (2014). Spatial Narratives in Art. In Augmented Reality Art (pp. 277-294). Springer, Cham. https://doi.org/10.1007/978-3-319-062037_16

Matt, S. (2016, February 16). Wellington students 'at mercy of price gougers' in desperate hunt for flats. Retrieved 5 July 2018, from http:// www.stuff.co.nz/national/education/76889676/wellington-students-atmercy-of-price-gougers-in-desperate-hunt-for-flats

McGarrigle, C. (2014). Augmented Interventions: Re-defining Urban Interventions with AR and Open Data. In Augmented Reality Art (pp. 81-95). Springer, Cham. https://doi.org/10.1007/978-3-319-06203-7_4

Mcneilly, H. (2018, June 30). Damp and cold flats a Scarfie rite of passage or student slum? Retrieved 4 July 2018, from http://www.stuff.co.nz/ 
business/property/104841040/damp-and-cold-flats-a-scarfie-rite-ofpassage-or-student-slum

Microsoft. (2016). Microsoft HoloLens. Retrieved 30 June 2018, from https://www.microsoft.com/en-us/hololens

Milgram, P., Takemura, H., Utsumi, A., \& Kishino, F. (1995). Augmented reality: a class of displays on the reality-virtuality continuum (Vol. 2351, pp. 282-292). https://doi.org/10.1117/12.197321

MSD. (2018). Student Allowance rates - StudyLink. MSD. Retrieved from https://www.studylink.govt.nz/products/rates/student-allowance-rates. html\#null

Niantic Inc. (2016). Catch Pokémon in the Real World with Pokémon GO! Retrieved 30 June 2018, from http://www.pokemongo.com/en-au/ Obey Giant - The Art of Shepard Fairey. (2018). Retrieved 13 July 2018, from https://obeygiant.com/

Oliver, J. (n.d.). The Artvertiser. Retrieved 21 June 2018, from https:// theartvertiser.com/

Pacheco, D., Wierenga, S., Omedas, P., Oliva, L. S., Wilbricht, S., Billib, S., ... Verschure, P. F. M. J. (2015). A location-based Augmented
Reality system for the spatial interaction with historical datasets. In 2015 Digital Heritage (Vol. 1, pp. 393-396). https://doi.org/10.1109/ DigitalHeritage.2015.7413911

Plastic Pollution Coalition. (2015, November 11). The Turtle That Became the Anti-Plastic Straw Poster Child. Retrieved 7 July 2018, from http://www.plasticpollutioncoalition.org/pft/2015/10/27/the-turtlethat-became-the-anti-plastic-straw-poster-child

PTC Inc. (2018). Vuforia | Augmented Reality. Retrieved 13 July 2018, from https://www.vuforia.com/

Public Place Recycling project. (2018). Retrieved 10 July 2018, from http://wellington.govt.nz/services/environment-and-waste/rubbish-andrecycling/recycling/public-place-recycling-project

Reitmayr, G., \& Schmalstieg, D. (2003). Location Based Applications for Mobile Augmented Reality. In Proceedings of the Fourth Australasian User Interface Conference on User Interfaces 2003 - Volume 18 (pp. 65-73). Darlinghurst, Australia, Australia: Australian Computer Society, Inc. Retrieved from http://dl.acm.org/citation.cfm?id=820086.820103

Riggle, N. A. (2010). Street Art: The Transfiguration of the Commonplaces. Journal of Aesthetics and Art Criticism, 68(3), 243-257. https://doi.org/10.1111/j.1540-6245.2010.01416.x

Ross, J. I. (2016). Routledge Handbook of Graffiti and Street Art. Routledge. 
Rowe, M., \& Hutton, F. (2012). 'Is your city pretty anyway?' Perspectives on graffiti and the urban landscape. Australian $\mathcal{E}^{2}$ New Zealand Journal of Criminology, 45(1), 66-86. https://doi.org/10.1177/0004865811431327

Sarah Banet-Weiser. (2012). Branding Creativity Creative Cities, Street Art, and 'Making your Name Sing'. In Authentic: The Politics of Ambivalence in a Brand Culture. NYU Press. Retrieved from http://www. jstor.org/stable/j.ctt9qfmw0.7

Sheavly, S. B., \& Register, K. M. (2007). Marine Debris \& Plastics: Environmental Concerns, Sources, Impacts and Solutions. Journal of Polymers and the Environment, 15(4), 301-305. https://doi.org/10.1007/ s10924-007-0074-3

Skwarek, M. (2011). AR Occupy Wall Street. Retrieved 1 July 2018, from https://aroccupywallstreet.wordpress.com

Skwarek, M. (2014). Augmented Reality Activism. In Augmented Reality Art (pp. 3-29). Springer, Cham. https://doi.org/10.1007/978-3-31906203-7_1

Sustainable Coastlines. (n.d.). Sustainable Coastlines :: Home. Retrieved 6 July 2018, from http://sustainablecoastlines.org/

Sutherland, I. E. (1968). A Head-mounted Three Dimensional Display. In Proceedings of the December 9-11, 1968, Fall Joint Computer
Conference, Part I (pp. 757-764). New York, NY, USA: ACM. https:// doi.org/10.1145/1476589.1476686

Thompson, R. C., Moore, C. J., Saal, F. S. vom, \& Swan, S. H. (2009). Plastics, the environment and human health: current consensus and future trends. Philosophical Transactions of the Royal Society of London B: Biological Sciences, 364(1526), 2153-2166. https://doi.org/10.1098/ rstb.2009.0053

Unity Technologies. (2018a). Unity - Manual: What is a Particle System? Retrieved 7 July 2018, from https://docs.unity3d.com/Manual/

PartSysWhatIs.html

Unity Technologies. (2018b). Unity for Mobile AR. Retrieved 1 July 2018, from https://unity.com/solutions/mobile-ar

Valkenburg, R., \& Dorst, K. (1998). The reflective practice of design teams. Design Studies, 19(3), 249-271. https://doi.org/10.1016/S0142$694 \mathrm{X}(98) 00011-8$

Veenhof, S., \& Skwarek, M. (2010). Augmented Reality art exhibition MoMA NYC. Retrieved 1 July 2018, from http://www.sndrv.nl/moma/

WallaMe. (2017). Augmented Reality | Hide messages in the real world. Retrieved 30 June 2018, from http://walla.me/ 
Wellington City Council. (2018, April 24). Final straw for plastic straws in the capital. Retrieved 6 July 2018, from http://wellington.govt.nz/your-

council/news/2018/04/plastic-straws 
